JOURNAL OF THE AMERICAN MATHEMATICAL SOCIETY

Volume 20, Number 4, October 2007, Pages 1003-1051

S 0894-0347(07)00561-9

Article electronically published on March 14, 2007

\title{
FINITE GROUP EXTENSIONS AND THE ATIYAH CONJECTURE
}

\author{
PETER LINNELL AND THOMAS SCHICK
}

\section{INTRODUCTION}

In 1976, Atiyah 2] constructed the $L^{2}$-Betti numbers of a compact Riemannian manifold. They are defined in terms of the spectrum of the Laplace operator on the universal covering of $M$. By Atiyah's $L^{2}$-index theorem [2, they can be used e.g. to compute the Euler characteristic of $M$.

Dodziuk 10 proved an $L^{2}$-Hodge de Rham theorem which gives a combinatorial interpretation of the $L^{2}$-Betti numbers, now in terms of the spectrum of the combinatorial Laplacian. This also generalized the definition of $L^{2}$-Betti numbers to arbitrary finite $\mathrm{CW}$-complexes. The combinatorial Laplacian can be considered to be a matrix over the integral group ring of the fundamental group $G$ of $M$. These $L^{2}$-invariants have been successfully used to give new results in differential geometry, topology, and algebra. For example, one can prove certain cases of the Hopf conjecture about the sign of the Euler characteristic of negatively curved manifolds [17, or, in a completely different direction, certain cases of the zero divisor conjecture, which asserts that $\mathbb{Q}[G]$ has no non-trivial zero divisors if $G$ is torsion-free, following from the Atiyah conjecture about $L^{2}$-Betti numbers. This paper is concerned with this algebraic aspect of the theory of $L^{2}$-Betti numbers. The methods employed will also be algebraic.

The Atiyah conjecture is the central theme of this paper. It is a prediction of the possible values of the $L^{2}$-Betti numbers mentioned above. We start with an algebraic formulation of its statement.

1.1. Definition. For a discrete group $G$, set

$$
\operatorname{lcm}(G):=\text { least common multiple of }\{|F| \mid F \leq G \text { and }|F|<\infty\},
$$

where we adopt the convention that the least common multiple of an unbounded set is $\infty$.

If $\operatorname{lcm}(G)<\infty$, the strong Atiyah conjecture over $K G$ says

$$
\operatorname{lcm}(G) \cdot \operatorname{dim}_{G}(\operatorname{ker} A) \in \mathbb{Z} \quad \forall A \in M_{n}(K G) ;
$$

in this paper $K \subset \mathbb{C}$ will always be a subring of the complex numbers which is closed under complex conjugation. We have used the adjective "strong" so as to distinguish it from the conjecture that Atiyah made in [2], which in the case $K=\mathbb{Q}$ is equivalent to $\operatorname{dim}_{G}(\operatorname{ker} A) \in \mathbb{Q}$ for all $A \in M_{n}(\mathbb{Q} G)$.

Received by the editors May 31, 2005.

2000 Mathematics Subject Classification. Primary 55N25, 16S34, 57M25.

The first author was partially supported by SFB 478, Münster.

Research of the second author was funded by DAAD (German Academic Exchange Agency).

(C)2007 American Mathematical Society 1003

Reverts to public domain 28 years from publication 
Here $A$ is considered as a bounded operator $A: l^{2}(G)^{n} \rightarrow l^{2}(G)^{n}$ (acting by the convolution product). Let $\operatorname{pr}_{\operatorname{ker} A}$ be the projection onto ker $A$. Then

$$
\operatorname{dim}_{G}(\operatorname{ker} A):=\operatorname{tr}_{G}\left(\operatorname{pr}_{\operatorname{ker} A}\right):=\sum_{i=1}^{n}\left\langle\operatorname{pr}_{\operatorname{ker} A} e_{i}, e_{i}\right\rangle_{l^{2}(G)^{n}},
$$

where $e_{i} \in l^{2}(G)^{n}$ is the vector with the identity element of $G \subset l^{2}(G)$ at the $i$ th position and zeros elsewhere.

The ring $K G$ embeds into the group von Neumann algebra $\mathcal{N} G$, and this into von Neumann's ring of affiliated operators $\mathcal{U} G$. We denote by $D G$ the division closure of $K G$ in $\mathcal{U} G$, and we let $\Sigma(G)$ be the set of all matrices over $K G$ which become invertible in $D G$. For more details about all these objects compare e.g. 44] or [36, Section 8].

1.2. Remark. By [38, Lemma 2.2] the strong Atiyah conjecture as stated in Definition 1.1 for $K=\mathbb{Q}$ is equivalent to the statement that for every finite CW-complex $X$ with fundamental group $G$, the $L^{2}$-Betti numbers fulfill $\operatorname{lcm}(G) b_{(2)}^{p}(X) \in \mathbb{Z}$.

1.3. Remark. There were more general versions of the Atiyah conjecture for groups with $\operatorname{lcm}(G)=\infty$. The geometric version then says that at least all relevant $L^{2}$ Betti numbers should be contained in the additive subgroup of $\mathbb{Q}$ generated by the numbers $|F|^{-1}, F \leq G$ with $|F|<\infty$.

However, this conjecture is false, as was shown by counterexamples in [16 and 9].

1.4. Remark. If $G$ is torsion-free, the strong Atiyah conjecture for $K G$ is particularly interesting: it implies that $K G$ is a domain, i.e. has no non-trivial zero-divisors [38. Lemma 2.4]. More precisely, the ring $D G$ which contains $K G$ is a skew field (compare e.g. [47, Lemma 3]).

There are of course also purely algebraic methods available to attack the abovementioned zero-divisor problem (and generalizations thereof). We will also give partial results in such a more general setting. More precisely:

1.5. Definition. Let $R$ be a division ring and $G$ a group. A crossed product $R * G$ is an associative ring which has as an underlying set the free $R$-module $\bigoplus_{g \in G} R \cdot \bar{g}$ with basis $\{\bar{g} \mid g \in G\}$. The multiplication is determined by set maps $\phi: G \times G \rightarrow R \backslash\{0\}$, $\sigma: G \rightarrow \operatorname{Aut}(R)$ satisfying certain conditions, and is given by $\overline{g_{1}} \cdot \overline{g_{2}}=\phi\left(g_{1}, g_{2}\right) \overline{g_{1} g_{2}}$, $\bar{g} \cdot r=(\sigma(g) r) \cdot \bar{g}$; see [43, p. 2] for further information.

We will be interested in the question of when such crossed products are domains, and in particular when they admit embeddings into skew fields.

We will throughout the paper only deal with groups $G$ with $\operatorname{lcm}(G)<\infty$. Note that $G$ is torsion-free if and only if $\operatorname{lcm}(G)=1$.

The strong Atiyah conjecture is true for elementary amenable groups, and more generally for free groups and their elementary amenable extensions [35]. Note that this class of groups is closed under finite extensions.

The strong Atiyah conjecture is also known for $\mathbb{Q} G$ if $G$ is a residually torsion-free elementary amenable group (i.e. the case $K=\mathbb{Q}$ ) 4 47, Corollary 4]. A generalization to $K=\overline{\mathbb{Q}}$, the field of algebraic numbers over $\mathbb{Q}$ in $\mathbb{C}$, is given in [11. This applies e.g. for pure braid groups and certain positive one-relator groups, but in these cases with known methods no general statement about finite extensions can be made. 
Observe that the last statement applies in particular to Artin's pure braid groups $P_{n}$, which are residually torsion-free nilpotent [14, Theorem 2.6]. Our work was motivated by the question of whether the full braid groups $B_{n}$, which are finite torsion-free extensions of $P_{n}$, also satisfy the strong Atiyah conjecture. It was also motivated by the result of Rolfsen and Zhu [45, Theorem 1] where using the property that $B_{n}$ is left orderable [15], they proved that $\mathbb{C} B_{n}$ has no non-trivial zero divisors.

In this paper, we establish this and much more general results.

The basic idea is: it is hard to deal with extensions with finite quotient $F$, but easy to handle torsion-free elementary amenable quotients. We recall in Section 2 (see Corollary 2.7 for a more general statement):

1.6. Theorem. Let $H$ be a torsion-free group which fulfills the strong Atiyah conjecture over $K H$. Suppose we have an exact sequence $1 \rightarrow H \rightarrow G \rightarrow A \rightarrow 1$, where $A$ is torsion-free and elementary amenable. Then the strong Atiyah conjecture for $K G$ is true.

For a finite extension $1 \rightarrow H \rightarrow G \rightarrow F \rightarrow 1$, our goal therefore is to find a factorization $G \stackrel{\pi}{\rightarrow} A \rightarrow F$, where $A$ is elementary amenable and torsion-free, and apply Theorem 1.6 to the extension $1 \rightarrow \operatorname{ker} \pi \rightarrow G \rightarrow A \rightarrow 1$.

One can further reduce to the case where $F$ is a finite $p$-group for a prime number $p$. So we have to answer the question: given a torsion-free group $H$ which fulfills the strong Atiyah conjecture, under which conditions on $H$ does every extension $1 \rightarrow H \rightarrow G \rightarrow F \rightarrow 1$, where $F$ is a finite $p$-group and $G$ is torsion-free, admit a factorization $G \rightarrow A \rightarrow F$ with $A$ torsion-free and elementary amenable.

We give such a criterion in Section 4. In particular we prove

1.7. Theorem. Assume that $H$ has a finite classifying space and that for each prime number $p$, the canonical map

$$
H^{*}\left(\hat{H}^{p}, \mathbb{Z} / p\right) \rightarrow H^{*}(H, \mathbb{Z} / p)
$$

from the continuous cohomology of the pro-p completion of $H$ to the cohomology of $H$ is an isomorphism. Suppose infinitely many quotients of the derived series of $H$ or of the lower central series of $H$ are torsion-free. Then the above factorization property is fulfilled.

The proof uses the pro- $p$ completions as an intermediate step to show that if no such torsion-free quotient exists, then the projection $G \rightarrow F$ induces a split injective map in cohomology (we might have to replace $F$ with a non-trivial subgroup first). Using an Atiyah-Hirzebruch spectral sequence argument, we show that the same is true for stable cohomotopy. But then a fixed-point theorem of Jackowski 25] implies that the map $G \rightarrow F$ itself splits, which contradicts the assumption that $G$ is torsion-free.

All of this can be generalized to extensions $G$ which contain torsion. Then the requirement is to find a factorization through an elementary amenable group $A$ which has as little torsion as possible. The theorem extends to this case, and moreover, the assumption on the torsion-free quotients can be somewhat relaxed.

The cohomological condition is rather strong. However, we show in Section 5 that all conditions are satisfied by Artin's pure braid group. More generally, consider the following classes of groups: finitely generated free groups, surface groups, primitive one-relator groups, knot groups and primitive link groups. Using 
these building blocks, construct $H$ as an iterated semi-direct product, such that the quotient always acts trivially on the abelianization of the kernel. Then $H$ fulfills the conditions of Theorem [1.6. By [14] the pure braid groups can be constructed in this way, and therefore the theorem can be applied to them.

We remark that, in the course of the proof, we construct torsion-free non-trivial elementary amenable quotients of the torsion-free extensions of our class of groups. This applies in particular to the full braid groups $B_{n}$, thus answering question B6 of http://grouptheory.info/ for all $n \geq 1$.

Explicitly (compare Corollary 5.41) we have

1.8. Theorem. Let $B_{n}$ be the nth classical full Artin braid group. Then the strong Atiyah conjecture for $\overline{\mathbb{Q}} B_{n}$ is true, where $\overline{\mathbb{Q}}$ is the field of algebraic numbers in $\mathbb{C}$. Moreover, $B_{n}$ is residually torsion-free nilpotent-by-finite.

The algebraic methods of this paper can also be applied to the Baum-Connes conjecture, to establish this conjecture in more new cases. This is discussed in [6], where for example the Baum-Connes conjecture is proved for the full braid groups.

\section{Atiyah CONJECTURE AND EXtension WITH AN ELEMENTARY AMENABLE QUOTIENT}

In this section, we recall some general results about the Atiyah conjecture, proved essentially in [36, 35. We will frequently make use of results in [36, 35. There, these results are stated only for $K=\mathbb{C}$. However, everything generalizes immediately to the more general rings $K$ we are considering.

2.1. Notation. We let $\mathcal{A}$ denote the class of finitely generated abelian-by-finite groups. If $\mathcal{X}$ and $\mathcal{Y}$ are classes of groups, then $G \in L \mathcal{X}$ means that every finitely generated subgroup of $G$ is contained in an $\mathcal{X}$-group, and $G \in \mathcal{X} \mathcal{Y}$ means that $G$ has a normal $\mathcal{X}$-subgroup $H$ such that $G / H$ is a $\mathcal{Y}$-group.

A group is called elementary amenable, if it belongs to the smallest class of groups which contains all solvable groups and all finite groups, and which is closed under extensions and under directed unions.

Also $\mathbb{N}$ will denote the positive integers (so 0 is excluded), and $\mathbb{N}_{0}:=\mathbb{N} \cup\{0\}$.

2.2. Proposition. Assume that $G$ is a discrete group with $\operatorname{lcm}(G)<\infty$ and $D G$, the division closure of $K G$ in $\mathcal{U} G$, is Artinian.

Then DG is semi-simple Artinian. Moreover, if $L$ is a positive integer, then

$$
L \cdot \operatorname{dim}_{G}(\operatorname{ker} A) \in \mathbb{Z} \quad \text { for each } A \in M_{n}(K G)
$$

is equivalent to

$$
L \cdot \operatorname{tr}_{G}(e) \in \mathbb{Z} \quad \text { for each projection } e \in D G .
$$

In particular, $G$ fulfills the strong Atiyah conjecture if and only if for each projection $e \in D G$ we have $\operatorname{lcm}(G) \cdot \operatorname{tr}_{G}(e) \in \mathbb{Z}$.

Proof. Semi-simplicity follows from [36, Lemma 9.4].

The remaining statements are also standard. The strong Atiyah conjecture implies the statement about the trace of the projections in $D G$ using Cramer's rule [8, Proposition 7.1.3] as in the proof of [35, Lemma 3.7].

The converse follows by noting that the semi-simple Artinian subring $D G$ of $\mathcal{U} G$ is $*$-regular, and hence for $A \in M_{n}(K G) \subset M_{n}(D G)$ we have $A M_{n}(D G)=$ 
$e M_{n}(D G)$ for a unique projection $e \in M_{n}(D G)$. Thus the $G$-dimension of the range of $A$ is equal to the $G$-trace of the projection $e$, the $G$-dimension of the kernel of $A$ is equal to the $G$-trace of the projection $1-e$, and we can apply [36. Corollary 13.11] to see that $L \operatorname{tr}_{G}(e) \in \mathbb{Z}$.

2.3. Lemma. Assume that $H$ is a discrete group, $D H$ is Artinian and there is a positive integer $L$ such that

$$
L \cdot \operatorname{tr}_{H}(e) \in \mathbb{Z} \quad \text { if } e \in D H \text { is a projection. }
$$

Suppose $H \leq G$ and the index $[G: H]<\infty$. Then $D G$ is semi-simple Artinian and

$$
[G: H] \cdot L \cdot \operatorname{tr}_{G}(e) \in \mathbb{Z} \quad \text { if } e \in D G \text { is a projection. }
$$

Proof. This follows immediately from [36, Lemma 9.4], Proposition 2.2 and [47, Proposition 4]. Alternatively, a direct argument is given in [36, Proof of Lemma 13.12].

2.4. Lemma. Assume that $H$ is a group with $\operatorname{lcm}(H)<\infty$. Let $G$ be a finite extension of $H$ with quotient group $G / H$ and projection $\pi: G \rightarrow G / H$.

If there is $L \in \mathbb{N}$ such that for the inverse images $H_{p} \leq G$ of all Sylow subgroups of $G / H$

$$
L \cdot \operatorname{lcm}\left(H_{p}\right) \cdot \operatorname{dim}_{H_{p}}(\operatorname{ker} A) \in \mathbb{Z} \quad \forall A \in M_{n}\left(K H_{p}\right),
$$

then

$$
L \cdot \operatorname{lcm}(G) \cdot \operatorname{dim}_{G}(\operatorname{ker} A) \in \mathbb{Z} \quad \forall A \in M_{n}(K G) .
$$

In particular, the strong Atiyah conjecture for all the $H_{p}$ implies the strong Atiyah conjecture for $G$.

Proof. Write $|G / H|=p_{1}^{n_{1}} \ldots p_{k}^{n_{k}}$ where the $p_{j}$ are different primes. Let $H_{p_{j}}$ be the inverse image under $\pi$ of a $p_{j}$-Sylow subgroup of $G / H$. This implies that $p_{j}$ does not divide $\left[G: H_{p_{j}}\right]=|G / H| / p_{j}^{n_{j}}$, i.e. the greatest common divisor of all $\left[G: H_{p_{j}}\right]$ is one.

For trivial reasons we have the following divisibility relation:

$$
\operatorname{lcm}\left(H_{p_{j}}\right) \mid \operatorname{lcm}(G) \quad \forall j .
$$

If $A \in M_{n}(K G)$, then, by assumption and by [47, Proposition 4],

$$
\begin{array}{ll} 
& L \cdot \operatorname{lcm}\left(H_{p_{j}}\right) \cdot\left[G: H_{p_{j}}\right] \cdot \operatorname{dim}_{G}(\operatorname{ker}(A)) \in \mathbb{Z}, \\
\text { consequently } & L \cdot\left[G: H_{p_{j}}\right] \cdot \operatorname{lcm}(G) \cdot \operatorname{dim}_{G}(\operatorname{ker}(A)) \in \mathbb{Z} .
\end{array}
$$

Passing to the greatest common divisor we get

$$
L \cdot \operatorname{lcm}(G) \cdot \operatorname{dim}_{G}(\operatorname{ker} A)=\text { g.c.d. }\left\{\left[G: H_{p_{j}}\right]\right\} \cdot L \cdot \operatorname{lcm}(G) \cdot \operatorname{dim}_{G}(\operatorname{ker} A) \in \mathbb{Z} .
$$

The following lemma is needed to arrive at the purely algebraic results - beyond the Atiyah conjecture - we want to obtain. Information on Ore domains can be found in [36, §4].

2.5. Lemma. Let $G$ be an elementary amenable group, let $k$ be an Ore domain, and let $k * G$ be a crossed product. If $k * F$ is a domain for all finite subgroups $F$ of $G$, then $k * G$ is an Ore domain, consequently it has a right quotient ring which is a skew field. 
Proof. First suppose $G$ is finitely generated abelian-by-finite. If $k$ is a skew field, this is the statement of [36, Corollary 4.5]; it also follows from [29, Lemma 4.1]. For an arbitrary Ore domain $k$, let $S=k \backslash\{0\}$. Then $k S^{-1}$ is a skew field and $k S^{-1} * H \cong(k * H) S^{-1}$ for all subgroups $H$ of $G$. From the first sentence, $k S^{-1} * G$ is an Ore domain, and we deduce that $k * G$ is an Ore domain.

The general case is proved by transfinite induction. Let $\mathcal{A}$ be the class of all finitely generated abelian-by-finite groups. Let $\mathcal{Y}_{0}$ denote the class of finite groups, and define inductively $\mathcal{Y}_{\alpha}:=\left(L \mathcal{Y}_{\alpha-1}\right) \mathcal{A}$ if $\alpha$ is a successor ordinal, and $\mathcal{Y}_{\alpha}:=$ $\bigcup_{\beta<\alpha} \mathcal{Y}_{\beta}$ if $\alpha$ is a limit ordinal (cf. [35, Lemma 4.9]). Then, by the proof of [35, Lemma 4.9(ii)], the class of elementary amenable groups is $\bigcup_{\alpha \geq 0} \mathcal{Y}_{\alpha}$.

Let $\alpha$ be the least ordinal such that $G \in \mathcal{Y}_{\alpha}$. Then $\alpha$ cannot be a limit ordinal, and the result is certainly true if $\alpha=0$. Therefore, we may write $\alpha=\beta+1$, and assume that the result is true for all groups in $\mathcal{Y}_{\beta}$. If $U \in L \mathcal{Y}_{\beta}$, then since $k * E$ is an Ore domain for every finitely generated subgroup $E$ of $U$, it is clear that $k * U$ is an Ore domain. Hence, we may assume that the result is true for groups in $L \mathcal{Y}_{\beta}$. Now $G$ has a normal subgroup $D$ such that $D \in L \mathcal{Y}_{\beta}$ and $G / D \in \mathcal{A}$. Furthermore, if $E / D$ is a finite subgroup of $G / D$, then $E \in L \mathcal{Y}_{\beta}$ by the proof of [35. Lemma 4.9(iv)]. The result follows from the case first dealt with, where we replace $G$ with $G / D$ and $k$ with $k * D$.

2.6. Proposition. Let $1 \rightarrow H \rightarrow G \rightarrow A \rightarrow 1$ be an exact sequence of groups. Fix $K=\bar{K} \subset \mathbb{C}$ and $L \in \mathbb{N}$. Suppose $A$ is elementary amenable and $D H$ is Artinian. For a subgroup $E \leq A$, let $H_{E}$ denote the inverse image of $E$ in $G$.

(i) Assume that we can find $L \in \mathbb{N}$ such that for all finite subgroups $E \leq A$,

$$
L \cdot \operatorname{tr}_{H_{E}}(e) \in \mathbb{Z} \quad \text { for each projection } e \in D H_{E} .
$$

Then $D G$ is semi-simple Artinian and

$$
L \cdot \operatorname{tr}_{G}(e) \in \mathbb{Z} \quad \text { for each projection } e \in D G .
$$

(ii) With the setup in (i), assume further that the identity map on KH induces an isomorphism

$$
K H_{\Sigma(H)} \rightarrow D H, \quad \text { where } \Sigma(H) \text { is defined in Definition [1.1] }
$$

Then the identity map on $K G$ induces an isomorphism $K G_{\Sigma(G)} \rightarrow D G$.

(iii) Let $k$ be a domain and $k * G$ be a crossed product. Assume that $k * H$ embeds into a skew field $D_{H}$ and the twisted action of $A$ on $k * H$ extends to a twisted action on $D_{H}$ (in particular, we can form $D_{H} * A$ ). Assume that $D_{H} * E$ is a domain for all finite subgroups $E$ of $A$. Then $k * G$ can be embedded in a skew field.

Proof. We shall prove parts (i) and (ii) by the standard transfinite induction argument as used in Lemma 2.5, and we shall adopt the same notation as defined in the second paragraph there. The proof of (i) is similar to [47, Proposition 8]. Thus we have two cases to consider:

(1) $A$ has a normal subgroup $B$ such that $A / B \in \mathcal{A}$ and the result is known with $B$ in place of $A$. Then in (i) we use 36, Lemma 13.10, parts (i) and (iii)], while in (ii) we use [36, Lemma 13.10(ii)], noting that $\mathbb{C}$ can be replaced by any subfield of $\mathbb{C}$ which is closed under complex conjugation. 
(2) $A$ is the directed union of groups $A_{i}$, and the result is known with $A_{i}$ in place of $A$ for all $i$. In (i) we apply [36, Lemma 13.5, parts (i) and (ii)], while in (ii) we apply [36, Lemma 13.5(iii)]. Again we note that $\mathbb{C}$ can be replaced by any subfield of $\mathbb{C}$ which is closed under complex conjugation.

For the proof of part (iii) of the proposition, observe that $(k * H) * G / H=k * G$. By Lemma 2.5 the ring $D_{H} * A$, and hence also $(k * H) * A=k * G$, embeds into a skew field.

2.7. Corollary. Under the assumptions of Proposition 2.6, if $L=\operatorname{lcm}(G)$, then the strong Atiyah conjecture is true for $K G$.

If, for every finite subgroup $E$ of $A, K H_{E}$ fulfills the strong Atiyah conjecture, then we may take $L=\operatorname{lcm}(G)$. Hence under this condition the strong Atiyah conjecture for $K G$ holds.

Proof. This follows immediately from Proposition 2.6 and Proposition 2.2 .

2.8. Corollary. If $G$ is elementary amenable and $\operatorname{lcm}(G)<\infty$, then the strong Atiyah conjecture holds for $\mathbb{C} G$.

Proof. This follows since the strong Atiyah conjecture is true for $\mathbb{C} G$ if $G$ is a trivial group.

\section{More positive Results about the Atiyah Conjecture}

In this section, we recall a few more known results about the Atiyah conjecture, proved in particular in [47] and [11].

3.1. Definition. Let $\mathcal{D}$ be the smallest non-empty class of groups such that:

(1) If $G$ is torsion-free and $A$ is elementary amenable, and we have a projection $p: G \rightarrow A$ such that $p^{-1}(E) \in \mathcal{D}$ for every finite subgroup $E$ of $A$, then $G \in \mathcal{D}$.

(2) $\mathcal{D}$ is subgroup closed.

(3) Let $G_{i} \in \mathcal{D}$ be a directed system of groups with homomorphisms $\phi_{i j}: G_{i} \rightarrow$ $G_{j}$ or $\phi_{i j}: G_{j} \rightarrow G_{i}$, respectively, for $i<j$, and assume that $G$ is its direct limit or inverse limit, respectively. Then $G \in \mathcal{D}$.

3.2. Theorem ([48, Theorem 1], [11, Theorem 1.4]). Assume that $\overline{\mathbb{Q}}$ is the algebraic closure of $\mathbb{Q}$ in $\mathbb{C}$ and $G \in \mathcal{D}$. Then the strong Atiyah conjecture is true for $\overline{\mathbb{Q}} G$.

3.3. Corollary. The strong Atiyah conjecture is true for $\overline{\mathbb{Q}} G$, if $G$ is residually torsion-free nilpotent or if $G$ is residually torsion-free solvable.

\section{Finite extensions and the Atiyah conjecture}

In this section, we give an abstract criterion when the strong Atiyah conjecture for a torsion-free group $G$ implies the strong Atiyah conjecture for all of its extensions with finite quotient, and more generally for all extensions with elementary amenable quotient.

The strategy is the following: making use of Lemma 2.4 instead of investigating all of the finite extensions at once, we study them one prime at a time (concentrating on those extensions where the quotient is a finite $p$-group).

The crucial condition is cohomological completeness, defined in Definition 4.3 . This is expressed in terms of pro- $p$ completions and their continuous cohomology, following the definitions and notation of [50]. 
Our condition is used to show that each finite extension $G$ of the given group $H$ is an extension of a suitable subgroup of $H$ with elementary amenable quotient $A$, such that $A$ contains as little torsion as theoretically possible. This allows us to apply standard results to conclude the Atiyah conjecture for $G$.

Before we obtain these results, we start with a comparably easy other condition, which is also of cohomological type.

\subsection{Groups with Euler characteristic equal to one.}

4.1. Theorem. Assume that $H$ is a group with finite classifying space $B H$, and with Euler characteristic

$$
\chi(H)=\chi(B H) \in\{1,-1\} .
$$

Suppose the strong Atiyah conjecture is true for $K H$ and we have an exact sequence

$$
1 \rightarrow H \rightarrow G \rightarrow Q \rightarrow 1,
$$

such that $Q$ is elementary amenable and $\operatorname{lcm}(G)<\infty$.

Then the strong Atiyah conjecture is true for $K G$.

Proof. Since $H$ has a finite classifying space, it is torsion-free. Because of Proposition 2.6. Corollary 2.7 and Lemma 2.4, it is sufficient to assume that $Q$ is a finite $p$-group.

In this situation, we will prove that $|Q|$ divides $\operatorname{lcm}(G)$. It then follows from Lemma 2.3 that $K G$ fulfills the strong Atiyah conjecture.

Since the Euler characteristic is multiplicative [7, Proposition 7.3], $\chi(G)= \pm \frac{1}{|Q|}$. By [7, Theorem 9.3], $\operatorname{lcm}(G) \cdot \chi(G) \in \mathbb{Z}$, therefore $|Q|$ divides $\operatorname{lcm}(G)$. This concludes the proof.

This result is less interesting than it might seem to be because a non-trivial finite extension of a group $H$ with $\chi(H)= \pm 1$ cannot be torsion-free (which is well known and follows from the argument in the proof of Theorem 4.1).

\subsection{Cohomology of groups and their completions.}

4.2. Definition. For a discrete group $G$ and a prime number $p$, let $\hat{G}^{p}$ denote the pro-p completion of $G$. This is the inverse limit of the system of finite $p$-group quotients of $G$. Then $\hat{G}^{p}$ is a compact totally disconnected group, and we have a (not necessarily injective) canonical homomorphism $G \rightarrow \hat{G}^{p}$. The cohomology of a profinite group like $\hat{G}^{p}$ in this paper will always be its Galois cohomology [50] (sometimes also called continuous cohomology).

4.3. Definition. A discrete group $G$ is called cohomologically complete if for each prime number $p$ the homomorphism

$$
H^{*}\left(\hat{G}^{p}, \mathbb{Z} / p\right) \rightarrow H^{*}(G, \mathbb{Z} / p)
$$

is an isomorphism. Here the action on $\mathbb{Z} / p$ is assumed to be trivial. Define cohomological $p$-completeness by requiring the same only for $p$.

4.5. Lemma. Let $G$ be any group. Then in low degrees we get isomorphisms

$$
\begin{aligned}
& H^{0}\left(\hat{G}^{p}, \mathbb{Z} / p\right) \stackrel{\cong}{\leftrightarrows} H^{0}(G, \mathbb{Z} / p), \\
& H^{1}\left(\hat{G}^{p}, \mathbb{Z} / p\right) \stackrel{\cong}{\leftrightarrows} H^{1}(G, \mathbb{Z} / p),
\end{aligned}
$$


and an injection

$$
H^{2}\left(\hat{G}^{p}, \mathbb{Z} / p\right) \hookrightarrow H^{2}(G, \mathbb{Z} / p)
$$

Proof. This is well known; for the convenience of the reader we give an argument. The statement for $H^{0}$ is obvious from the definition. Next, assume $V$ is a normal subgroup of $G$ such that $G / V$ is a finite $p$-group. Then we get the following exact sequence in low cohomology degrees:

$$
\begin{aligned}
0 \rightarrow H^{1}(G / V, \mathbb{Z} / p) \rightarrow H^{1}(G & , \mathbb{Z} / p) \\
& \rightarrow H^{1}(V, \mathbb{Z} / p)^{G} \rightarrow H^{2}(G / V, \mathbb{Z} / p) \rightarrow H^{2}(G, \mathbb{Z} / p) .
\end{aligned}
$$

Passing to the direct limit, as $V$ varies over all normal subgroups of $p$-power index, we obtain the following exact sequence (since taking direct limits is exact):

$$
\begin{aligned}
0 \rightarrow H^{1}\left(\hat{G}^{p}, \mathbb{Z} / p\right) \rightarrow H^{1}(G & , \mathbb{Z} / p) \\
& \rightarrow \lim _{V} H^{1}(V, \mathbb{Z} / p)^{G} \rightarrow H^{2}\left(\hat{G}^{p}, \mathbb{Z} / p\right) \rightarrow H^{2}(G, \mathbb{Z} / p) .
\end{aligned}
$$

To prove the remaining statements, we only have to prove that the limit in the middle is zero. Now, given any such $V$, an element $x \in H^{1}(V, \mathbb{Z} / p)$ is a homomorphism $x: V \rightarrow \mathbb{Z} / p$. By Lemma 4.11, the kernel of $x$ (which has index 1 or $p$ in $V$ ) contains a subgroup $W$ which is normal in $G$, and such that $V / W$ is a finite $p$-group. Then also $G / W$ is a finite $p$-group, and by construction of $W$ the image of $x$ (which is just the restriction of the homomorphism) in $H^{1}(W, \mathbb{Z} / p)$, and therefore also in the limit, is zero. Since $V$ and $x$ were arbitrary, $\lim H^{1}(V, \mathbb{Z} / p)=0$, as desired.

4.6. Example. Let $F$ be an arbitrary free group (not necessarily finitely generated). Then $F$ is cohomologically complete.

Proof. The case where $F$ is finitely generated is contained in Example [5.29] and Proposition 5.30. The general case is proved in [30], just observing that $F$ and $\hat{F}^{p}$ have cohomological dimension 1 and that (4.4) is always an isomorphism in degrees 0 and 1.

4.7. Lemma. Assume that $G$ is cohomologically $p$-complete and $A$ is a finite discrete p-primary $\hat{G}^{p}$-module. Then the homomorphism $H^{*}\left(\hat{G}^{p}, A\right) \rightarrow H^{*}(G, A)$ is an isomorphism.

Assume that $\mathfrak{Q}$ is a profinite group such that $H^{k}(\mathfrak{Q}, \mathbb{Z} / p)$ is finite for every $k$. If $A$ is a discrete finite p-primary $\mathfrak{Q}$-module, then $H^{k}(\mathfrak{Q}, A)$ is finite for every $k \in \mathbb{N}_{0}$.

Proof. Because of the corollary to [50, Proposition 20] $A$ has a composition series whose successive quotients are isomorphic to $\mathbb{Z} / p$. We can now use induction on the length of this composition series and the five lemma applied to the long exact cohomology sequences associated to short exact sequences of coefficients (on the discrete as well as on the pro- $p$ side) to conclude the proof. Existence of these long exact sequences is proved in [53, Theorem 9.3.3] and [7, Proposition 6.1]. Compatibility of the boundary maps with the comparison maps follows from the constructions.

4.8. Definition. A set of subgroups $\mathcal{U}$ of a group $G$ is cofinal among a set of subgroups $\mathcal{V}$ of $G$, if for each $V \in \mathcal{V}$ there is $U \in \mathcal{U}$ with $U \subset V$. 
4.9. Definition. The subgroups in the lower central series of a group $G$ are called $\gamma_{n}(G)$. They are inductively defined by $\gamma_{1}(G):=G$ and $\gamma_{n+1}(G):=\left[\gamma_{n}(G), G\right]$. For two subgroups $U, V$ of $G,[U, V]$ denotes the subgroup generated by commutators $[u, v]:=u^{-1} v^{-1} u v$ with $u \in U$ and $v \in V$.

The group $G$ is called nilpotent (of class $\leq n-1$ ), if $\gamma_{n}(G)=\{1\}$ for some $n \in \mathbb{N}$.

We define the derived series $G^{(n)}$ inductively by $G^{(1)}:=G$ and $G^{(n+1)}:=$ $\left[G^{(n)}, G^{(n)}\right]$. A group is called solvable (of class $\leq n-1$ ) if $G^{(n)}=\{1\}$.

The following lemma will be used in the construction of cofinal sets of subgroups at various places.

4.10. Lemma. Let $F$ be a finite group and $I$ any index set. Then the product $G:=\prod_{i \in I} F$ is locally finite and in particular, elementary amenable.

Proof. We show that every finitely generated subgroup of $G$ is finite. Let $g_{1}, \ldots, g_{n}$ with $g_{k}=\left(f_{i}^{k}\right)_{i \in I}, f_{i}^{k} \in F$, be a finite collection of elements of $G$, and let $H$ be the subset of $G$ generated by $g_{1}, \ldots, g_{n}$. Define the map $\mu: I \rightarrow F^{n}$ by $\mu(i):=$ $\left(f_{i}^{1}, \ldots, f_{i}^{n}\right)$. The target of $\mu$ is a finite set. Therefore, there is a finite subset $J \subset I$ such that for each $i \in I$ there is $j \in J$ with $\mu(i)=\mu(j)$.

Let $p: G=\prod_{i \in I} F \rightarrow \prod_{j \in J} F=: E$ be the obvious projection. Observe that $E$ is finite since $J$ is finite. We prove that the restriction of $p$ to $H$ is injective, which implies that the finitely generated subgroup $H$ of $G$ is finite.

To do this, let $x=g_{r_{1}}^{n_{1}} \cdots g_{r_{N}}^{n_{N}} \in H$ be an element of the kernel of $p$. That means that $\left(f_{j}^{r_{1}}\right)^{n_{1}} \ldots\left(f_{j}^{r_{N}}\right)^{n_{N}}=1 \in F$ for each $j \in J$. But for an arbitrary $i \in I$ we find $j \in J$ with $f_{i}^{r_{k}}=f_{j}^{r_{k}}$ for each $k=1, \ldots, N$ by our choice of $J$. In other words, $1=g_{r_{1}}^{n_{1}} \cdots g_{r_{N}}^{n_{N}}=x \in G$, i.e. the kernel of $\left.p\right|_{H}$ indeed is trivial.

4.11. Lemma. Let $\left\{U_{i}\right\}_{i \in I}$ be a set of normal subgroups of a group $G$. Define $V:=\bigcap_{i \in I} U_{i}$.

(1) If $G / U_{i}$ is torsion-free, or nilpotent of class $\leq n$, or solvable of class $\leq n$, respectively, for every $i \in I$, then $G / V$ has the same property.

(2) If $I$ is finite and $G / U_{i}$ is finite, or is a finite p-group, or is elementary amenable, respectively, for every $i \in I$, then $G / V$ has the same property.

Fix a normal subgroup $U$ of $G$ and a subset $A$ of the set of all automorphisms of G. Set $V:=\bigcap_{\alpha \in A} \alpha(U)$.

(1) If $G / U$ is torsion-free, or nilpotent, or solvable, respectively, then $G / V$ has the same property.

(2) If $A$ is finite and $G / U$ is finite, or is a finite p-group, or is elementary amenable, respectively, then $G / V$ has the same property.

(3) Assume that $G$ is finitely generated and $G / U$ is finite or is a finite p-group, respectively. Then $G / V$ is also finite or a finite p-group, respectively. In particular, $U$ contains a characteristic subgroup $W$ such that $G / W$ is finite, or a finite p-group, respectively.

(4) If $G$ is finitely generated and $G / U$ is solvable-by-finite (i.e. has a solvable subgroup of finite index), then $G / V$ is also solvable-by-finite.

Proof. Observe that $V$ is the kernel of the projection $G \rightarrow \prod_{i \in I} G / U_{i}=: P$. Consequently, $G / V$ is a subgroup of the infinite product $P$. Now $P$ is torsion-free, or nilpotent of class $\leq n$, or solvable of class $\leq n$, respectively, if and only if the same is true for every factor $G / U_{i}$. If $I$ (and therefore the product) is finite, then we 
also conclude that $P$ is finite, or a finite $p$-group, or elementary amenable, if every $G / U_{i}$ has the same property. Since all these properties are inherited by subgroups, the first part of the lemma follows.

For the second part set $I:=A$ and $U_{\alpha}:=\alpha(U)$ for $\alpha \in I$. The first two statements now follow from the first part by observing that $G / \alpha(U)$ is isomorphic to $G / U$ for every $\alpha \in A$. For the third statement observe that, since $G$ is finitely generated, for each fixed finite group $E$ there are only finitely many different homomorphisms from $G$ to $E$. Consequently, since all the groups $G / \alpha(U)$ are isomorphic finite groups, there is only a finite number of different subgroups $\alpha(U)$ (being the kernel of finitely many homomorphisms), and the statement follows from the second statement. Putting this together with the first statement yields the fourth statement.

4.12. Lemma. Let $1 \rightarrow H \rightarrow G \stackrel{\pi}{\rightarrow} Q \rightarrow 1$ be an exact sequence of discrete groups where $H=\operatorname{ker} \pi$, and let $p$ be a prime number. Then the following sequence of pro-p completions is exact:

$$
\hat{H}^{p} \rightarrow \hat{G}^{p} \stackrel{\hat{\pi}}{\longrightarrow} \hat{Q}^{p} \rightarrow 1 .
$$

The kernel of $\hat{\pi}$ is exactly the closure of $H$ in $\hat{G}^{p}$. If the subgroups $U \cap H$, where $U$ runs through all normal subgroups of $G$ with p-power index, are cofinal among all normal subgroups of $H$ with p-power index, then we get the exact sequence

$$
1 \rightarrow \hat{H}^{p} \rightarrow \hat{G}^{p} \stackrel{\hat{\pi}}{\rightarrow} \hat{Q}^{p} \rightarrow 1 .
$$

This is the case in particular if $Q$ is a finite p-group (which implies $Q=\hat{Q}^{p}$ ).

Proof. The first statement is a standard fact; compare [53, Exercise 1.6.(10)] and use the property that the image of $\hat{H}^{p}$ in $\hat{G}^{p}$ is closed, because $\hat{H}^{p}$ is compact and $\hat{G}^{p}$ is Hausdorff. The kernel of $\hat{\pi}$ is the closure in $\hat{G}^{p}$ of $H$, in other words is the completion of $H$ with respect to the system of normal subgroups $H \cap U$, where $U \triangleleft G$ and $G / U$ is a finite $p$-group. For the second statement, we therefore have to show that this completion coincides with $\hat{H}^{p}$. By [53, Exercise 1.6(4)] this is the case under the assumption that the system $H \cap U$ is cofinal among all normal subgroups of $H$ of $p$-power index.

Assume now that $Q$ is a finite $p$-group.

It remains to check that whenever $V \triangleleft H$ such that $H / V$ is a finite $p$-group, we can find $U \triangleleft G$ with $G / U$ another finite $p$-group and $U \cap H \subset V$. The intersection $W$ of all (finitely many) conjugates $V_{i}$ of $V$ in $G$ is contained in $V$ and is normal in $G$. Its index in $G$ is a power of $p$ if and only if the same is true for its index in $H$ (since $G / H$ is a finite $p$-group). The latter follows from Lemma 4.11, Therefore $W$ is the normal subgroup we had to find, and the last assertion of the lemma follows.

The following results establish cohomological $p$-completeness.

4.13. Proposition. Assume that $H$ is a discrete group which is cohomologically p-complete. Let

$$
1 \rightarrow H \rightarrow G \rightarrow Q \rightarrow 1
$$

be an exact sequence of groups, where $Q$ is a finite p-group. Then $G$ is also cohomologically p-complete. 
Proof. The exact sequence $1 \rightarrow H \rightarrow G \rightarrow Q \rightarrow 1$ induces by Lemma 4.12 an exact sequence

$$
1 \rightarrow \hat{H}^{p} \rightarrow \hat{G}^{p} \rightarrow Q \rightarrow 1 .
$$

Now we have two spectral sequences with $E_{2}^{l, q}$-terms

$$
H^{l}\left(Q, H^{q}\left(\hat{H}^{p}, \mathbb{Z} / p\right)\right) \quad \text { or } \quad H^{l}\left(Q, H^{q}(H, \mathbb{Z} / p)\right)
$$

converging to $H^{l+q}\left(\hat{G}^{p}, \mathbb{Z} / p\right)$ or $H^{l+q}(G, \mathbb{Z} / p)$, respectively. By assumption on $H$ the natural map between Galois and ordinary cohomology induces an isomorphism between the $E_{2}$-terms, and consequently the isomorphism between $H^{*}\left(\hat{G}^{p}, \mathbb{Z} / p\right)$ and $H^{*}(G, \mathbb{Z} / p)$ that we had to establish.

4.14. Definition. Let $V$ be a vector space (over any field), or let $V$ be an abelian group, and $A: V \rightarrow V$ an automorphism of $V$. We say $A$ acts unipotently on $V$, if $\left(A-\mathrm{id}_{V}\right)^{n}=0$ for some $n \in \mathbb{N}$.

If a group $G$ acts on $V$ via $\alpha: G \rightarrow \operatorname{Aut}(V)$, we call the action of $G$ unipotent if there is $m \in \mathbb{N}$ such that $\left(\alpha\left(g_{1}\right)-\mathrm{id}_{V}\right) \cdots\left(\alpha\left(g_{m}\right)-\mathrm{id}_{V}\right)=0$ for all $g_{1}, \ldots, g_{m} \in G$.

4.15. Theorem. Let $1 \rightarrow H \rightarrow G \rightarrow Q \rightarrow 1$ be an exact sequence of groups. Assume that $H$ is finitely generated, and that $H$ as well as $Q$ are cohomologically p-complete for some prime number $p$. Assume that $H^{i}(H ; \mathbb{Z} / p)$ is finite for all $i \in \mathbb{N}$ (this is true e.g. if $H$ has a finite classifying space), and that each element of $Q$ acts unipotently on $H_{1}(H ; \mathbb{Z} / p)$. Then $G$ is cohomologically p-complete.

If, moreover, $H^{k}(Q, \mathbb{Z} / p)$ is finite for each $k \in \mathbb{N}$, then $H^{k}(G, \mathbb{Z} / p)$ is finite for each $k \in \mathbb{N}$.

This theorem with proof is modeled after [49, Exercise 1) and 2) on p. 13]. In its present formulation, it was suggested to us by Guido Mislin. To prove it, we first need a sequence of auxiliary results.

4.16. Proposition. Assume that $G$ is cohomologically p-complete, and $U$ is a normal subgroup of $G$ such that $G / U$ is a finite p-group. Then $U$ is cohomologically p-complete, as well.

Proof. We have the exact sequence $1 \rightarrow U \rightarrow G \rightarrow G / U \rightarrow 1$ and, by Lemma 4.12 an exact sequence $1 \rightarrow \hat{U}^{p} \rightarrow \hat{G}^{p} \rightarrow G / U \rightarrow 1$. Since $U$ has finite index in $G$, $\operatorname{coind}_{U}^{G} \mathbb{Z} / p$ is a finite $G$-module and, therefore, it is the restriction of $\operatorname{coind}_{\tilde{U}^{p}}^{\hat{G}^{p}} \mathbb{Z} / p$ to $G$, which is itself a finite discrete $\hat{G}^{p}$-module. The discrete Shapiro isomorphism [7. Proposition 6.2]

$$
\phi: H^{k}\left(G, \operatorname{coind}_{U}^{G} \mathbb{Z} / p\right) \rightarrow H^{k}(U, \mathbb{Z} / p)
$$

is given as composition

$$
H^{k}\left(G, \operatorname{coind}_{U}^{G} \mathbb{Z} / p\right) \stackrel{i^{*}}{\longrightarrow} H^{k}\left(U, \operatorname{res}_{U} \operatorname{coind}_{U}^{G} \mathbb{Z} / p\right) \rightarrow H^{k}(U, \mathbb{Z} / p),
$$

the first map being induced from the inclusion $i: U \hookrightarrow G$, and the second induced from the coefficient homomorphism. The profinite Shapiro isomorphism [49, Proposition 10]

$$
\hat{\phi}: H^{k}\left(\hat{G}^{p}, \operatorname{coind}_{\hat{U}^{p}}^{\hat{G}^{p}} \mathbb{Z} / p\right) \rightarrow H^{k}\left(\hat{U}^{p}, \mathbb{Z} / p\right)
$$


is given in exactly the parallel way. Naturality implies that we get a commutative diagram

$$
\begin{array}{ccc}
H^{k}\left(\hat{G}^{p}, \operatorname{coind}_{\hat{U}^{p}}^{\hat{G}^{p}} \mathbb{Z} / p\right) & \stackrel{\hat{\phi}}{\longrightarrow} H^{k}\left(\hat{U}^{p}, \mathbb{Z} / p\right) \\
\downarrow \alpha_{G} & & \downarrow^{\alpha_{U}} \\
H^{k}\left(G, \operatorname{coind}_{U}^{G} \mathbb{Z} / p\right) & \stackrel{\phi}{\longrightarrow} H^{k}(U, \mathbb{Z} / p) .
\end{array}
$$

Because $G$ is cohomologically $p$-complete and $\operatorname{coind}_{\hat{U} p}^{\hat{G}^{p}} \mathbb{Z} / p$ is a finite discrete $\hat{G}^{p}$ module, by Lemma 4.7, $\alpha_{G}$ is an isomorphism. It follows that

$$
\alpha_{U}: H^{k}\left(\hat{U}^{p}, \mathbb{Z} / p\right) \rightarrow H^{k}(U, \mathbb{Z} / p)
$$

is an isomorphism, as we had to prove.

4.17. Lemma. Assume that $V$ is a $\mathbb{Z} / p$-vector space and $A: V \rightarrow V$ is a unipotent automorphism of $V$, i.e. $\left(A-\mathrm{id}_{V}\right)^{n}=0$ for some $n \geq 0$. Then, $A^{p^{k}}=\mathrm{id}_{V}$ if $p^{k} \geq n$.

Proof. If $p^{k} \geq n$, then

$$
0=\left(A-\mathrm{id}_{V}\right)^{p^{k}}=\sum_{j=0}^{p^{k}}\left(\begin{array}{c}
p^{k} \\
j
\end{array}\right)(-1)^{j} A^{j}=\mathrm{id}_{V}-A^{p^{k}},
$$

since $\left(\begin{array}{c}p^{k} \\ j\end{array}\right)$ is divisible by $p$ for $0<j<p^{k}$, and $V$ is a $\mathbb{Z} / p$-vector space.

4.18. Definition. For a discrete group $H$, we define the $p$-lower central series inductively by $\gamma_{1}^{p}(H):=H$, and for $k>1 \gamma_{k}^{p}(H):=\left\langle\left[\gamma_{k-1}^{p}(H), H\right], \gamma_{k-1}^{p}(H)^{p}\right\rangle$, the subgroup of $H$ generated by the commutators $\left[\gamma_{k-1}^{p}(H), H\right]$ and all $p$ th powers of elements in $\gamma_{k-1}^{p}(H)$. Observe that these are characteristic subgroups. Then $\gamma_{1}^{p}(H) / \gamma_{2}^{p}(H)=H_{1}(H, \mathbb{Z} / p)$ and, more generally, there is a projection

$$
H_{1}\left(\gamma_{k-1}(H), \mathbb{Z} / p\right) \rightarrow \gamma_{k-1}^{p}(H) / \gamma_{k}^{p}(H),
$$

in particular, $\gamma_{k-1}^{p}(H) / \gamma_{k}^{p}(H)$ is a $\mathbb{Z} / p$-vector space for each $k \geq 2$.

If $H$ is a finite group of order $p^{k}$, then $\gamma_{k+1}^{p}(H)=\{1\}$. The smallest $l \in \mathbb{N}$ such that $\gamma_{l+1}^{p}(H)=\{1\}$ is called the nilpotent p-length of $H$. If $l$ is the nilpotent $p$-length, then $\gamma_{l}^{p}(H)$ is a central subgroup of $H$ and each element has order $p$ (or $1)$.

4.19. Lemma. Assume that $H$ is a finite p-group of nilpotent $p$-length $k$ and $\alpha: H \rightarrow H$ is an automorphism such that the induced action on $H_{1}(H, \mathbb{Z} / p)$ is trivial. Then $\alpha^{p^{k-1}}=\mathrm{id}_{H}$.

Proof. This is done by induction on the nilpotent $p$-length $k$. If $k=1$, then $H \cong H_{1}(H, \mathbb{Z} / p)$, and the assertion follows by assumption.

If $k>1$, consider the group $H^{\prime}:=H / \gamma_{k}^{p}(H)$. This is a finite $p$-group of nilpotent $p$-length $k-1$. Moreover, the projection $H \rightarrow H^{\prime}$ induces an isomorphism $H_{1}(H, \mathbb{Z} / p) \rightarrow H_{1}\left(H^{\prime}, \mathbb{Z} / p\right)$. Let $\bar{\alpha}: H^{\prime} \rightarrow H^{\prime}$ be the automorphism induced by $\alpha$. By induction, $(\bar{\alpha})^{p^{k-2}}=\operatorname{id}_{H^{\prime}}$.

Set $\beta:=\alpha^{p^{k-2}}$. We show $\beta^{p}=\operatorname{id}_{H}$, which immediately implies the result. 
Since $\beta$ acts trivially on $H / \gamma_{k}^{p}(H)$, for each $x \in H$ we have $\beta(x)=x \phi(x)$ with $\phi(x) \in \gamma_{k}^{p}(H)$. Now

$$
x y \phi(x y)=\beta(x y)=\beta(x) \beta(y)=x \phi(x) y \phi(y)=x y \phi(x) \phi(y),
$$

using the fact that $\gamma_{k}^{p}(H)$ is central in $H$. Therefore $\phi: H \rightarrow \gamma_{n-1}(H)$ is a group homomorphism. Since $\gamma_{k}^{p}(H)$ is an abelian $\mathbb{Z} / p$-vector space and lies in the kernel of the projection $H \rightarrow H_{1}(H, \mathbb{Z} / p)$ (here we use $k>1$ ), the restriction of $\phi$ to $\gamma_{k}^{p}(H)$ is trivial, and therefore the restriction of $\beta$ to $\gamma_{k}^{p}(H)$ is the identity. It follows that $\beta^{r}(x)=x \cdot(\phi(x))^{r}$. In particular $\beta^{p}(x)=x$, since $\phi(x)$ like each element of $\gamma_{k}^{p}(H)$ has order $p$.

4.20. Lemma. Let $H$ be a central subgroup of the group $G$ such that $H$ is a finite $p$ group and $G / H$ is cohomologically p-complete. Then $G$ contains a normal subgroup $N$ such that $G / N$ is a finite p-group, and $H \cap N=\{1\}$.

Proof. We prove this by induction on the nilpotent $p$-length $k$ of $H$. Set $Q=G / H$ and assume first that $k=1$. Then $H=H_{1}(H, \mathbb{Z} / p) \cong H^{1}(H, \mathbb{Z} / p)$. By Lemma 4.12, the exact sequence $1 \rightarrow H \rightarrow G \rightarrow Q \rightarrow 1$ maps to the exact sequence of pro-p-completions $1 \rightarrow \bar{H} \rightarrow \hat{G}^{p} \rightarrow \hat{Q}^{p} \rightarrow 1$, where, since $H$ is a finite $p$-group, $\bar{H}$ is a quotient of $H$. The spectral sequences for the cohomology of such extensions give rise to exact sequences in low degrees, and because of naturality we get the following diagram of exact sequences:

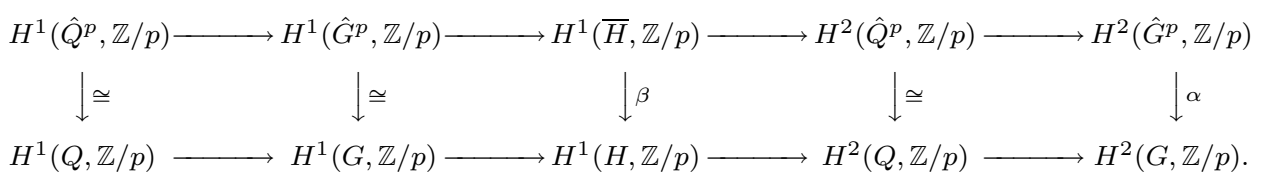

Note that in general, the middle term is $H^{1}(H, \mathbb{Z} / p)^{G}$, the $G$-fixed set of $H^{1}(H, \mathbb{Z} / p)$ (and similarly $H^{1}(\bar{H}, \mathbb{Z} / p)^{\hat{G}^{p}}$ ). In our case, by assumption, the action of $G$ on $H$ is trivial, and this implies that $G$ acts trivially on $H^{1}(H, \mathbb{Z} / p)$ and $\hat{G}^{p}$ acts trivially on $H^{1}(\bar{H}, \mathbb{Z} / p)$, so that we get the above diagram exactly.

By Lemma 4.5 the first two homomorphism are isomorphisms, and $\beta$ is injective. $Q$ being cohomologically $p$-complete explains why the fourth homomorphism is an isomorphism. The 5 -lemma now implies that $\beta$ is an isomorphism.

Since, by assumption, $H \cong H^{1}(H, \mathbb{Z} / p)$ and $\bar{H}$ is a quotient of $H$, we also have $\bar{H} \cong H^{1}(\bar{H}, \mathbb{Z} / p) \underset{\beta}{\cong} H^{1}(H, \mathbb{Z} / p) \cong H$. By Lemma 4.12 this means that for each normal subgroup $U$ of $H$ of $p$-power index, in particular for $\{1\}$, we find a normal subgroup $V$ of $G$ such that $G / V$ is a finite $p$-group and such that $V \cap H \subset U$. This proves the statement in the case $k=1$.

If the nilpotent $p$-length $k$ is bigger than 1 , consider

$$
L:=\gamma_{2}^{p}(H)=\operatorname{ker}\left(H \rightarrow H_{1}(H, \mathbb{Z} / p)\right) .
$$

Since this is a characteristic subgroup of $H$, we get an exact sequence

$$
1 \rightarrow H / L \rightarrow G / L \rightarrow Q \rightarrow 1,
$$

with $H_{1}(H / L, \mathbb{Z} / p) \cong H / L$, and $G / L$ still acting trivially on $H_{1}(H / L, \mathbb{Z} / p) \cong$ $H_{1}(H, \mathbb{Z} / p)$. As we have just proved, we then find a normal subgroup $U$ of $G / L$ which has finite $p$-power index in $G / L$ and such that $U \cap H / L=\{1\}$. Let $\pi: G \rightarrow$ $G / L$ be the canonical projection, and $V:=\pi^{-1}(U)$ the inverse image of $U$ in $G$. Then $G / V$ is a finite $p$-group, and $V \cap H \subset L=\gamma_{2}^{p}(H)$. Set $Q^{\prime}:=V H / H$. This is 
a normal subgroup of $Q$, and $Q / Q^{\prime}$ is a finite $p$-group. By Proposition 4.16, $Q^{\prime}$ is cohomologically $p$-complete. We get an exact sequence

$$
1 \rightarrow L \rightarrow V \rightarrow Q^{\prime} \rightarrow 1 \text {. }
$$

Of course, $V$ still acts trivially on $L$. Moreover, the nilpotent $p$-length of $L=\gamma_{2}^{p}(H)$ is strictly smaller than $k$, the nilpotent $p$-length of $H$. By induction, we therefore find a normal subgroup $W$ of $V$ such that $V / W$ is a finite $p$-group and such that $L \cap W=\{1\}$. By Lemma4.11, we can replace $W$ by a smaller normal subgroup $W^{\prime}$ such that $V / W^{\prime}$ is still a finite $p$-group, such that of course also still $L \cap W^{\prime}=\{1\}$, and such that $W^{\prime}$ is normal in $G$. It follows that $G / W^{\prime}$ is a finite $p$-group. Since $W^{\prime} \leq V$ and $V \cap H \leq L$, we finally get from $W^{\prime} \cap L=\{1\}$ that $W^{\prime} \cap H=\{1\}$, as desired.

4.21. Remark. Though the condition that $H$ is central in $G$ is rather special, Lemma 4.20 is still an important first step in our proof of Theorem 4.15.

4.22. Lemma. Assume that $1 \rightarrow H \rightarrow G \rightarrow Q \rightarrow 1$ is an exact sequence of groups, $H$ is a finite p-group, each element of $G$ acts nilpotently on $H_{1}(H, \mathbb{Z} / p)$, and $Q$ is cohomologically p-complete. Then $G$ contains a normal subgroup $V$ such that $G / V$ is a finite p-group, and $H \cap V=\{1\}$.

Proof. The action of $G$ on $H$ is a homomorphism from $G$ to the finite group $\operatorname{Aut}(H)$. Let $N$ be the kernel of this homomorphism. We claim that $G / N$ is a finite $p$-group. Since $G / N$ is finite, it suffices to show that the order of each element of $G / N$ is a power of $p$. Fix $g \in G$. Lemma 4.17 and Lemma 4.19 together imply that $g^{p^{k}}$ acts trivially on $H$ if $k$ is sufficiently big, i.e. $g^{p^{k}} \in N$. Since $g$ was arbitrary, the order of each element of $G / N$ is a power of $p$.

Set $Q^{\prime}:=N H / H$. This is a normal subgroup of $Q$, and $Q / Q^{\prime}$ is a finite $p$ group. By Proposition 4.16, $Q^{\prime}$ is cohomologically $p$-complete. Moreover, in the exact sequence $1 \rightarrow H \cap N \rightarrow N \rightarrow Q^{\prime} \rightarrow 1$, by construction $N$ acts trivially on $H \cap N$. By Lemma 4.20, we can find a normal subgroup $U$ of $N$ such that $N / U$ is a finite $p$-group and such that $H \cap U=(H \cap N) \cap U=\{1\}$. Moreover by Lemma 4.11, we can replace $U$ by a smaller subgroup $V$ such that $V \triangleleft G$ and $N / V$ is a finite $p$-group. Then $G / V$ is also a finite $p$-group, which finishes the proof because obviously $H \cap V=\{1\}$.

4.23. Proposition. Assume that $1 \rightarrow H \rightarrow G \rightarrow Q \rightarrow 1$ is an exact sequence of groups, $H$ is finitely generated, each element of $G$ acts unipotently on $H_{1}(H, \mathbb{Z} / p)$, and $Q$ is cohomologically $p$-complete. Then we get an exact sequence of pro-p completions

$$
1 \rightarrow \hat{H}^{p} \rightarrow \hat{G}^{p} \rightarrow \hat{Q}^{p} \rightarrow 1 .
$$

Proof. By Lemma4.12, we only have to prove that for each normal subgroup $U$ of $H$ such that $H / U$ is a finite $p$-group, we can find a normal subgroup $V$ of $G$ such that $G / V$ is a finite $p$-group and such that $V \cap H \leq U$. Fix such a $U$. Since $H$ is finitely generated, by Lemma 4.11, we can replace $U$ by a smaller characteristic subgroup $V$ such that $V \leq U$ and $H / V$ is a finite $p$-group. We obtain the exact sequence $1 \rightarrow H / V \rightarrow G / V \rightarrow Q \rightarrow 1$. Since $H_{1}(H / V, \mathbb{Z} / p)$ is a quotient of $H_{1}(H, \mathbb{Z} / p)$, each element of $G / V$ acts unipotently on $H_{1}(H, \mathbb{Z} / p)$ (without loss of generality we can even assume that $V \leq \gamma_{2}^{p}(H)$, and then $\left.H_{1}(H, \mathbb{Z} / p) \cong H_{1}(H / V, \mathbb{Z} / p)\right)$. Moreover, $H / V$ is a finite $p$-group. By Lemma 4.22 we find a normal subgroup $W$ of $G / V$ of 
finite $p$-power index such that $W \cap H / V=\{1\}$. Let $\tilde{W}$ be the inverse image of $W$ in $G$. Then $\tilde{W} \cap H \leq V \leq U$, and $G / \tilde{W}$ is a finite $p$-group.

Proof of Theorem 4.15. Because of Proposition 4.23, the assumptions imply that we have the exact sequence

$$
1 \rightarrow \hat{H}^{p} \rightarrow \hat{G}^{p} \rightarrow \hat{Q}^{p} \rightarrow 1 .
$$

As in the proof of Proposition 4.13, we have two compatible spectral sequences with $E_{2}^{l, q}$-terms

$$
H^{l}\left(\hat{Q}^{p}, H^{q}\left(\hat{H}^{p}, \mathbb{Z} / p\right)\right) \quad \text { or } \quad H^{l}\left(Q, H^{q}(H, \mathbb{Z} / p)\right)
$$

converging to $H^{l+q}\left(\hat{G}^{p}, \mathbb{Z} / p\right)$ or $H^{l+q}(G, \mathbb{Z} / p)$, respectively. The assumption on $H$ means that the natural map between Galois and ordinary cohomology induces an isomorphism between the coefficients in the $E_{2}$-terms, which, again by assumption, are finite $\hat{Q}^{p}$-modules. Because of the assumption on $H$ and Lemma 4.7, the natural map therefore induces an isomorphism on the $E_{2}$-term, and consequently the isomorphism between $H^{*}\left(\hat{G}^{p}, \mathbb{Z} / p\right)$ and $H^{*}(G, \mathbb{Z} / p)$ we had to establish.

If $H^{l}\left(\hat{Q}^{p}, \mathbb{Z} / p\right)$ is finite for each $l$, the same is true for each $H^{l}\left(\hat{Q}^{p}, A\right)$, for an arbitrary finite discrete $\hat{Q}^{p}$-module $A$. This means that every $E_{2}^{k, l}$ is finite. Because the spectral sequence is a first quadrant spectral sequence, the same is then true for the groups $H^{k+l}\left(\hat{Q}^{p}, \mathbb{Z} / p\right)$ to which the spectral sequence is converging.

4.24. Lemma. Let $P$ and $Q$ be two finite p-groups. Then the free product $P * Q$ is cohomologically p-complete. In particular,

$$
H^{k}\left(\widehat{P * Q}^{p}, \mathbb{Z} / p\right)=H^{k}\left(\hat{P}^{p}, \mathbb{Z} / p\right) \oplus H^{k}\left(\hat{Q}^{p}, \mathbb{Z} / p\right) \quad \text { for } k \geq 1 .
$$

Proof. By [18, Lemma 5.5] we have an exact sequence

$$
1 \rightarrow F \rightarrow P * Q \rightarrow P \times Q \rightarrow 1
$$

where $F$ is a (finitely generated, since $P$ and $Q$ are finite) free group. Since $P \times Q$ is a finite $p$-group and $F$ is cohomologically $p$-complete by Example 4.6, Proposition 4.13 implies that $P * Q$ is cohomologically $p$-complete, as well.

Since $H^{k}(P * Q, \mathbb{Z} / p) \cong H^{k}(P, \mathbb{Z} / p) \oplus H^{k}(Q, \mathbb{Z} / p)$ for $k \geq 1$, the corresponding statement for the pro- $p$ completions follows from cohomological $p$-completeness.

We note the following proposition for later reference in Section 5 .

4.25. Proposition. Let $G_{1}$ and $G_{2}$ be two discrete groups. Set $G:=G_{1} * G_{2}$. The inclusions $G_{k} \hookrightarrow G$ induce an isomorphism

$$
H^{k}\left(\hat{G}^{p}, \mathbb{Z} / p\right) \stackrel{\cong}{\longrightarrow} H^{k}\left(\hat{G}_{1}^{p}, \mathbb{Z} / p\right) \oplus H^{k}\left(\hat{G}_{2}^{p}, \mathbb{Z} / p\right) \quad \text { for } k \geq 1 .
$$

In particular, $G_{1}$ and $G_{2}$ are both cohomologically p-complete if and only if the same is true for $G$.

Proof. The completion $\hat{G}^{p}$ is the inverse limit of the inverse system of all quotients $G / U$ where $U$ is a normal subgroup of $G$ of finite $p$-power index. Set $U_{1}:=G_{1} \cap U$ and $U_{2}:=G_{2} \cap U$. We get a projection $\left(G_{1} / U_{1}\right) *\left(G_{2} / U_{2}\right) \rightarrow G / U$. On the other hand, $\left(G_{1} / U_{1}\right) *\left(G_{2} / U_{2}\right)$ is a quotient of $G$, so every finite $p$-group quotient of $\left(G_{1} / U_{1}\right) *\left(G_{2} / U_{2}\right)$ is a finite $p$-group quotient of $G$. It follows that we can view the inverse system which defines $\hat{G}^{p}$ as the inverse system (over $U$ ) of the inverse systems which define the pro- $p$ completion $X_{U}$ of $\left(G_{1} / U_{1}\right) *\left(G_{2} / U_{2}\right)$. The Galois 
cohomology of $\hat{G}^{p}$ by definition is the direct limit of the corresponding direct system of cohomology groups. With our reinterpretation, for $k \geq 1$,

$$
\begin{aligned}
H^{k}\left(\hat{G}^{p}, \mathbb{Z} / p\right) & =\lim _{G / U} H^{k}\left(X_{U}, \mathbb{Z} / p\right) \\
& =\lim _{G / U} H^{k}\left(G_{1} / U_{1}, \mathbb{Z} / p\right) \oplus H^{k}\left(G_{2} / U_{2}, \mathbb{Z} / p\right) .
\end{aligned}
$$

For the last equality, we use Lemma 4.24

We take the limit along the system of finite $p$-group quotients of $G$. It remains to check for each pair of normal subgroups $U_{1} \leq G_{1}$ and $U_{2} \leq G_{2}$ of $p$-power index that there is a corresponding normal subgroup $U$ of $G$ with $G_{1} \cap U=U_{1}$ and $G_{2} \cap U=U_{2}$. But for such $U_{1}$ and $U_{2}$, the kernel $U$ of the projection $G \rightarrow\left(G_{1} / U_{1}\right) \times\left(G_{2} / U_{2}\right)$ fulfills $G_{1} \cap U=U_{1}$ and $G_{2} \cap U=U_{2}$. Therefore the limit along the system of finite $p$-group quotients of $G$ is the same as the limit along the system induced from the finite $p$-group quotients of $G_{1}$ and of $G_{2}$, and consequently for $k \geq 1$

$$
\begin{aligned}
H^{k}\left(\hat{G}^{p}, \mathbb{Z} / p\right) & =\lim _{G / U} H^{k}\left(G_{1} / U_{1}, \mathbb{Z} / p\right) \oplus H^{k}\left(G_{2} / U_{2}, \mathbb{Z} / p\right) \\
& =H^{k}\left(\hat{G}_{1}^{p}, \mathbb{Z} / p\right) \oplus H^{k}\left(\hat{G}_{2}^{p}, \mathbb{Z} / p\right) .
\end{aligned}
$$

We get a commutative diagram

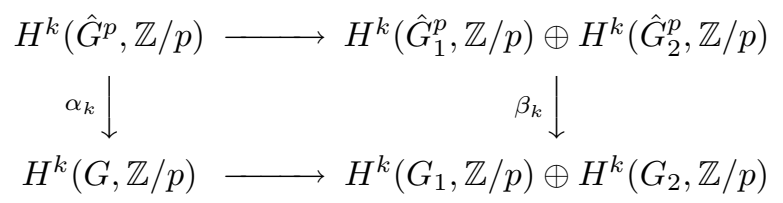

where the horizontal arrows are induced from the inclusions of the factors $G_{1}$ and $G_{2}$ in $G$, and, for $k \geq 1$, both are isomorphisms (this is a classical fact in the discrete case, and we just checked it for the pro- $p$ completions). Therefore, $\alpha_{k}$ is an isomorphism if and only if $\beta_{k}$ is an isomorphism. Since, by definition, $\alpha_{0}$ and $\beta_{0}$ always are isomorphisms, $G$ is cohomologically $p$-complete if and only if $G_{1}$ and $G_{2}$ are both cohomologically $p$-complete.

The following theorem is the technical heart of our program to find torsion-free quotients of our torsion-free finite extension, and for more general finite extensions to find quotients with as little torsion as possible.

4.27. Theorem. Assume that we have an exact sequence

$$
1 \rightarrow H \rightarrow G \rightarrow Q \rightarrow 1,
$$

where $Q$ is a finite p-group and $H$ is cohomologically $p$-complete and has a finite classifying space BH. Assume that the induced exact sequence

$$
1 \rightarrow \hat{H}^{p} \rightarrow \hat{G}^{p} \rightarrow Q \rightarrow 1
$$

splits. Then the original sequence also splits, in particular we have an embedding $Q \hookrightarrow G$.

The idea of the proof is that the splitting of the pro- $p$ completions induces a splitting of cohomology. But the cohomology for the completions and the original groups coincide (since we assume that $H$ is cohomologically $p$-complete), so that the original map splits after taking cohomology. With a spectral sequence argument, the same follows on the level of stable cohomotopy, and this induces the splitting on the group level. 
To make this precise, some preparation is necessary.

The next theorem with proof was communicated to us by Alejandro Adem. We thank him for his very prompt and kind answer to our questions.

4.28. Theorem (Adem). Let $H$ denote a discrete group of finite cohomological dimension. Suppose that we are given an extension

$$
1 \rightarrow H \rightarrow G \stackrel{f}{\rightarrow} Q \rightarrow 1
$$

where $Q$ is a finite p-group. For a space $Y$, let $\tilde{\pi}_{S}^{0}(Y)$ denote its degree zero reduced stable cohomotopy group.

The extension above splits if and only if the epimorphism $f: G \rightarrow Q$ induces an injection $\tilde{f}^{*}: \tilde{\pi}_{S}^{0}(B Q) \rightarrow \tilde{\pi}_{S}^{0}(B G)$.

Proof. Clearly, if the extension splits, then the map induced on reduced stable cohomotopy is injective. Now let us assume that this map is injective. Then the map $f^{*}$ induced in unreduced cohomotopy is also injective, since we only add the summand id: $\pi_{S}^{0}(p t) \rightarrow \pi_{S}^{0}(p t)$.

By a construction due to Serre [7, Theorem 3.1 and following exercise] we can find a finite dimensional CW-complex $X$ with an action of $G$ and such that $B G \simeq$ $X / H \times{ }_{Q} E Q$. In particular, we can identify the map $f^{*}$ with the map induced in stable cohomotopy by the fibration $X / H \times{ }_{Q} E Q \rightarrow B Q$.

The main result in [25] implies that, if $f^{*}$ is injective, then the action of $Q$ on $X / H$ has a fixed point. In particular, the fibration above has a section and hence the group extension must split.

We want to apply this result to the extension given in Theorem 4.27 As an intermediate step we use stable cohomotopy of the pro- $p$ completions and localization of these cohomotopy groups at the prime $p$.

4.3. Finiteness conditions for classifying spaces. At several points, we will have to assume finiteness conditions on the classifying spaces. We collect a few results about this here. Since we are only interested in (co)homology, we can do all of our constructions up to homotopy.

4.29. Definition. A CW-complex is called finite, if it consists of finitely many cells. It is called of finite type, if each finite dimensional subcomplex is finite.

4.30. Lemma. If $F \rightarrow E \rightarrow B$ is a fibration such that $F$ and $B$ are homotopy equivalent to $C W$-complexes of finite type, then the same is true for $E$.

Let $1 \rightarrow H \rightarrow G \rightarrow P \rightarrow 1$ be an exact sequence of groups, where $B H$ and $B P$ are $C W$-complexes of finite type. Then the same is true for $B G$.

Proof. The first statement follows from [39, 1.3 and 1.8], and also from [37, Chapter 14]. An explicit account can be found in [6, 2.2.1].

Then apply this to the fibration $B H \rightarrow B G \rightarrow B P$.

\subsection{Generalized Galois cohomology of profinite groups.}

4.31. Definition. Assume that $h^{*}$ is a generalized cohomology theory and $\mathfrak{Q}$ is a profinite group. We set

$$
h^{*}(\mathfrak{Q}):=\operatorname{dirlim}_{|\mathfrak{Q} / N|<\infty} h^{*}(\mathfrak{Q} / N)
$$

where $h^{*}(\mathfrak{Q} / N):=h^{*}(B[\mathfrak{Q} / N])$ is the cohomology of the classifying space of the finite quotient $\mathfrak{Q} / N$. 
Filtration by skeleta. Let $X$ be a CW-complex and $h^{*}$ an arbitrary generalized cohomology theory. Let $X^{0} \subset X^{1} \subset \cdots \subset X$ be the filtration of $X$ by its skeleta. Let $F_{k}^{*}(X)$ for $k \in \mathbb{N}$ be the kernel of the induced map $h^{*}(X) \rightarrow h^{*}\left(X^{(k)}\right)$. Set $F_{0}^{k}(X):=h^{k}(X)$. This way, we get a filtration

$$
h^{*}(X)=F_{0}^{*}(X) \supset F_{1}^{*}(X) \supset \cdots \supset\{0\} .
$$

If $X$ is finite dimensional, then this stabilizes after finitely many steps. More precisely, $F_{\operatorname{dim} X}^{k}(X)=\{0\}$, since $X^{\operatorname{dim} X}=X$. If $X$ is not finite dimensional the sequence does not necessarily stabilize. It is not even clear whether $\bigcap_{k \in \mathbb{N}} F_{k}^{*}(X)=$ $\{0\}$. We will later study conditions under which the last assertion is true.

Note, however, that every continuous map (being homotopic to a cellular map) induces maps which preserve these filtrations. In particular, by passing to direct limits, we get, for a profinite group $\mathfrak{Q}$, a filtration

$$
h^{*}(\mathfrak{Q})=F_{0}^{*}(\mathfrak{Q}) \supset F_{1}^{*}(\mathfrak{Q}) \supset \cdots .
$$

4.32. Lemma. Definition 4.31 defines a functor from the category of profinite groups to filtered graded abelian groups. In particular, a split projection $G \rightarrow \mathfrak{Q}$ induces a split injection $h^{*}(\mathfrak{Q}) \rightarrow h^{*}(G)$. It also induces split injections of the filtration spaces $F_{k}^{*}(\mathfrak{Q}) \rightarrow F_{k}^{*}(G)$ and of the quotients $F_{l}^{*}(\mathfrak{Q}) / F_{k}^{*}(\mathfrak{Q}) \rightarrow F_{l}^{*}(G) / F_{k}^{*}(G)$ if $l \leq k$.

If for a prime number $p$ the group $\mathfrak{Q}$ is the pro- $p$ completion of a discrete group $G$, then the inclusion induces a map $h^{*}(\mathfrak{Q}) \rightarrow h^{*}(G)$.

Proof. A map between profinite groups is the same as a map between the inverse system of finite quotients, giving maps between the direct systems of cohomology groups. This is functorial. Because of the splitting, we can also pass to the quotients $F_{l} / F_{k}$.

The last statement, about the pro- $p$ completion, follows because we get a consistent system of maps to all the finite quotients of $\mathfrak{Q}$, which induce a corresponding map from the direct system of cohomology groups to $h^{*}(G)$.

4.5. Localization. We collect a few well known results about localization in the following proposition (compare e.g. [12, Chapter 15.4] and [5, p. 67 in II.2.4]), which we are going to use later.

4.33. Proposition. Let $p$ be a prime number and $\mathbb{Z}_{(p)}:=\{a / b \in \mathbb{Q} \mid p \nmid b\}$ the localization of $\mathbb{Z}$ at $p$. For an arbitrary abelian group $M, M_{(p)}:=M \otimes_{\mathbb{Z}} \mathbb{Z}_{(p)}$ is the localization of $M$ at $p$. Then

- Localization at $p$ is an exact functor.

- The kernel of the map $M \rightarrow M_{(p)}: m \mapsto m \otimes 1$ consists of those elements in $M$ which are of finite order not divisible by $p$. In particular, if $M$ is a torsion module, this map is the projection onto the p-primary component of $M$.

- Localization commutes with tensor products.

- Localization commutes with direct limits.

4.6. Spectral sequences. A (cohomological) spectral sequence $\left(E_{*}^{*, *}\right)$ consists of abelian groups

$$
E_{r}^{s, t} \quad \text { for } r \geq 2, s, t \in \mathbb{Z}
$$

with homomorphisms, the so called differentials,

$$
d_{r}^{s, t}: E_{r}^{s, t} \rightarrow E_{r}^{s+r, t-r+1}, \quad \text { which satisfy } d_{r}^{s, t} \circ d_{r}^{s-r, t+r-1}=0,
$$


and with isomorphisms

$$
\Phi_{r}^{s, t}: \operatorname{ker}\left(d_{r}^{s, t}\right) / \operatorname{im}\left(d_{r}^{s-r, t+r-1}\right) \stackrel{\cong}{\rightrightarrows} E_{r+1}^{s, t} \quad \text { for } r \geq 2, s, t \in \mathbb{Z} .
$$

In other words, each page $E_{r}^{*, *}(r \geq 2)$ consists of chain complexes, and $E_{r+1}^{*, *}$ is obtained as the cohomology of $\left(E_{r}^{*, *}, d_{r}^{*, *}\right)$.

A map of spectral sequences $f_{*}^{*, *}: E_{*}^{*, *} \rightarrow F_{*}^{*, *}$ consists of homomorphisms $f_{r}^{s, t}: E_{r}^{s, t} \rightarrow F_{r}^{s, t}$ which are compatible with the differentials $d_{*}^{*, *}$ and the isomorphisms $\Phi_{*}^{*, *}$.

If $E_{2}^{s, t}=0$ whenever $s<0$, then for all $r \geq 2$ we have $E_{r}^{s, t}=0$ if $s<0$. Consequently, for $r>s$ the domain of $d_{r}^{s-r, t+r-1}$ is zero and hence $\operatorname{im}\left(d_{r}^{s-r, t+r-1}\right)=$ 0 and therefore

$$
E_{r+1}^{s, t}=\operatorname{ker}\left(d_{r}^{s, t}\right) \subset E_{r}^{s, t} \quad \text { for } r>s .
$$

In this case, we define $E_{\infty}^{s, t}:=\bigcap_{r>s} E_{r}^{s, t}$.

We denote a spectral sequence $\left(E_{*}^{*, *}\right)$ as a fourth quadrant spectral sequence if $E_{2}^{s, t}=0$ for $s<0$ or $t>0$. Note that for a fourth quadrant spectral sequence, $E_{\infty}^{s, t}$ is defined for all $s, t \in \mathbb{Z}$.

Let $h^{p}=F_{0}^{p} \supset F_{1}^{p} \supset F_{2}^{p} \supset \cdots$ be a collection of graded groups, $p \in \mathbb{Z}$. We say a spectral sequence $\left(E_{*}^{*, *}\right)$ for which $E_{\infty}^{s, t}$ is defined (e.g. a fourth quadrant spectral sequence) converges to $h^{*}$, if

$$
E_{\infty}^{s, t} \cong F_{s}^{s+t} / F_{s+1}^{s+t} \quad \text { for all } s, t \in \mathbb{Z} .
$$

If $F_{k}^{p}=0$ for $k$ sufficiently large, this determines (up to extension problems) $h^{p}$. This is not necessarily the case if only $\bigcap_{k \in \mathbb{N}} F_{k}^{p}=0$.

4.34. Lemma. Assume that $E_{r}^{s, t}$ is a fourth quadrant spectral sequence converging to the graded groups $h^{s+t}=H_{0}^{s+t} \supset H_{1}^{s+t} \supset \cdots$. Let $\mathcal{P}$ be a property of abelian groups which is preserved under passage to subgroups, quotient groups, and under extensions (i.e. if in an exact sequence $1 \rightarrow U \rightarrow G \rightarrow Q \rightarrow 1$ the groups $U$ and $Q$ have property $\mathcal{P}$, then $G$ also has property $\mathcal{P})$. Examples of such properties are the property of being a finite group and the property of being a p-group ( $p$ a prime number):

(1) If, for some $s, t \in \mathbb{Z}, E_{2}^{s, t}$ has property $\mathcal{P}$, then $E_{\infty}^{s, t}$ also has property $\mathcal{P}$.

(2) If $s, t \in \mathbb{Z}$ with $s+t=n$ implies that $E_{2}^{s, t}$ has property $\mathcal{P}$, then $h^{n} / H_{k}^{n}$ has property $\mathcal{P}$ for every $k \in \mathbb{N}$.

(3) If, in addition, for the given $n \in \mathbb{Z}$ there is some $k \in \mathbb{N}$ such that $H_{k}^{n}=0$, then $h^{n}$ has property $\mathcal{P}$.

(4) Assume that $F_{r}^{s, t}$ is another fourth quadrant spectral sequence converging to the graded group $g^{s+t}=G_{0}^{s+t} \supset G_{1}^{s+t} \supset \cdots$. Assume that we have a map of spectral sequences $f_{r}^{s, t}: E_{r}^{s, t} \rightarrow F_{r}^{s, t}$ such that $f_{\infty}^{s, t}: E_{\infty}^{s, t} \rightarrow F_{\infty}^{s, t}$ is injective for every $s, t \in \mathbb{Z}$. Suppose, moreover, that this map is compatible with a corresponding map of graded groups $h^{*} \rightarrow g^{*}$. Then this induces injective maps

$$
h^{n} / H_{k}^{n} \hookrightarrow g^{n} / G_{k}^{n} \quad \text { for all } k \in \mathbb{N}, n \in \mathbb{Z} .
$$

Proof. First observe that by induction on $r$ every $E_{r}^{s, t}$ —as a sub-quotient of $E_{r-1}^{s, t}-$ has property $\mathcal{P}$, and the same is then also true for $E_{\infty}^{s, t} \subset E_{s}^{s, t}$. Using the extensions

$$
1 \rightarrow H_{s}^{s+t} / H_{s+1}^{s+t} \rightarrow h^{s+t} / H_{s+1}^{s+t} \rightarrow h^{s+t} / H_{s}^{s+t} \rightarrow 1,
$$


and the isomorphism $H_{s}^{s+t} / H_{s+1}^{s+t} \cong E_{\infty}^{s, t}$, it follows by induction that $h^{s+t} / H_{k}^{s+t}$ has property $\mathcal{P}$ for every $k \in \mathbb{N}$. If $H_{k}^{s+t}=0$, then $h^{s+t}=h^{s+t} / H_{k}^{s+t}$, so that under this assumption $h^{s+t}$ also has property $\mathcal{P}$.

Given a map between short exact sequences of abelian groups

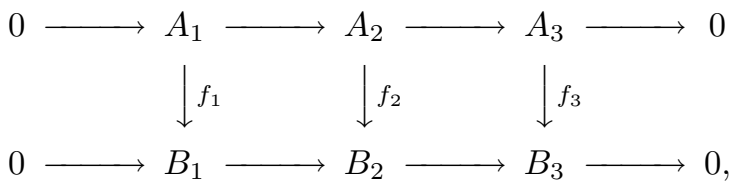

an easy diagram chase shows that $f_{2}$ is injective if both $f_{1}$ and $f_{3}$ are injective. The injectivity in (4) of $h^{n} / H_{k}^{n} \hookrightarrow g^{n} / G_{k}^{n}$ follows inductively from this principle applied to the exact sequences (4.35) and their counterparts for $g^{*}$.

4.36. Lemma. Let $E_{r}^{s, t}$ be a cohomological spectral sequence.

(1) Given $r, s, t$, there is a finite set $V(r, s, t) \subset \mathbb{Z} \times \mathbb{Z}$ such that $E_{r}^{s, t}$ is determined by $E_{2}^{x, y}$ for $(x, y) \in V(r, s, t)$. More precisely, if $f_{r}^{s, t}: E_{r}^{s, t} \rightarrow F_{r}^{s, t}$ is a map of spectral sequences such that $f_{2}^{x, y}: E_{2}^{x, y} \rightarrow F_{2}^{x, y}$ is an isomorphism for all $(x, y) \in V(s, t, r)$, then $f_{r}^{s, t}: E_{r}^{s, t} \rightarrow F_{r}^{s, t}$ is also an isomorphism.

(2) If we restrict ourselves to the category of fourth quadrant spectral sequences and if $s+t \leq 0$, then we can choose $V(r, s, t)$ as in $(1)$ such that $(x, 0) \notin$ $V(r, s, t)$ if $x \geq 1$.

Proof. We prove (1) by induction. The statement is trivial for $r=2$. Given $r>2$, $E_{r}^{s, t}$ depends only on the three groups $E_{r-1}^{s-r+1, t+r-2}, E_{r-1}^{s, t}$ and $E_{r-1}^{s+r-1, t+r-2}$. By induction, each of these depends only on finite set $V_{1}, V_{2}, V_{3} \subset \mathbb{Z}^{2}$, and we can choose $V$ to be the union of these.

To prove (2), assume from now on that all spectral sequences are fourth quadrant spectral sequences. Define $Z_{2}:=\{(x, 0) \mid x \geq 1\} \subset \mathbb{Z} \times \mathbb{Z}$ and inductively

$$
Z_{r+1}:=\left(Z_{r} \cup d_{r}\left(Z_{r}\right) \cup d_{r}^{-1}\left(Z_{r}\right)\right) \cap\left(\mathbb{Z}_{\geq 0} \times \mathbb{Z}_{\leq 0}\right) \quad \text { for } r \geq 2,
$$

with $d_{r}(x, y):=(x+r, y-r+1)$. This is the "zone of influence" of $Z_{2}$ : if $(s, t) \notin Z_{r}$, then $E_{r}^{s, t}$ does not change if only $E_{2}^{x, y}$ with $(x, y) \in Z_{2}$ is changed.

To prove (2), it suffices to show that $(s, t) \notin Z_{r}$ if $s+t \leq 0$. Assume that this last assertion is wrong and let $r$ be minimal such that there is $(s, t) \in Z_{r}$ with $s+t \leq 0$. It follows from the definition of $Z_{r}$ that there are sequences

$$
2 \leq r_{1}<r_{2}<\cdots<r_{n} \quad \text { and } \quad \epsilon_{1}, \ldots, \epsilon_{n} \in\{1,-1\},
$$

and $x \geq 1$ such that for every $k=1, \ldots, n$

$$
\left(x+\epsilon_{1} r_{1}+\cdots+\epsilon_{k} r_{k},\left(1-r_{1}\right) \epsilon_{1}+\left(1-r_{2}\right) \epsilon_{2}+\cdots+\left(1-r_{k}\right) \epsilon_{k}\right) \in Z_{r_{k}+1},
$$

and

$$
(s, t)=\left(x+\epsilon_{1} r_{1}+\cdots+\epsilon_{n} r_{n},\left(1-r_{1}\right) \epsilon_{1}+\left(1-r_{2}\right) \epsilon_{2}+\cdots+\left(1-r_{n}\right) \epsilon_{n}\right) .
$$

Let $i_{1}<i_{2}<\cdots$ be the ordered sequence of indices with $\epsilon_{i_{k}}=1, j_{1}<j_{2}<\cdots$ similarly with $\epsilon_{j_{k}}=-1$. The inequality $s+t \leq 0$ translates to

$$
\epsilon_{1}+\cdots+\epsilon_{n} \leq-x<0 .
$$

On the other hand, since we consider spectral sequences which are zero in the upper half-plane and define $Z_{r}$ accordingly,

$$
\left(1-r_{1}\right) \epsilon_{1}+\cdots+\left(1-r_{k}\right) \epsilon_{k} \leq 0 \quad \text { for every } k=1, \ldots, n .
$$


This implies that $\epsilon_{1}=1$, i.e. $i_{1}=1$, and, using monotonicity of the $r_{i}$, that $\epsilon_{2}=1$, i.e. $i_{2}=1$, and, inductively, that $i_{k+1}<j_{k}$ for $k=1,2, \ldots$ Consequently, less than half of the $\epsilon_{1}, \epsilon_{2}, \ldots, \epsilon_{n}$ are negative. But then (4.37) is violated. This gives the desired contradiction and proves $(2)$.

Proof of Theorem 4.27, From now on, we study the generalized homology theory "(reduced) stable cohomotopy" $\pi_{S}^{*}$. Consequently, if $X$ is a CW-complex or a discrete group or a profinite group, $F_{r}^{n}(X)$ will always denote the corresponding filtration group $F_{r}^{n}(X) \subset \tilde{\pi}_{S}^{n}(X)$.

In the situation of Theorem 4.27, we will consider the following commutative diagram:

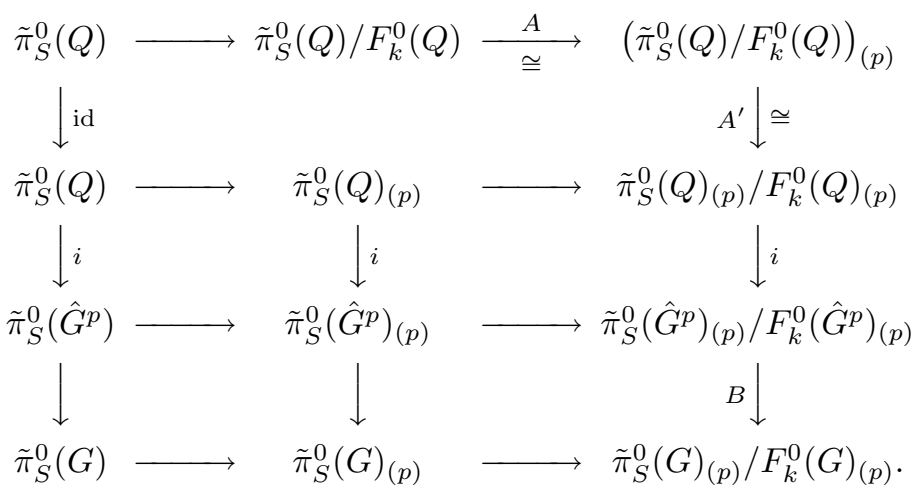

Because of Lemma 4.32 (which immediately generalized to the pro- $p$ completions) all the maps denoted with $i$ are split injective. $A^{\prime}$ is an isomorphism since localization is an exact functor.

The map $A$ is an isomorphism because of the first part of the following proposition:

4.40. Proposition. Suppose $Q$ is a finite p-group. Then the quotients

$$
\tilde{\pi}_{S}^{n}(Q) / F_{k}^{n}(Q)
$$

are finite p-groups for each $n, k \in \mathbb{Z}$. Moreover,

$$
\tilde{\pi}_{S}^{n}(Q)=\operatorname{invlim}_{k \rightarrow \infty} \tilde{\pi}_{S}^{n}(Q) / F_{k}^{n}(Q),
$$

in particular the intersection of all the $F_{k}^{n}(Q)$ is zero for each $n \in \mathbb{Z}$.

Next, we claim that the map $B$ is injective. This follows from the following Theorem 4.41 because of Proposition 4.13 . Theorem 4.41 applies since by assumption $B H$ is of finite type, so that the same is true for $B G$, using Lemma 4.30 (together with [1, Example 3.4] for the finite quotient $Q$ ).

4.41. Theorem. Suppose $G$ is cohomologically p-complete and has a classifying space of finite type. If $n \leq 0$, then the induced map

$$
B^{n}: \tilde{\pi}_{S}^{n}\left(\hat{G}^{p}\right)_{(p)} / F_{k}^{n}\left(\hat{G}^{p}\right)_{(p)} \rightarrow \tilde{\pi}_{S}^{n}(G)_{(p)} / F_{k}^{n}(G)_{(p)}
$$

is injective for each $k \geq 0$.

Given these properties of the diagram (4.39), it is easy to conclude that the induced map $\tilde{\pi}_{S}^{0}(Q) \hookrightarrow \tilde{\pi}_{S}^{0}(G)$ is injective:

If $x \in \tilde{\pi}_{S}^{0}(Q)$ is mapped to zero in $\tilde{\pi}_{S}^{0}(G)$, it is also mapped to zero in each of the quotients $\tilde{\pi}_{S}^{0}(G)_{(p)} / F_{k}^{0}(G)_{(p)}$, therefore (using injectivity of the rightmost column 
of the diagram) in each of the quotients $\tilde{\pi}_{S}^{0}(Q) / F_{k}^{0}(Q)$. Hence, $x$ is contained in $\bigcap_{k} F_{k}^{0}(Q)=\{0\}$ (where we use the second part of Proposition 4.40), i.e. $x=0$, as we had to prove. Theorem 4.27 now follows by applying Theorem 4.28,

It remains to prove Proposition 4.40 and Theorem 4.41.

First remember: if $X$ is a finite dimensional CW-complex, we have the AtiyahHirzebruch spectral sequence [28, Theorem 4.2.7] converging to $\tilde{\pi}_{S}^{s+t}(X)$, with $E_{2^{-}}$ term $E_{2}^{s, t}(X)=\tilde{H}^{s}\left(X, \pi_{-t}^{S}\right)$, where $\pi_{*}^{S}:=\pi_{*}^{S}(p t)$ denotes the coefficients of stable homotopy.

Recall the following [28, Proposition 5.1.1]:

4.42. Theorem. The coefficients of stable homotopy satisfy: $\pi_{t}^{S}$ is finite for $t>0$, $\pi_{0}^{S} \cong \mathbb{Z}$, and $\pi_{t}^{S}=0$ for $t<0$.

This implies in particular that the Atiyah-Hirzebruch spectral sequence for stable cohomotopy is a fourth quadrant spectral sequence.

We are going to apply this to the skeleta of our (infinite dimensional) classifying spaces $B G$ and $B Q$. First we study arbitrary finite or profinite groups.

4.43. Proposition. Let $Q$ be a finite group. Then we have a fourth quadrant Atiyah-Hirzebruch spectral sequence $E_{r}^{s, t}(Q)$, converging to $\tilde{\pi}_{S}^{s+t}(Q)$, with $E_{2}$-term

$$
E_{2}^{s, t}(Q)=\tilde{H}^{s}\left(Q, \pi_{-t}^{S}\right)
$$

Moreover, for fixed $s, t \in \mathbb{Z}$ and then $k$ sufficiently large, $E_{k}^{s, t}$ does not depend on $k$ (this defines $\left.E_{\infty}^{s, t}\right)$ :

$$
E_{\infty}^{s, t}=F_{s}^{s+t}(Q) / F_{s+1}^{s+t}(Q) \quad \text { and also } \quad \tilde{\pi}_{S}^{n}(Q)=\operatorname{invlim}_{k \rightarrow \infty} \tilde{\pi}_{S}^{n}(Q) / F_{k}^{n}(Q) .
$$

If $\mathfrak{Q}$ is a profinite group, then we have a fourth quadrant Atiyah-Hirzebruch spectral sequence $E_{r}^{s, t}(\mathfrak{Q})$ with $E_{2}$-term

$$
E_{2}^{s, t}(\mathfrak{Q})=\tilde{H}^{s}\left(\mathfrak{Q}, \pi_{-t}^{S}\right) .
$$

If, in addition, $H^{k}(\mathfrak{Q}, \mathbb{Z} / n)$ is finite for every $k \in \mathbb{N}_{0}, n \in \mathbb{N}$ (with trivial action of $\mathfrak{Q}$ on $\mathbb{Z} / n)$, then for each fixed $s, t \in \mathbb{Z}$ with $t \neq 0$ and for $k$ sufficiently large, $E_{k}^{s, t}$ does not depend on $k$, and we have injections of finite abelian groups

$$
F_{s}^{s+t}(\mathfrak{Q}) / F_{s+1}^{s+t}(\mathfrak{Q}) \hookrightarrow E_{\infty}^{s, t}(\mathfrak{Q}) \quad \text { for } s+t \leq 0 .
$$

Proof. If $Q$ is finite, then the existence of the spectral sequence with the given $E_{2}$-term is standard (compare the remark before [28, Theorem 4.2.7]). The issue is convergence.

Since $Q$ is finite, $\tilde{H}^{s}\left(Q, \pi_{-t}^{S}\right)$ is a torsion group [7, Corollary 10.2]. Since $\pi_{t}^{S}$ is finitely generated (Theorem 4.42) and $B Q$ is of finite type [1, Example 3.4], $\tilde{H}^{s}\left(Q, \pi_{-t}^{S}\right)$ is a finitely generated torsion group and therefore finite for every $s, t \in$ $\mathbb{Z}$. Since $E_{k}^{s, t}(Q)$ is a sub-quotient of $E_{k-1}^{s, t}(Q)$, for each $s, t \in \mathbb{Z}$ these groups eventually become constant.

We also have the corresponding Atiyah-Hirzebruch spectral sequences for the finite skeleta $B Q^{(n)}$, and these converge in the usual sense [28, Theorem 4.2.7]. Now $\tilde{H}^{s}\left(B Q^{(n)}, \pi_{-t}^{S}\right)=0$ for $s>n$ because $B Q^{(n)}$ is $n$-dimensional, and $\tilde{H}^{s}\left(Q, \pi_{-t}^{S}\right)=$ $\tilde{H}^{s}\left(B Q^{(n)}, \pi_{-t}^{S}\right)$ for $s<n$. Since $B Q^{(n)}$ is a finite CW-complex, Theorem 4.42 immediately implies that $\tilde{H}^{s}\left(B Q^{(n)}, \pi_{-t}^{S}\right)$ is finite whenever $t \neq 0$. Consequently, $E_{2}^{s, t}\left(B Q^{(n)}\right)$, and by Lemma $4.34 E_{\infty}^{s, t}\left(B Q^{(n)}\right)$ are finite except possibly for $(s, t)=$ $(n, 0)$, and are zero for $s>n$. 
By Lemma 4.34 $\tilde{\pi}_{S}^{s+t}\left(B Q^{(n)}\right)$ is finite for $s+t \neq n$, and is zero for $s+t>n$.

Observe that $B Q=\operatorname{dirlim}_{n \rightarrow \infty} B Q^{(n)}$. However, cohomotopy, as a cohomology theory, is in general not compatible with such limits. By [52, Proposition 7.66 and Remark 1 on p. 132] our finiteness results imply that here the Mittag-Leffler condition is fulfilled and therefore

$$
\tilde{\pi}_{S}^{*}(Q)=\operatorname{invlim}_{n \rightarrow \infty} \tilde{\pi}_{S}^{*}\left(B Q^{(n)}\right) .
$$

Observe that we can replace $\tilde{\pi}_{S}^{*}\left(B Q^{(n)}\right)$ by the image of $\tilde{\pi}_{S}^{*}(Q)$ in $\tilde{\pi}_{S}^{*}\left(B Q^{(n)}\right)$ without changing the inverse limit (an obvious general fact about inverse limits), and this image of course is isomorphic to $\tilde{\pi}_{S}^{*}(Q) / F_{n}^{*}(Q)$ (since $F_{n}^{*}(Q)$ is the kernel of the corresponding projection map). Therefore

$$
\tilde{\pi}_{S}^{*}(Q)=\operatorname{invlim}_{n \rightarrow \infty} \tilde{\pi}_{S}^{*}(Q) / F_{n}^{*}(Q) .
$$

Next observe that by definition we have (for $n<N$ ) the exact sequences

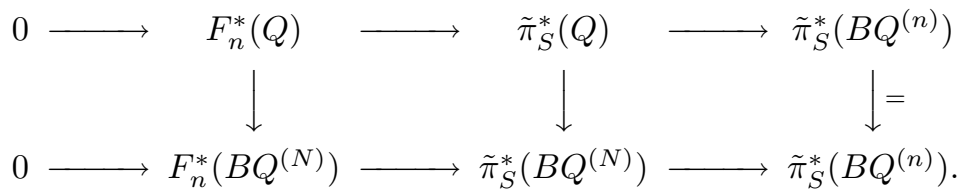

By commutativity, the images of $\tilde{\pi}_{S}^{*}(Q)$ and of $\tilde{\pi}_{S}^{*}\left(B Q^{(N)}\right)$ in $\tilde{\pi}_{S}^{*}\left(B Q^{(n)}\right)$ coincide. Now take the inverse limit of the second line for $N \rightarrow \infty$. The right-most term is constant and the middle term converges to the middle term in the first line. Since inverse limit is left exact [52, Proposition 7.63],

$$
F_{n}^{*}(Q)=\operatorname{invlim}_{N \rightarrow \infty} F_{n}^{*}\left(B Q^{(N)}\right) .
$$

We now want to establish that for $k<l$

$$
F_{k}^{*}(Q) / F_{l}^{*}(Q)=\operatorname{invlim}_{n \rightarrow \infty}\left(F_{k}^{*}\left(B Q^{(n)}\right) / F_{l}^{*}\left(B Q^{(n)}\right)\right) .
$$

By the limit result we just obtained and by [52, Proposition 7.63], we have the exact sequence

$$
0 \rightarrow F_{k}^{*}(Q) \rightarrow F_{l}^{*}(Q) \rightarrow \operatorname{invlim}_{n \rightarrow \infty} F_{k}^{*}\left(B Q^{(n)}\right) / F_{l}^{*}\left(B Q^{(n)}\right) \rightarrow \lim _{n \rightarrow \infty}{ }^{1}\left(F_{k}^{*}\left(B Q^{(n)}\right)\right) .
$$

For $s<n, F_{k}^{s}\left(B Q^{(n)}\right)$ is a sub-quotient of the cohomotopy group $\pi_{S}^{s}\left(B Q^{(n)}\right)$. We proved above that the latter one is finite. Therefore $F_{k}^{s}\left(B Q^{(n)}\right)$ is finite for $n>s$. Now [52, Remark 1 on p. 132] implies that the $\lim ^{1}$-term is zero, hence (4.44) follows.

Last observe that for $n>s$

$$
E_{2}^{s, t}(Q)=\tilde{H}^{s}\left(Q, \pi_{-t}^{S}\right)=\tilde{H}^{s}\left(B Q^{(n)}, \pi_{-t}^{S}\right)=E_{2}^{s, t}\left(B Q^{(n)}\right),
$$

since the inclusion $B Q^{(n)} \rightarrow B Q$ is an $n$-connected map.

Now fix $s, t \in \mathbb{Z}$. Since $E_{2}^{s, t}(Q)$ is finite, there is $r_{0}>s$ (depending on $s, t$ ) such that $E_{r}^{s, t}(Q)=E_{\infty}^{s, t}(Q)$ for every $r \geq r_{0}$. By Lemma 4.36 and Equation (4.45) there exists $n_{0} \geq 0$ such that

$$
E_{r_{0}}^{s, t}(Q)=E_{r_{0}}^{s, t}\left(B Q^{(n)}\right) \quad \text { for all } \quad n \geq n_{0} .
$$


Note that $n_{0}$ depends on $r_{0}, s, t$, and a priori, $n_{0}$ might become bigger if we choose a larger $r_{0}$. But, since $r_{0}>s$, we have for each $n \geq n_{0}$ a commutative diagram

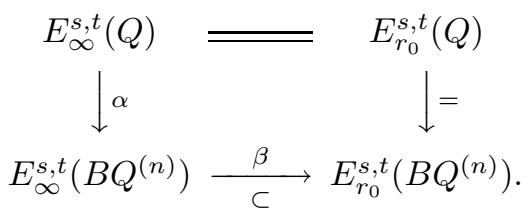

Note that $\beta$ is injective since $r_{0}>s$. Since $\beta \circ \alpha$ is an isomorphism, $\beta$ is an isomorphism, and the same is then true for $\alpha$, i.e. all groups $E_{\infty}^{s, t}(Q), E_{r}^{s, t}(Q)$, $E_{r_{0}}^{s, t}(Q), E_{r}^{s, t}\left(B Q^{(n)}\right), E_{\infty}^{s, t}\left(B Q^{(n)}\right)$ are identified, as long as $r \geq r_{0}$ and $n \geq n_{0}$.

In particular, we can write

$$
E_{r}^{s, t}(Q)=\operatorname{invlim}_{n \rightarrow \infty} E_{r}^{s, t}\left(B Q^{(n)}\right), \quad 2 \leq r \leq \infty ; s, t \in \mathbb{Z},
$$

since for fixed $s, t, r$ (including $r=\infty$ ) the sequence of groups on the right stabilizes. Since $B Q^{(n)}$ is a finite CW-complex, $F_{s}^{s+t}\left(B Q^{(n)}\right) / F_{s+1}^{s+t}\left(B Q^{(n)}\right) \cong E_{\infty}^{s, t}\left(B Q^{(n)}\right)$. By (4.44) and (4.46), both $E_{\infty}^{s, t}(Q)$ and $F_{s}^{s+t}(Q) / F_{s+1}^{s+t}(Q)$ coincide with the same group invlim $\lim _{n \rightarrow \infty} E_{\infty}^{s, t}\left(B Q^{(n)}\right)$, proving that they are equal as asserted.

If $\mathfrak{Q}$ is profinite, we define the $E_{r}$-term of the Atiyah-Hirzebruch spectral sequence for $2 \leq r<\infty$ to be the direct limit of the spectral sequences for its finite quotients $\left\{Q_{i}\right\}$. This makes sense since the direct limit functor is exact, and because of the naturality of the Atiyah-Hirzebruch spectral sequence. Since this spectral sequence is concentrated in the fourth quadrant, by Lemma $4.36 E_{r}^{s, t}(\mathfrak{Q}) \subset E_{s}^{s, t}(\mathfrak{Q})$ for $r>s$. In particular, $E_{\infty}^{s, t}(\mathfrak{Q})=\lim _{r \rightarrow \infty} E_{r}^{s, t}(\mathfrak{Q})$ makes sense, and, moreover, $E_{\infty}^{s, t}(\mathfrak{Q})=\bigcap_{r>s} E_{r}^{s, t}(\mathfrak{Q})$.

Let $Q_{i}$ be the system of finite quotients of $\mathfrak{Q}$. Note that inverse limits and direct limits do not necessarily commute. This means here that the direct limit of $E_{\infty}^{s, t}\left(Q_{i}\right)=\bigcap_{r>s} E_{r}^{s, t}\left(Q_{i}\right)$ is not necessarily $E_{\infty}^{s, t}(\mathfrak{Q})$. Exactness of direct limits implies however, using the results about finite groups proved above, that

$$
\underset{i}{\operatorname{dirlim}} E_{\infty}^{s, t}\left(Q_{i}\right)=\left(\operatorname{dirlim}_{i} F_{s}^{s+t}\left(Q_{i}\right)\right) /\left(\operatorname{dirlim}_{i} F_{s+1}^{s+t}\left(Q_{i}\right)\right)=F_{s}^{s+t}(\mathfrak{Q}) / F_{s+1}^{s+t}(\mathfrak{Q}) .
$$

Assume now that $H^{k}(\mathfrak{Q}, \mathbb{Z} / n)$ is a finite group for each $k \in \mathbb{N}_{0}, n \in \mathbb{N}$. Since these finite groups are the direct limits of the cohomology groups of the $Q_{i}$, this implies that, for fixed $n, H^{k}\left(Q_{i}, \mathbb{Z} / n\right) \rightarrow H^{k}(\mathfrak{Q}, \mathbb{Z} / n)$ is an isomorphism for all sufficiently large $i$.

Now fix $s, t \in \mathbb{Z}$ with $t \neq 0$. Observe that $E_{r}^{s, t}(\mathfrak{Q})$ is a sub-quotient of $H^{s}\left(\mathfrak{Q}, \pi_{-t}^{S}\right)$ and therefore, since $t \neq 0$, is finite by Theorem 4.42 and our finiteness assumption. Consequently, there is some $R=R(s, t)>s$ such that $E_{r}^{s, t}(\mathfrak{Q})=E_{R}^{s, t}(\mathfrak{Q})$ for $r \geq R$. We now require additionally that $s+t \leq 0$. Following Lemma 4.36, choose a finite set $V \subset \mathbb{Z}^{2}$ such that $\left\{E_{2}^{x, y}(\mathfrak{Q}) \mid(x, y) \in V\right\}$ determines $E_{R}^{s, t}(\mathfrak{Q})$. Since $s+t \leq 0$ and all our spectral sequences are concentrated in the fourth quadrant, we can assume that $(x, 0) \notin V$ if $x>0$. By the above remark, if $i$ is sufficiently large (depending on $s, t, R$ )

$$
E_{2}^{x, y}\left(Q_{i}\right)=\tilde{H}^{x}\left(Q_{i}, \pi_{-y}^{S}\right) \rightarrow \tilde{H}^{x}\left(\mathfrak{Q}, \pi_{-y}^{S}\right)=E_{2}^{x, y}(\mathfrak{Q})
$$

is an isomorphism for all $(x, y) \in V$. Since $\pi_{0}^{S} \cong \mathbb{Z}$ and we did only make an assumption about cohomology with finite coefficients, we carefully have to avoid $y=0$, except that $\tilde{H}^{0}=0$, so $x=0=y$ is permitted. The choice of $V$ implies 
that for these sufficiently large $i, E_{R}^{s, t}\left(Q_{i}\right) \rightarrow E_{R}^{s, t}(\mathfrak{Q})$ is also an isomorphism. We picked $R>s$, hence $E_{\infty}^{s, t}\left(Q_{i}\right) \subset E_{R}^{s, t}\left(Q_{i}\right)=E_{\infty}^{s, t}(\mathfrak{Q})$. Passage to the limit gives

$$
\operatorname{dirlim}_{i} E_{\infty}^{s, t}\left(Q_{i}\right)=F_{s}^{s+t}(\mathfrak{Q}) / F_{s+1}^{s+t}(\mathfrak{Q}) \subset E_{\infty}^{s, t}(\mathfrak{Q}) .
$$

Observe that we have now essentially proved Proposition 4.40, It only remains to remark that if $Q$ is a finite $p$-group, then $\tilde{H}^{*}(Q, A)$ is a finite (abelian) $p$-group for arbitrary finitely generated coefficients (compare [1, Corollary 5.4]). By Lemma 4.34, $\tilde{\pi}_{S}^{s}(Q) / F_{k}^{s}(Q)$ is a finite (abelian) $p$-group for each $k \in \mathbb{N}_{0}$ and $s \in \mathbb{Z}$.

We now study an infinite group $G$.

4.47. Proposition. Assume that $G$ is a discrete group with classifying space $B G$ of finite type. Then we have a fourth quadrant Atiyah-Hirzebruch spectral sequence $E_{r}^{s, t}(G)$ for stable cohomotopy with $E_{2}^{s, t}(G)=\tilde{H}^{s}\left(G, \pi_{-t}^{S}\right)$.

The following partial convergence results are true: for each fixed $s, t \in \mathbb{Z}$ with $t \neq 0$ or for $s=0=t, E_{k}^{s, t}$ eventually becomes constant (this defines $E_{\infty}^{s, t}$ ); and if $s+t \leq 0$, then

$$
E_{\infty}^{s, t}(G)=F_{s}^{s+t}(G) / F_{s+1}^{s+t}(G) \quad \text { and } \quad \tilde{\pi}_{S}^{s+t}(G)=\operatorname{invlim}_{k \rightarrow \infty} \tilde{\pi}_{S}^{s+t}(G) / F_{k}^{s+t}(G) .
$$

Proof. We can apply all arguments of the proof of Proposition 4.43. When using finiteness conditions of cohomology, we have to be careful to avoid (for $s>0$ ) $\tilde{H}^{s}\left(G, \pi_{0}^{S}\right)=\tilde{H}^{s}(G, \mathbb{Z})$, and $\tilde{H}^{s}\left(G^{(n)}, \pi_{0}^{S}\right)=\tilde{H}^{s}\left(G^{(n)}, \mathbb{Z}\right)$ which were finite (if $s<$ $n$ ) with $G$ replaced by the finite group $Q$, but are not necessarily finite here. But one checks immediately that the arguments go through for the range of $s$ and $t$ stated in the proposition.

4.48. Corollary. Fix a prime number $p$. Assume that $G$ is a discrete group with classifying space $B G$ of finite type. Then we have a fourth quadrant localized AtiyahHirzebruch spectral sequence $E_{r}^{s, t}(G)_{p}$ with $E_{2}^{s, t}(G)_{p}=\tilde{H}^{s}\left(G, \pi_{-t}^{S}\right)_{(p)}$. For $t \neq 0$ this computes as

$$
E_{2}^{s, t}(G)_{p}=\tilde{H}^{s}\left(G,\left(\pi_{-t}^{S}\right)_{(p)}\right) .
$$

The following partial convergence results are true: for each fixed $s, t \in \mathbb{Z}$ with $t \neq 0$ or for $s=0=t$, if $k$ is sufficiently large, then $E_{k}^{s, t}(G)_{p}$ becomes independent of $k$ (this defines $E_{\infty}^{s, t}(G)_{p}$ ). If $s+t \leq 0$, then

$$
E_{\infty}^{s, t}(G)_{p}=F_{s}^{s+t}(G)_{(p)} / F_{s+1}^{s+t}(G)_{(p)} .
$$

If $G$ is finite, the last two statements are true for arbitrary $s, t \in \mathbb{Z}$.

If $\mathfrak{Q}$ is a profinite group, then we have a fourth quadrant localized AtiyahHirzebruch spectral sequence $E_{r}^{s, t}(\mathfrak{Q})_{p}$ with $E_{2}^{s, t}(\mathfrak{Q})_{p}=\tilde{H}^{s}\left(\mathfrak{Q}, \pi_{-t}^{S}\right)_{(p)}$.

Assume in addition that $H^{k}(\mathfrak{Q}, \mathbb{Z} / p)$ is finite for every $p$ and $k$. For $t \neq 0$, we then have

$$
E_{2}^{s, t}(\mathfrak{Q})_{p}=\tilde{H}^{s}\left(\mathfrak{Q},\left(\pi_{-t}^{S}\right)_{(p)}\right),
$$

and the following convergence results are true: for each fixed $s, t \in \mathbb{Z}$ with $t \neq 0$ or for $s=0=t$ and for $k$ sufficiently large, $E_{k}^{s, t}(\mathfrak{Q})_{p}$ becomes independent of $k$ (this defines $\left.E_{\infty}^{s, t}(\mathfrak{Q})_{p}\right)$; and if $s+t \leq 0$, then we get an injection

$$
F_{s}^{s+t}(\mathfrak{Q})_{(p)} / F_{s+1}^{s+t}(\mathfrak{Q})_{(p)} \hookrightarrow E_{\infty}^{s, t}(\mathfrak{Q})_{p} .
$$


Proof. All the localized spectral sequences are constructed by localizing the spectral sequences obtained in Proposition 4.47 or Proposition 4.43 at $p$. Observe that this construction produces spectral sequences since localization is an exact functor.

For the computation of $E_{2}$-terms (4.49) and (4.50), note that $\pi_{-t}^{S}$ is the (finite) direct sum of the $q$-primary components for all primes $q$, if $t<0$. Accordingly, $\tilde{H}^{s}\left(G, \pi_{-t}^{S}\right)$ splits as a direct sum, and the $q$-primary part of $\pi_{-t}^{S}$ gives rise to the $q$ primary part of the cohomology group. Since for abelian torsion groups localization at $p$ is just projection to the $p$-primary part, $\tilde{H}^{s}\left(G, \pi_{-t}^{S}\right)_{(p)}=\tilde{H}^{s}\left(G,\left(\pi_{-t}^{S}\right)_{(p)}\right)$. The same argument applies in the profinite case.

If $\mathfrak{Q}$ is a profinite group with $H^{k}(\mathfrak{Q}, \mathbb{Z} / p)$ finite for every $k \in \mathbb{N}_{0}$, then because of Lemma 4.7 and the statement we have just proved, $\tilde{H}^{s}\left(\mathfrak{Q}, \pi_{-t}^{S}\right)_{(p)}=\tilde{H}^{s}\left(\mathfrak{Q},\left(\pi_{-t}^{S}\right)_{(p)}\right)$ is finite for $t \neq 0$.

The remaining assertions now follow easily from Proposition 4.47 or Proposition 4.43, again using the fact that localization is an exact functor.

A priori, one has to be careful with any statement about the $E_{\infty}$-terms, because $E_{\infty}^{s, t}(G)$ is defined as the inverse limit of the $E_{r}^{s, t}(G)$, and localization does not in general commute with inverse limits. However, here every sequence defining the inverse limits stabilizes (before localization), i.e. $E_{\infty}^{s, t}(G)=E_{r}^{s, t}(G)$ for $r$ sufficiently large. The same is then true after localization. This implies $E_{\infty}^{s, t}(G)_{p}=$ $\left(E_{\infty}^{s, t}(G)\right)_{(p)}$, and, consequently, the statements for $\left(E_{\infty}\right)_{p}$ also follow from Propositions 4.47 and 4.43 and exactness of the localization functor.

The arguments given so far apply identically to a profinite group $\mathfrak{Q}$.

4.51. Remark. Note that localization does not in general commute with inverse limits. Hence we can't conclude that $\pi_{S}^{s}(G)_{(p)}=\operatorname{invlim}_{k \rightarrow \infty} \pi_{S}^{s}(G)_{(p)} / F_{k}^{s}(G)_{(p)}$ for any $s \in \mathbb{Z}$. However, we will see that the spectral sequence determines enough of the groups $\pi_{S}^{s}(G)_{(p)} / F_{k}^{s}(G)_{(p)}$.

Observe that all the spectral sequences we have constructed are natural with respect to group homomorphisms. In particular, the map to the pro- $p$ completion $c: G \rightarrow \hat{G}^{p}$ induces a map of localized spectral sequences

$$
E_{r}^{s, t}(c): E_{r}^{s, t}\left(\hat{G}^{p}\right)_{p} \rightarrow E_{r}^{s, t}(G)_{p} .
$$

If $B G$ is of finite type and $G$ is cohomologically $p$-complete, Corollary 4.48 implies that the induced map on the $E_{2}$-pages is for $t<0$

$$
\tilde{H}^{s}\left(\hat{G}^{p},\left(\pi_{-t}^{S}\right)_{(p)}\right) \rightarrow \tilde{H}^{s}\left(G,\left(\pi_{-t}^{S}\right)_{(p)}\right) .
$$

Because of cohomological $p$-completeness and Lemma 4.7 this map is an isomorphism for $t<0$ or (trivially) for $s=0=t$. If $t=0$ the coefficients are not finite so that this cannot be extended to other values of $s$ and $t$. Using Lemma 4.36, $c$ therefore induces isomorphisms on $E_{r}^{s, t}$ in particular for $s+t \leq 0$. By the convergence result of Corollary 4.48, the map hence induces injections

$$
\begin{aligned}
& F_{k-1}^{s}\left(\hat{G}^{p}\right)_{(p)} / F_{k}^{s}\left(\hat{G}^{p}\right)_{(p)} \hookrightarrow E_{\infty}^{k-1, s-k+1}\left(\hat{G}^{p}\right)_{p} \\
& \quad \cong E_{\infty}^{k-1, s-k+1}(G)_{p}=F_{k-1}^{s}(G)_{(p)} / F_{k}^{s}(G)_{(p)} .
\end{aligned}
$$

As in the proof of Lemma 4.34, we get injections

$$
\pi_{S}^{s}(c)_{k}: \pi_{S}^{s}\left(\hat{G}^{p}\right)_{(p)} / F_{k}^{s}\left(\hat{G}^{p}\right)_{(p)} \hookrightarrow \pi_{S}^{s}(G)_{(p)} / F_{k}^{s}(G)_{(p)} \quad \text { for all } k \geq 0 .
$$

This proves Theorem 4.41 and hence concludes the proof of Theorem 4.27 
4.7. Splitting of short exact sequences. We now give a condition on an extension $1 \rightarrow H \rightarrow G \rightarrow Q \rightarrow 1$ as in Theorem 4.27 which implies the splitting of the induced projection $\hat{G}^{p} \rightarrow Q$.

4.52. Lemma. Let $Q$ be a finite p-group in the exact sequence

$$
1 \rightarrow H \rightarrow G \stackrel{\pi}{\rightarrow} Q \rightarrow 1 .
$$

Assume that, among all normal subgroups of $G$ of p-power index, there is a cofinal system $U_{i} \triangleleft G$ with $U_{i} \subset H$, such that for each $i$ the homomorphism $\pi_{i}$ in

$$
G \stackrel{p_{i}}{\rightarrow} G / U_{i} \stackrel{\pi_{i}}{\rightarrow} Q
$$

has a split $s_{i}: Q \hookrightarrow G / U_{i}$. Then the pro-p completion $\hat{\pi}^{p}: \hat{G}^{p} \rightarrow Q$ has a split $s: Q \hookrightarrow \hat{G}^{p}$, too.

Proof. For each of the finitely many $q \in Q$ choose $x(q, i) \in G$ with

$$
p_{i}(x(q, i))=s_{i}(q) \in G / U_{i} .
$$

Choose a cofinal subsequence such that for every $q \in Q$ the images of $x(q, i)$ in the compact group $\hat{G}^{p}$ converge to an element $x(q) \in \hat{G}^{p}$ (limit over $i$ ). We claim that the map

$$
s: Q \rightarrow \hat{G}^{p}: q \mapsto x(q)
$$

is a split of $\hat{\pi}^{p}$. Since $\pi_{i} \circ p_{i}(x(q, i))=q \in Q$, by continuity also $\hat{\pi}^{p}(x(q))=q$. It remains to check that $s$ is a group homomorphism. Observe that for $q_{1}, q_{2} \in Q$

$$
s\left(q_{1} q_{2}^{-1}\right) s\left(q_{2}\right) s\left(q_{1}\right)^{-1}=\lim _{i} x\left(q_{1} q_{2}^{-1}, i\right) x\left(q_{2}, i\right) x\left(q_{1}, i\right)^{-1} .
$$

The topology of $\hat{G}^{p}$ and the fact that

$$
p_{i}\left(x\left(q_{1} q_{2}^{-1}, i\right) x\left(q_{2}, i\right) x\left(q_{1}, i\right)^{-1}\right)=s_{i}\left(q_{1} q_{2}^{-1}\right) s_{i}\left(q_{2}\right) s_{i}\left(q_{1}\right)^{-1}=1 \in G / U_{i}
$$

implies that this limit is 1 , i.e. $s$ indeed is a homomorphism.

4.8. Enough torsion-free quotients. In this subsection we establish conditions which guarantee that we can factorize a projection onto a finite $p$-group through a torsion-free elementary amenable quotient. This is another key ingredient in our strategy to prove the Atiyah conjecture for extensions.

4.53. Definition. Let $G$ be a discrete group. We define $\mathcal{A}_{G}$ to be the set of all normal subgroups $U \leq G$ such that $G / U$ is torsion-free and elementary amenable. $G$ is said to have enough torsion-free quotients if each normal subgroup $W \triangleleft G$, with $G / W$ a finite $p$-group for some prime number $p$, contains a subgroup $U_{W} \in \mathcal{A}_{G}$, i.e. we have a factorization

$$
G \rightarrow G / U_{W} \rightarrow G / W
$$

with $G / U_{W}$ torsion-free elementary amenable.

A subset $\mathcal{A}_{G}^{\prime}$ of $\mathcal{A}_{G}$ is called exhaustive, if $U, V \in \mathcal{A}_{G}^{\prime}$ implies $U \cap V \in \mathcal{A}_{G}^{\prime}$, if for every $n \in \mathbb{N}$ there is $U_{n} \in \mathcal{A}_{G}^{\prime}$ such that $U_{n} \leq \gamma_{n}(G)$, and if, in addition, each $W$ as above contains all but finitely many of the $U \in \mathcal{A}_{G}^{\prime}$. In particular, all the $U_{W}$ can be chosen to belong to $\mathcal{A}_{G}^{\prime}$.

We say that $G$ has enough nilpotent torsion-free quotients if we can choose an exhaustive set $\mathcal{A}_{G}^{\prime}$ such that, if $U \in \mathcal{A}_{G}^{\prime}$, then $U$ is a characteristic subgroup of $G$ and $G / U$ is nilpotent. Analogously, we define enough solvable torsion-free quotients and enough solvable-by-finite torsion-free quotients. The corresponding $\mathcal{A}_{G}^{\prime}$ then 
is called nilpotent exhaustive, solvable exhaustive, or solvable-by-finite exhaustive, respectively.

4.54. Lemma. Suppose $G$ is a finitely generated group. Let $\mathcal{X}$ be a set of normal subgroups of $G$ with torsion-free elementary amenable quotients, and such that whenever $N \triangleleft G$ and $G / N$ is a finite $p$-group we can find $U \in \mathcal{X}$ with $U \leq N$. Assume that $U, V \in \mathcal{X}$ implies $U \cap V \in \mathcal{X}$.

Then there is a nested sequence $U_{1} \geq U_{2} \geq U_{3} \geq \cdots$ with $U_{k} \leq \gamma_{k}(G)$ for every $k \in \mathbb{N}$ and such that for every finite p-group quotient $G / N$ there is $k_{N}$ with $U_{k_{N}} \leq N$. Every $U_{k}$ is an intersection $U_{k}=V_{k} \cap N_{k}$ with $V_{k} \in \mathcal{X}$ and $G / N_{k}$ is torsion-free nilpotent.

In particular, if $G$ has enough torsion-free quotients, an exhaustive subset as in Definition 4.53 exists.

Proof. Define for a prime number $p$ the subgroup $G_{p, n}$ to be the intersection of the kernels of all projections to $p$-groups which have order dividing $p^{n}$. Since $G$ is finitely generated, there are only finitely many of these. By Lemma 4.11, $G / G_{p, n}$ is a finite $p$-group.

By assumption, for each $G_{p, n}$ we find $U_{p, n} \in \mathcal{X}$ which is contained in $G_{p, n}$. For $n \in \mathbb{N}$ define $V_{n}^{\prime}$ to be the intersection of all $U_{p, k}$ with $p \leq n$ and $k \leq n$.

For $n \in \mathbb{N}$ we now want to replace the subgroup $V_{n}^{\prime}$ we just constructed by $U_{n}$ such that $U_{n} \leq \gamma_{n}(G)$. Since $G / \gamma_{n}(G)$ is nilpotent, the set $K_{n}$ of elements of finite order in $G / \gamma_{n}(G)$ is a characteristic subgroup and the quotient $\left(G / \gamma_{n}(G)\right) / K_{n}$ is torsion-free [21, Theorem 2.22]. Let $H_{n}$ be the kernel of the projection $G \rightarrow$ $\left(G / \gamma_{n}(G)\right) / K_{n}$. By [18, Theorem 2.1] for each element $1 \neq g \in K_{n}$ we find a finite p-group $Q_{g}$ ( $p$ depending on $g$ ) and a projection $G / \gamma_{n}(G) \rightarrow Q_{g}$ such that $g$ is not mapped to 1 . For each such $g$ choose $n_{g} \in \mathbb{N}$ such that we get a factorization $G \rightarrow V_{n_{g}}^{\prime} \rightarrow Q_{g}$. Set

$$
V_{n}:=V_{n}^{\prime} \cap\left(\bigcap_{k \leq n} \bigcap_{g \in K_{k}} V_{n_{g}}^{\prime}\right) \text { and } U_{n}:=V_{n} \cap H_{n} .
$$

This defines a new nested sequence of subgroups. We have seen that $G / H_{n}$ is torsion-free elementary amenable. By Lemma 4.11, the same is true for $G / U_{n}$. Fix $u \in U_{n}$. Since $U_{n} \subset H_{n}$, the image of $u$ in $G / \gamma_{n}(G)$ is contained in $K_{n}$. For $1 \neq g \in K_{n}, U_{n}$ is mapped to 1 in each of the quotients $Q_{g}$. But $g$ is not mapped to 1 , hence $u \in \gamma_{n}(G)$, i.e. $U_{n} \subset \gamma_{n}(G)$.

It is now clear that $\mathcal{A}_{G}^{\prime}:=\left\{U_{n} \mid n \in \mathbb{N}\right\}$ is an exhaustive set.

4.55. Lemma. Assume that $G$ is a finitely generated group.

If every projection $G \rightarrow Q$ onto a finite p-group $Q$ factors through a torsion-free nilpotent quotient $G \rightarrow U \rightarrow Q$, then $G$ has enough nilpotent torsion-free quotients. In particular, this depends only on the (directed) system of nilpotent quotients of $G$, ordered by inclusion of the kernels.

The corresponding statement holds with "nilpotent" replaced by "solvable" or "solvable-by-finite".

Proof. Remember first that every finite $p$-group is nilpotent. This means that the first condition in fact only depends on the system of nilpotent quotients.

Let $\mathcal{X}_{t f}$ be the system of normal subgroups of $G$ with nilpotent torsion-free quotients. We can apply Lemma 4.54 to obtain a nested sequence $U_{1} \geq U_{2} \geq \cdots$ 
with $U_{k} \leq \gamma_{k}(G)$ for every $k \in \mathbb{N}$, and such that every projection to a finite $p$-group factors through $G / U_{k}$ for suitable $k \in \mathbb{N}$. By Lemma 4.11, $U_{k} \in \mathcal{X}_{t f}$ for every $k \in \mathbb{N}$.

Now replace $U_{k}$ by $V_{k}:=\bigcap_{\alpha \in \operatorname{Aut}(G)} \alpha\left(U_{k}\right)$. Because of Lemma 4.11, $V_{k} \in \mathcal{X}_{t f}$ for every $k \in \mathbb{N}$, and these subgroups are characteristic.

The proof for enough solvable or solvable-by-finite torsion-free quotients is exactly the same, replacing "nilpotent" everywhere with "solvable" or "solvable-byfinite", respectively.

4.56. Example. If $G$ is a discrete group such that there are infinitely many lower central series quotients $G / \gamma_{n}(G)$ which are torsion-free, or infinitely many derived series quotients $G / G^{(n)}$ which are torsion-free, then we can choose the exhaustive subset $\mathcal{A}_{G}^{\prime}$ to consist of the corresponding $\gamma_{n}(G)$ or $G^{(n)}$, respectively. In particular, $G$ has enough nilpotent torsion-free quotients, or enough torsion-free solvable quotients, respectively.

4.57. Lemma. Assume that $G$ is countable, has enough solvable torsion-free quotients and is residually torsion-free solvable. Then there is a nested sequence $G \geq$ $U_{1} \geq U_{2} \geq \cdots$ of normal subgroups of $G$ such that

(1) $\bigcap_{n=1}^{\infty} U_{n}=\{1\}$,

(2) $U_{n} \leq \gamma_{n}(G)$ for every $n \in \mathbb{N}$,

(3) $G / U_{n}$ is torsion-free solvable for every $n \in \mathbb{N}$.

Proof. Since $G$ is countable and residually torsion-free solvable, there is a nested sequence $G \supset H_{1} \supset H_{2} \supset \cdots$ of normal subgroups such that $G / H_{n}$ is torsion-free solvable for every $n \in \mathbb{N}$ and such that $\bigcap_{n \in \mathbb{Z}} H_{n}=\{1\}$.

On the other hand, since $G$ has enough solvable torsion-free quotients, by definition we find $V_{n} \subset \gamma_{n}(G)$ such that $G / V_{n}$ is torsion-free solvable for each $n \in \mathbb{N}$. Then $U_{n}:=H_{n} \cap V_{n}$ will satisfy the assertions.

4.58. Definition. Let $G$ be a group and let $U$ be a subgroup of $G$. Then we set $U^{G}:=\bigcap_{g \in G} U^{g}$, the largest normal subgroup of $G$ which is contained in $U$. Note that $U^{G} \cap V^{G}=(U \cap V)^{G}$ for two subgroups $U, V$ of $G$.

Let $H$ be a subgroup of $G$ containing $U$ as a normal subgroup. By Lemma 4.11 if $H$ is normal in $G$ and $H / U$ is torsion-free, then so is $H / U^{G}$. On the other hand, if $H$ has finite index in $G$, then not more than $[G: H]$ of the $U^{g}$ are different. Consequently, if $H / U$ is elementary amenable, then so is $G / U^{G}$.

4.9. The main result. Now essentially everything is in place to prove the main (technical) theorem of this paper. We need one more lemma:

4.59. Lemma. Let $Q$ be a finite p-group and let $1 \rightarrow H \rightarrow G \rightarrow Q \rightarrow 1$ be an exact sequence of groups. Assume that $H$ is finitely generated and has enough torsion-free quotients. Specify an exhaustive family $\mathcal{A}_{H}^{\prime}$ of subgroups as in Definition 4.53, Fix $n \in \mathbb{N}$ and for every $U \in \mathcal{A}_{H}^{\prime}$ assume that $G / U^{G}$ contains a finite subgroup $E_{U} / U^{G}$ of order $n$.

Then there is a subgroup $Q_{0} \leq Q$ of order $n$ splitting back to $\hat{G}_{0}^{p} \leq \hat{G}^{p}$, where $G_{0}$ is defined by the exact sequence $1 \rightarrow H \rightarrow G_{0} \rightarrow Q_{0} \rightarrow 1$.

Proof. Since $H / U^{G}$ is torsion-free, the image of $E_{U} / U^{G}$ in $Q$ has order $n$, too. In particular, it follows that $n$ necessarily is a power of the prime number $p$. Let $a_{U}$ 
be the (finite) collection of subgroups of $Q$ of order $n$ which are images of finite subgroups of $G / U^{G}, U \in \mathcal{A}_{H}^{\prime}$.

If $U, V \in \mathcal{A}_{H}^{\prime}$, then each finite subgroup of $G /\left(U^{G} \cap V^{G}\right)$ maps isomorphically to a finite subgroup of $G / U^{G}$, since the kernel $U^{G} /\left(U^{G} \cap V^{G}\right)$ of the projection $G /\left(U^{G} \cap V^{G}\right) \rightarrow G / U^{G}$ is contained in the torsion-free group $H /(U \cap V)^{G}$, and similarly for $G / V^{G}$. In particular, $a_{U} \cap a_{V}$ contains $a_{U \cap V}$ and therefore is nonempty. Since the set of all subgroups of $Q$ is finite, there exists $Q_{0} \in \bigcap_{U \in \mathcal{A}_{H}^{\prime}} a_{U}$.

In other words, $Q_{0}$ splits back to every $G / U^{G}$ for $U \in \mathcal{A}_{H}^{\prime}$, and hence also to every quotient of $G / U^{G}$ which projects onto $G / H$. Among these quotients of $G / U^{G}$ $\left(U \in \mathcal{A}_{H}^{\prime}\right)$ we can find a cofinal set of finite $p$-group quotients of $G_{0}$. This follows as in the proof of Lemma 4.12, since $H$ has enough torsion-free quotients. By Lemma 4.52, $Q_{0}$ splits back to $\hat{G}_{0}^{p}$. Last observe that, by Lemma 4.12,

$$
1 \rightarrow \hat{H}^{p} \rightarrow \hat{G}_{0}^{p} \rightarrow Q_{0} \rightarrow 1 \quad \text { and } \quad 1 \rightarrow \hat{H}^{p} \rightarrow \hat{G}^{p} \rightarrow Q \rightarrow 1
$$

are exact, whence $\hat{G}_{0}^{p}$ is a subgroup of $\hat{G}^{p}$.

4.60. Theorem. Let $H$ be a discrete group with finite classifying space which has enough torsion-free quotients and which is cohomologically complete. Assume that $K H$ fulfills the strong Atiyah conjecture (for some subfield $K=\bar{K}$ of $\mathbb{C}$ ).

(1) Let

$$
1 \rightarrow H \rightarrow G \rightarrow Q \rightarrow 1
$$

be an exact sequence of groups, where $Q$ is a finite group. Then $K G$ fulfills the strong Atiyah conjecture.

(2) Moreover, if $G$ is torsion-free and $\mathcal{A}_{H}^{\prime}$ is an exhaustive family of subgroups of $H$ as in Definition 4.53, then we can find $U \in \mathcal{A}_{H}^{\prime}$ such that $G / U^{G}$ is torsion-free and elementary amenable.

4.61. Definition. Let $H$ be a group, $K$ a skew field and $D H$ a division ring which contains $K H$. We call $D H$ Hughes free if for every subgroup $U$ of $H$ and set of representatives $X \subset H$ of $H / U$ the set $X$ (considered a subset of $D H$ ) is linearly independent over the division closure $D U$ of $K U$ in $D H$.

4.62. Corollary. Assume that $H$ fulfills the assumptions of Theorem 4.60 and

$$
1 \rightarrow H \rightarrow G \rightarrow A \rightarrow 1
$$

is an extension with $A$ elementary amenable. If $\operatorname{lcm}(G)<\infty$, then $K G$ fulfills the strong Atiyah conjecture.

Assume that $k$ is a domain and $k * H$ embeds into a skew field $D_{H}$ such that the twisted action of $A$ on $k * H$ extends to $D_{H}$. If $G$ is torsion-free and $H$ has enough solvable-by-finite torsion-free quotients, then $k * G$ embeds into a skew field.

If we only require $H$ to have enough torsion-free quotients (not necessarily solvable-by-finite), but assume that $D_{H}$ is Hughes free, then $k * G$ also embeds into a skew field.

Proof of Corollary 4.62, It is a direct consequence of Proposition 2.6 and Theorem 4.60 that $K G$ fulfills the strong Atiyah conjecture.

For the statement about $k * G$, given a finite subgroup $E / H$ of $G / H$ we attempt to construct a suitable skew field extension $D_{E}$ of $D_{H}$ such that $D_{H} *[E / H]$ embeds into $D_{E}$. The result then follows from Lemma 2.5. 
Observe that $H$ is finitely generated (since it has finite classifying space) and hence by Lemma 4.55 an exhaustive family $\mathcal{A}_{H}^{\prime}$ exists. Using Theorem 4.60 with $E$ instead of $G$, choose $U \in \mathcal{A}_{H}^{\prime}$ such that $E / U^{E}$ is torsion-free. In view of Lemma 4.11. $E / U$ is elementary amenable.

Let $D_{U}$ be the division closure of $k * U^{E}$ in $D_{H}$. This is a skew field. The action of $E$ by conjugation on $D_{H} *[E / H]$ maps $k * U^{E}$ to itself and therefore maps the division closure $D_{U}$ to itself. Consequently, we can form $D_{U} *[E / U]$ and $D_{U} *[H / U]$. Since $H / U$ and $E / U$ are both torsion-free elementary amenable groups, and $D_{U}$ is a skew field, by Lemma 2.5 $D_{U} *[E / U]$ is an Ore domain with an Ore localization (of course a skew field) which we denote $D_{U, E}$. It has a sub-skew field $D_{U, H}$ which is the division closure of $D_{U} *[H / U]$, and of course at the same time is the Ore localization of this ring.

If $D_{H}$ is Hughes free, then any set of representatives for $H / U$ in $D_{H}$ is by definition linearly independent over $D_{U}$. This implies that $D_{U, H}$ is equal to $D_{H}$, therefore $D_{H} *[E / H]$ is a domain, since $D_{U, H} *[E / H]$ embeds into $D_{E}$.

However, we don't see a reason why $D_{U}$ should be equal to $D_{U, H}$ in general. Assume now that $H$ has enough solvable-by-finite torsion-free quotients and choose $\mathcal{A}_{H}^{\prime}$ to be solvable-by-finite exhaustive. In view of Lemma 4.11, and by replacing $U \in \mathcal{A}_{H}^{\prime}$ by $U^{G}$ we may assume that each $U \in \mathcal{A}_{H}^{\prime}$ is normal in $G$. Then we replace $D_{H}$ by a skew field built out of the (possibly many different) $D_{U, H}$.

Using a non-trivial ultra-filter $\mathbb{F}$ for the set $\mathcal{A}_{H}^{\prime}$ (i.e. an ultrafilter which contains all subsets with finite complement), we form a new skew field $D_{H}^{\prime \prime}$. Following [27, $\S 2.6]$ it consists of classes of all sequences $\left(d_{U}\right)_{U \in \mathcal{A}_{H}^{\prime}}$ with $d_{U} \in D_{U, H}$ (constructed as above), and

$$
\left(d_{U}\right)_{U \in \mathcal{A}_{H}^{\prime}}=\left(d_{U}^{\prime}\right)_{U \in \mathcal{A}_{H}^{\prime}}
$$

if and only if there is some $F \in \mathbb{F}$ such that $d_{U}=d_{U}^{\prime}$ for all $U \in F$. We embed $k * H$ in $D_{H}^{\prime \prime}$ via the map $x \mapsto(x, x, \ldots)$ and let $D_{H}^{\prime}$ be the division closure of $k * H$ in $D_{H}^{\prime \prime}$. In a similar manner we construct $D_{E}^{\prime}$ using $D_{U, E}$ instead of $D_{U, H}$. This skew field contains $D_{H}^{\prime}$. We should remark that a finite number of the $D_{U, E}$ not being a division ring (or not even being defined) does not affect $D_{E}^{\prime}$ being a division ring; in fact any finite number of the $D_{U, E}$ does not affect the isomorphism class of $D_{E}^{\prime}$. Our assumptions on $\mathcal{A}_{H}^{\prime}$ assure that $E / U$ is torsion-free for all but finitely many of the $U \in \mathcal{A}_{H}^{\prime}$.

Since $U$ is normal in $G$, the twisted action of $G / H$ on $k * H$ induces a twisted action on each $D_{U, H}$, hence on $D_{H}^{\prime \prime}$ and consequently on $D_{H}^{\prime}$. It follows that we may form the crossed product $D_{H}^{\prime} *[G / H]$.

Next we show that for each finite subgroup $E / H$ of $G / H$, the identity map on $k * E$ extends to a monomorphism from $D_{H}^{\prime} *[E / H]$ to $D_{E}^{\prime}$. We can describe $k * E$ as the free $k$-module with basis $\{\bar{e} \mid e \in E\}$ and multiplication satisfying $\overline{e_{1} e_{2}}=\lambda\left(e_{1}, e_{2}\right) \overline{e_{1} e_{2}}$ for some map $\lambda: E \times E \rightarrow k \backslash 0$. Let $\left\{x_{1}, \ldots, x_{n}\right\} \subset E$ be a set of coset representatives for $H$ and write $x_{i} x_{j}=h_{i j} x_{l(i, j)}$ where $h_{i j} \in H$ and $l(i, j) \in\{1, \ldots, n\}$. Then $D_{H}^{\prime} *[E / H]$ is the ring which is a free $D_{H}^{\prime}$-module with basis $\left\{\widehat{x_{1}}, \ldots \widehat{x_{n}}\right\}$, and multiplication satisfying $\widehat{x_{i}} \widehat{x_{j}}=\lambda\left(x_{i}, x_{j}\right) h_{i j} \widehat{x_{l(i, j)}}$. Now define $\theta: D_{H}^{\prime} *[E / H] \rightarrow D_{E}^{\prime}$ by

$$
\theta\left(\sum_{i=1}^{n} d_{i} \widehat{x_{i}}\right)=\sum_{i=1}^{n} d_{i} \overline{x_{i}}
$$


A routine check shows that $\theta$ is a ring homomorphism. Also it is easy to see that $\left\{\overline{x_{1}}, \ldots, \overline{x_{n}}\right\}$ is linearly independent over $D_{H}^{\prime}$ and it follows that $\theta$ is a monomorphism.

We deduce from the previous paragraph that $D_{H}^{\prime} *[E / H]$ is a domain for all finite subgroups $E / H$ of $G / H$. The result now follows by Lemma 2.5 .

4.63. Remark. Natural constructions of a skew field extension $D_{H}$ usually are Hughes free.

All examples of groups with finite classifying space known to us with enough torsion-free quotients actually have enough solvable-by-finite torsion-free quotients.

Consequently, the corresponding assumptions in Corollary 4.62 are not serious restrictions of generality.

Proof of Theorem 4.60. (1) For a prime number $p$ and a $p$-Sylow subgroup $S$ of $Q$, let $G_{S} \leq G$ be its inverse image in $G$. By Lemma 2.4 it suffices to establish the statement for every $K G_{S}$. To do this, we produce a subgroup $U$ of $H$, normal in $G_{S}$, such that $G_{S} / U$ is elementary amenable, $H / U$ is torsion-free and we have the divisibility relation $\operatorname{lcm}\left(G_{S} / U\right) \mid \operatorname{lcm}\left(G_{S}\right)$.

Since $H$ has a finite classifying space, it is torsion-free. Hence by [47, Proposition 4 and Lemma 3] it and its subgroup $U$ fulfill the strong Atiyah conjecture if and only if $D H$ and $D U$ are skew fields. In particular, we know that $D U$ is Artinian. We want to apply Proposition 2.6 to the extension

$$
1 \rightarrow U \rightarrow G_{S} \rightarrow G_{S} / U \rightarrow 1
$$

By Lemma 2.3. if $G_{E} / U \leq G_{S} / U$ is finite, then $G_{E} \leq G_{S}$ fulfills the assumptions of Proposition 2.6 with $L=\left|G_{E} / U\right|$, and therefore also with $L=\operatorname{lcm}\left(G_{S} / U\right)$. Since $\operatorname{lcm}\left(G_{S} / U\right)$ divides $\operatorname{lcm}\left(G_{S}\right)$, by Corollary 2.7 the strong Atiyah conjecture holds for $K G_{S}$.

It remains to find the subgroup $U$ with the required properties. Since $H$ is torsion-free and $G_{S} / H$ is a finite $p$-group, $\operatorname{lcm}\left(G_{S}\right)=p^{n}$ for some $n \geq 0$. Assume that every $G_{S} / U^{G_{S}}$ (defined in Definition 4.58) for $U \in \mathcal{A}_{H}$ contains a subgroup of order $p^{k}$. Note that each finite subgroup of each $G_{S} / U^{G_{S}}$ is a finite $p$-group, since $G_{S} / U^{G_{S}}$ contains the torsion-free subgroup $H / U^{G_{S}}$ with index a power of $p$. Therefore, it is sufficient to show that in this case $G_{S}$ itself contains a subgroup of order $p^{k}$. By Lemma 4.59 we can pass to subgroups $G_{0}$ of $G_{S}$ and $Q_{0}$ of $G_{S} / H$ such that $\left|Q_{0}\right|=p^{k}$ and the projection $\pi$ in

$$
1 \rightarrow \hat{H}^{p} \rightarrow \hat{G}_{0}^{p} \stackrel{\pi}{\rightarrow} Q_{0} \rightarrow 1
$$

splits. Now our important technical Theorem 4.27 applies and yields that $Q_{0}$ also splits back to $G_{0}$, hence gives a subgroup of order $p^{k}$ in $G_{S}$.

(2) Assume now that $G$ is torsion-free. The above argument implies that there is $U_{S}$ such that $G_{S} / U_{S}^{G_{S}}$ does not contain any non-trivial finite subgroup (the trivial group is now the biggest finite subgroup of $\left.G_{S} \leq G\right)$, i.e. $G_{S} / U_{S}^{G_{S}}$ is torsionfree. We can choose $U_{S} \in \mathcal{A}_{H}^{\prime}$ (since we can restrict ourselves to the use of $\mathcal{A}_{H}^{\prime}$ throughout).

Let $U$ be the intersection of all $U_{S}$ for all Sylow subgroups $S$ of $Q$. Since $G_{S} / U^{G}$ fits into the exact sequence

$$
1 \rightarrow U_{S}^{G} / U^{G} \rightarrow G_{S} / U^{G} \rightarrow G_{S} / U_{S}^{G} \rightarrow 1
$$


with both $G_{S} / U_{S}^{G}$ and $U_{S}^{G} / U^{G} \leq H / U^{G}$ torsion-free (by Lemma 4.11), $G_{S} / U^{G}$ is torsion-free.

If $G / U^{G}$ would contain a non-trivial torsion element, then by raising to an appropriate power we would have a $p$-torsion element $1 \neq x \in G / U^{G}$ for some prime number $p$. Since $H / U^{G}$ is torsion-free, $x$ would map to a $p$-torsion element in $G / H$, hence to a $p$-Sylow subgroup $S$ of $G / H$. Therefore, we could assume that $x$ is contained in $G_{S} / U^{G}$, which is torsion-free. This is a contradiction, hence $G / U^{G}$ itself is torsion-free. Since $H / U$ is elementary amenable, by Lemma 4.11 the same is true for $H / U^{G}$ and therefore also for its finite extensions $G / U^{G}$.

\section{Extensions of PURE BRAID GROUPS, ONE-RELATOR GROUPS AND LINK GROUPS}

The next task is to find examples to which Theorem 4.60 applies. In particular, we want to show that this is the case for the pure braid groups and generalizations thereof.

To do this, we will prove that certain types of extensions preserve the conditions of Theorem 4.60, and that we can obtain e.g. the braid groups using these constructions.

5.1. Extensions. We have introduced a number of properties of groups, in particular, to have enough torsion-free quotients, to be residually torsion-free nilpotent, and to be cohomologically complete. We now study when these properties are preserved by extensions of groups. This will be our main tool to produce interesting examples which have these properties.

5.1. Lemma. Let $1 \rightarrow H \rightarrow G \stackrel{p}{\rightarrow} Q \rightarrow 1$ be an exact sequence of groups. Assume that for each $m \in \mathbb{N}$ there is $n \in \mathbb{N}$ such that $\gamma_{n}(G) \cap H \subset \gamma_{m}(H)$.

(1) Assume that $H$ has enough torsion-free nilpotent quotients. If $Q$ has enough torsion-free nilpotent quotients, or enough torsion-free solvable quotients, or enough torsion-free solvable-by-finite quotients, respectively, then the same is true for $G$.

(2) Assume that $H$ is residually torsion-free nilpotent. If $Q$ is residually torsionfree nilpotent, and for each $n \in \mathbb{N}$ there is a factorization $Q \rightarrow Q / V \rightarrow$ $Q / \gamma_{n}(Q)$ with $Q / V$ torsion-free nilpotent, then $G$ is residually torsionfree nilpotent. If, in addition, for each $n \in \mathbb{N}$ there is a factorization $H \rightarrow H / W \rightarrow H / \gamma_{n}(H)$ with $H / W$ torsion-free nilpotent, then the corresponding statement is true for $G$.

Corresponding results hold if in the assumptions on $Q$ "nilpotent" is replaced by "solvable", or "solvable-by-finite", respectively, and the lower central series by the derived series or the appropriate series for solvable-byfinite quotients. Beware that, in the assumptions on $H$, we have to require nilpotent throughout.

Proof. We first prove (1). Therefore let $K \leq G$ be a normal subgroup with $G / K$ a finite $p$-group for some prime number $p$. Then we get an exact sequence

$$
1 \rightarrow H /(H \cap K) \rightarrow G / K \rightarrow Q / p(K) \rightarrow 1
$$

of finite $p$-groups. Let $V \leq H \cap K \leq H$ be a characteristic subgroup of $H$ such that $H / V$ is torsion-free nilpotent. Such a $V$ exists since $H$ has enough torsion-free nilpotent quotients. Since $H / V$ is nilpotent, there is an $m \in \mathbb{N}$ with $\gamma_{m}(H) \leq V$. 
Now choose $n \in \mathbb{N}$ with $\gamma_{n}(G) \cap H \leq \gamma_{m}(H) \leq V$, which exists by assumption. Choose $n$ in such a way that $\gamma_{n}(G) \leq K$ (this is possible since $G / K$ is nilpotent). Observe that $p\left(\gamma_{n}(G)\right)=\gamma_{n}(Q)$.

Since $Q$ has enough torsion-free nilpotent quotients, we find a characteristic subgroup $W$ of $Q$ such that $W \leq \gamma_{n}(Q)$, and $Q / W$ is torsion-free nilpotent. Assume that $\gamma_{n^{\prime}}(Q) \leq W$.

Now define the subgroup $U$ of $G$ as $V \cdot\left(\gamma_{n}(G) \cap p^{-1}(W)\right)$. Since $V \leq K$ and $\gamma_{n}(G) \leq K$, we get $U \leq K$.

Since $V$ is a characteristic subgroup of $H$, it is normal in $G$. Since $p$ is surjective, $p^{-1}(W)$ and $\gamma_{n}(G) \cap p^{-1}(W)$ are also normal in $G$, which implies that $U$ is a normal subgroup of $G$.

Moreover,

$$
\gamma_{n^{\prime}}(G) \subset \gamma_{n}(G) \cap p^{-1}(W) \subset V \cdot\left(\gamma_{n}(G) \cap p^{-1}(W)\right)=U,
$$

i.e. $G / U$ is nilpotent.

The map $G \rightarrow Q / W$ is surjective and has kernel $p^{-1}(W)$, which contains $U$. On the other hand, since $W \leq \gamma_{n}(Q)$,

$$
p^{-1}(W) \leq p^{-1}\left(\gamma_{n}(Q)\right)=H \cdot \gamma_{n}(G)
$$

i.e. $p^{-1}(W)=H \cdot\left(\gamma_{n}(G) \cap p^{-1}(W)\right)$. It follows that the induced map $G / U \rightarrow Q / W$ is defined and has kernel

$$
H \cdot\left(\gamma_{n}(G) \cap p^{-1}(W)\right) /\left(V \cdot\left(\gamma_{n}(G) \cap p^{-1}(W)\right)\right) \cong H /\left(H \cap\left(V \cdot\left(\gamma_{n}(G) \cap p^{-1}(W)\right)\right)\right) .
$$

Now $V \leq H \leq p^{-1}(W)$; therefore

$$
V \leq H \cap\left(V \cdot\left(\gamma_{n}(G) \cap p^{-1}(W)\right)\right)=V \cdot\left(\gamma_{n}(G) \cap H\right) \leq V \cdot \gamma_{m}(H) \leq V .
$$

This means that we get the exact sequence

$$
1 \rightarrow H / V \rightarrow G / U \rightarrow Q / W \rightarrow 1 .
$$

Since $H / V$ and $Q / W$ are both torsion-free the same is true for $G / U$. If $Q / W$ is only solvable, or solvable-by-finite, respectively, instead of nilpotent, (5.2) shows that the same is true for $G / U$.

Now we prove (2). For each $1 \neq g \in G$, we have to produce a projection $p_{g}: G \rightarrow R_{g}$ with $R_{g}$ torsion-free nilpotent and such that $p_{g}(g) \neq 1$. If $g$ is mapped to a non-trivial element of $Q$, this can be done because of the assumption that $Q$ is residually torsion-free nilpotent.

Assume therefore that $g \in H=\operatorname{ker}(p)$. Since $H$ is residually torsion-free nilpotent, there is a (without loss of generality characteristic) subgroup $V \leq H$ with $\gamma_{m}(H) \leq V$ and such that $H / V$ is torsion-free (and of course nilpotent), and such that $p(g) \neq 1$ for the projection $p: H \rightarrow H / V$. Now choose $n \in \mathbb{N}$ such that $\gamma_{n}(G) \cap H \leq \gamma_{m}(H) \leq V$, and a normal subgroup $W \leq Q$ with $W \leq \gamma_{n}(Q)$ and such that $Q / W$ is torsion-free and elementary amenable (such a $W$ exists by assumption). As in the proof of (1)] define the subgroup $U:=V \cdot\left(\gamma_{n}(G) \cap p^{-1}(W)\right)$. We get the exact sequence (5.2)

$$
1 \rightarrow H / V \rightarrow G / U \rightarrow Q / W \rightarrow 1
$$

and as before we conclude that $G / U$ is torsion-free nilpotent. If $Q / W$ is only solvable or solvable-by-finite, at least these properties are inherited by $G / U$.

Since $g$ maps to a non-trivial element in $H / V$, and the map $H / V \rightarrow G / U$ induced by the inclusion is injective, $g$ maps to a non-trivial element in $G / U$, as required. 
For the last remaining statement, fix $k \in \mathbb{N}$. We assume now that we find $W$ as above such that in addition $W \leq \gamma_{k}(H)$. We construct $U$ as before. (Observe that $k \leq m \leq n)$, and it only remains to show that $U \leq \gamma_{k}(G)$.

But $U \leq V \gamma_{n}(G) \leq \gamma_{k}(H) \gamma_{k}(G)=\gamma_{k}(G)$.

We need two different types of assumptions in Lemma [5.1] assumptions on the building blocks (which we obviously have to make to get corresponding results for the big group $G$ ) and assumptions on the extension itself. We now address the question of when the latter ones are satisfied.

5.3. Lemma. Let $1 \rightarrow H \rightarrow G \rightarrow Q \rightarrow 1$ be an exact sequence of groups such that $G$ acts unipotently on $H_{1}(H ; \mathbb{Z})$ as defined in Definition 4.14 . Assume that at least one of the following conditions is satisfied:

(1) $Q$ is nilpotent.

(2) The extension $1 \rightarrow H \rightarrow G \rightarrow Q \rightarrow 1$ is split, i.e. $G$ is a semi-direct product $G=H \rtimes Q$.

Then for each $m \in \mathbb{N}$ there is $n \in \mathbb{N}$ with $\gamma_{n}(G) \cap H \subset \gamma_{m}(H)$.

Proof. In the course of the proof we will have to use subgroups of iterated commutators. This can conveniently be dealt with using the bracketless notation as in [19. Given two subgroups $H$ and $K$ of a group $G$ we set $\gamma H K:=[H, K]$. We abbreviate $\gamma(\gamma H K) L=: \gamma^{2} H K L$. With this notation, $\gamma_{n}(G)=\gamma^{n-1} G^{n}$.

First observe that by definition $G$ acts unipotently on $H_{1}(H ; \mathbb{Z})=H / \gamma H^{2}$ if and only if there is $m \in \mathbb{Z}$ with

$$
\gamma^{m} H G^{m} \subset \gamma H^{2}=\gamma_{2}(H) .
$$

This follows since the action on $H / \gamma H^{2}$ is induced by conjugation. Since $\gamma^{m} H G^{m} \subset$ $\gamma_{m+1}(G) \cap H$, it is therefore necessary that $G$ acts unipotently on $H_{1}(H ; \mathbb{Z})$ for the conclusion of the lemma. [19, Lemma 7] says that Equation (5.4) implies that

$$
\gamma^{r m} H^{r} G^{r m-r+1} \subset \gamma^{r} H^{r+1}=\gamma_{r+1}(H) .
$$

Using this inductively, we also see that Equation (5.4) implies that for suitable $f(n) \in \mathbb{N}$

$$
\gamma^{f(n)} H G^{f(n)} \subset \gamma^{n} H^{n+1} .
$$

Assume first that $Q$ is nilpotent. This means that there is $n \in \mathbb{N}$ with $\gamma_{n}(G) \subset H$ (since it is mapped to $\gamma_{n}(Q)=\{1\}$ ). Combined with (5.6) this implies $\gamma_{n+f(m)}(G)$ $\subset \gamma_{m+1}(H)$, which is what we had to prove.

Assume now that the sequence $1 \rightarrow H \rightarrow G \rightarrow Q \rightarrow 1$ splits. With this assumption, we generalize a result of Falk and Randell [13, p. 85] who proved that $\gamma^{n}(G) \cap H=\gamma^{n}(H)$ for a semidirect product $G=H \rtimes Q$ if $Q$ acts trivially on $H_{1}(H ; \mathbb{Z})$.

Following Falk and Randell, let $p: G \rightarrow Q$ be the projection, and $j: Q \rightarrow G$ a split of $p$ (i.e. $p \circ j=\operatorname{id}_{Q}$ ). Using $j$, we consider $Q$ as a subgroup of $G$. Define

$$
\tau: G \rightarrow H: g \mapsto j\left(p\left(g^{-1}\right)\right) g=p\left(g^{-1}\right) g .
$$

Clearly $p(\tau(g))=1$ for each $g \in G$, i.e. $\tau$ indeed maps $G$ to $H$. For each $g \in G$, $g=h q$ with $h \in H$ and $q \in Q$ if and only if $h=\tau(g)$ and $q=p(g)$. In particular, if $g \in H$, then $g=\tau(g)$. 
The map $\tau$ is not a homomorphism, but satisfies

$$
\tau\left(g_{1} g_{2}\right)=p\left(g_{2}\right)^{-1} \tau\left(g_{1}\right) p\left(g_{2}\right) \cdot \tau\left(g_{2}\right)
$$

(for all these statements, compare [13, p. 83]).

We now claim that for each $m \in \mathbb{N}$ there is $n \in \mathbb{N}$ with

$$
\tau\left(\gamma_{n}(G)\right) \subset \gamma_{m}(H) .
$$

This implies the assertion, because $x \in \gamma_{n}(G) \cap H$ implies $x=\tau(x) \in \gamma_{m}(H)$.

We are not proving (5.8) directly, but rather prove by induction that for each $m \in \mathbb{N}$

$$
\tau\left(\gamma_{2^{m}}(G)\right) \subset \gamma^{m} H G^{m} .
$$

In view of the assumption that $\gamma^{m} H G^{m} \subset \gamma H^{2}$ and by (5.6) this implies (5.8).

We need the following lemma.

5.10. Lemma. If $H$ is a normal subgroup of $G$, then for each $m \in \mathbb{N}$

$$
\gamma^{m(m-1) / 2+1} G^{m(m-1) / 2+1} H \subset \gamma^{m} H G^{m} \text {. }
$$

Proof. This is the statement of [19, Theorem 2] applied to $H /\left(\gamma^{m} H G^{m}\right)$ and $G /\left(\gamma^{m} H G^{m}\right)$ inside $G /\left(\gamma^{m} H G^{m}\right)$.

The case $m=0$ of (5.9) is trivial.

For the induction step, assume $m \geq 1$ and choose $g_{1} \in \gamma_{2^{m}-1}(G) \subset \gamma_{2^{m-1}}(G)$ and $g_{2} \in G$, i.e. $\left[g_{1}, g_{2}\right]=g_{1}^{-1} g_{2}^{-1} g_{1} g_{2}$ is a typical element of $\gamma_{2^{m}}(G)$. Write $h_{1}=\tau\left(g_{1}\right)$, by induction $h_{1} \in \gamma^{m-1} H G^{m-1}$. Set $h_{2}=\tau\left(g_{2}\right)$. Similarly, set $q_{1}:=p\left(g_{1}\right) \in \gamma_{2^{m}-1}(Q) \subset \gamma_{2^{m}-1}(G), q_{2}:=p\left(g_{2}\right)$. We write $x^{y}=y^{-1} x y$. Then, following the calculation of [13, p. 85]

$$
\tau\left(\left[g_{1}, g_{2}\right]\right)=\left[q_{1}, q_{2}\right]^{-1}\left[q_{1}, h_{2}\right]^{h_{1}}\left[q_{1}, q_{2}\right]^{h_{2} h_{1}}\left[h_{1}, h_{2}\right]\left[h_{1}, q_{2}\right]^{h_{2}} .
$$

Using Lemma 5.10, $\left[q_{1}, h_{2}\right] \in \gamma^{2^{m}} G^{2^{m}} H \subset \gamma^{m} H G^{m}$, since $m(m-1) / 2+1 \leq 2^{m}$ for each $m \in \mathbb{N}, m \geq 1$. By induction, $\left[h_{1}, h_{2}\right],\left[h_{1}, q_{2}\right] \in \gamma^{m} H G^{m-1} G=\gamma^{m} H G^{m}$. Last, observe that $\left[\left[q_{1}, q_{2}\right], h_{2} h_{1}\right] \in \gamma^{2^{m}} G^{2^{m}} H \subset \gamma^{m} H G^{m}$, so $\left[q_{1}, q_{2}\right]$ commutes with $h_{2} h_{1}$ modulo $\gamma^{m} H G^{m}$. Consequently,

$$
\tau\left(\left[g_{1}, g_{2}\right]\right) \equiv\left[q_{1}, q_{2}\right]^{-1}\left[q_{1}, q_{2}\right]=1 \quad \bmod \gamma^{m} H G^{m}
$$

and so $\tau\left(\gamma_{2^{m}}(G)\right) \subset \gamma^{m} H G^{m}$.

This finishes the proof of Lemma 5.3

5.11. Remark. Given an extension $1 \rightarrow H \rightarrow G \rightarrow Q \rightarrow 1$, one can ask whether the condition that $G$ acts unipotently on $H_{1}(H ; \mathbb{Z})$ is sufficient to imply that for each fixed $m$, if $n$ is sufficiently large, then $\gamma_{n}(G) \cap H \subset \gamma_{m}(H)$. This is not the case in general. There are even counterexamples where $H$ is a central subgroup of $G$.

5.12. Lemma. Assume that $1=G_{0} \leq G_{1} \leq \cdots \leq G_{n}=G$ is a nested sequence of groups such that each $G_{i}$ is normal in $G$. Suppose that $G / G_{i}$ acts unipotently on $H_{1}\left(G_{i+1} / G_{i} ; \mathbb{Z}\right)$ for each $i=0, \ldots, n-1$. Then $G$ acts unipotently on $H_{1}\left(G_{i} ; \mathbb{Z}\right)$ for all $i$.

Proof. Induction reduces to the situation $1=G_{0} \leq G_{1} \leq G_{2} \leq G_{3}=G$, $G$ acts unipotently on $H_{1}\left(G_{1} ; \mathbb{Z}\right)$ and $G / G_{1}$ acts unipotently on $H_{1}\left(G_{2} / G_{1} ; \mathbb{Z}\right)$, and we 
have to show that $G$ acts unipotently on $H_{1}\left(G_{2} ; \mathbb{Z}\right)$. The exact sequence $1 \rightarrow$ $G_{1} \rightarrow G_{2} \rightarrow G_{2} / G_{1} \rightarrow 1$ yields an exact sequence

$$
H_{1}\left(G_{1} ; \mathbb{Z}\right) \rightarrow H_{1}\left(G_{2} ; \mathbb{Z}\right) \rightarrow H_{1}\left(G_{2} / G_{1} ; \mathbb{Z}\right) \rightarrow 0
$$

(observe that the first map is not injective in general). Since the group homomorphisms $G_{1} \rightarrow G_{2} \rightarrow G_{2} / G_{1}$ are compatible with the conjugation action by $G$, the same is true for the induced action in homology. Set $U:=H_{1}\left(G_{1} ; \mathbb{Z}\right)$, $V:=H_{1}\left(G_{2} ; \mathbb{Z}\right)$ and $W:=H_{1}\left(G_{2} / G_{1} ; \mathbb{Z}\right)$. We write $[U, G] \leq U$ for the subgroup of $U$ generated by the elements $g u-u$ for $g \in G, u \in U$ (using the $G$-action on $U$ induced by conjugation). As in the proof of Lemma [5.3, we use the notation $\gamma^{n} U G^{n}=\left[\gamma^{n-1} U G^{n-1}, G\right]$, with $\gamma U G=[U, G]$.

Since $G$ acts unipotently on $W, \gamma^{n_{2}} W G^{n_{2}}=0$ for some $n_{2} \in \mathbb{N}$. That means that $\gamma^{n_{2}} V G^{n_{2}}$ is mapped to zero in the exact sequence (5.13), i.e. lies in the image of $U$. Now $\gamma^{n_{1}} U G^{n_{1}}=0$ since $G$ acts unipotently on $U$ for suitable $n_{1} \in \mathbb{N}$. It follows that $\gamma^{n_{1}}\left(\gamma^{n_{2}} V G^{n_{2}}\right) G^{n_{1}}=\gamma^{n_{1}+n_{2}} V G^{n_{1}+n_{2}}=0$. In other words, $G$ acts unipotently on $H_{1}\left(G_{2} ; \mathbb{Z}\right)$.

5.14. Remark. Observe that, in the above lemma, even if $G / G_{i}$ acts trivially on $H_{1}\left(G_{i+1} / G_{i} ; \mathbb{Z}\right)$ for each $i$, we cannot conclude that $G_{i}$ acts trivially on $H_{1}\left(G_{i-1} ; \mathbb{Z}\right)$, but only that it acts unipotently.

5.15. Lemma. Assume that $N \leq G$ is a normal subgroup. Suppose $G$ acts unipotently on $H_{1}(N ; \mathbb{Z})$. Then $G$ acts unipotently on $H_{1}(N ; \mathbb{Z} / p)$ for each prime number $p$.

Proof. Since $H_{0}(N ; \mathbb{Z})=\mathbb{Z}$, by the universal coefficient theorem we have an isomorphism

$$
H_{1}(N ; \mathbb{Z} / p) \cong H_{1}(N ; \mathbb{Z}) \otimes_{\mathbb{Z}} \mathbb{Z} / p .
$$

This isomorphism is natural and therefore in particular compatible with the $G$ action. That $G$ acts unipotently on $H_{1}(N ; \mathbb{Z})$ means that there is an $n \in \mathbb{N}$ such that for $n$ arbitrary elements $g_{1}, \ldots, g_{n} \in G$ the operator $\left(g_{1}-1\right) \cdots\left(g_{n}-1\right)=0$ acting on $H_{1}(N ; \mathbb{Z})$. The universal coefficient theorem shows that the same is true for the action on $H_{1}(N ; \mathbb{Z} / p)$, i.e. the action of $G$ on $H_{1}(N ; \mathbb{Z} / p)$ is nilpotent, as well.

\subsection{The main class of groups.}

5.16. Definition. Let $\mathcal{F}$ be the class of groups which fulfill the following list of properties:

(1) $G$ has a finite classifying space $B G$.

(2) $G$ is cohomologically complete as defined in Definition 4.3,

(3) $G$ has enough nilpotent torsion-free quotients as in Definition 4.53 (for example, all the quotients in the lower central series $G / \gamma_{n}(G)$ are torsionfree).

Let $\mathcal{F}_{\infty}$ be the subclass of groups $G \in \mathcal{F}$ which are, in addition, residually nilpotent.

Observe that the definition of "enough nilpotent torsion-free quotients" immediately implies the following.

5.17. Remark. If $G \in \mathcal{F}_{\infty}$, then $G$ is residually torsion-free nilpotent. 
5.18. Lemma. If $H \in \mathcal{F}$, then $H$ is finitely generated, of finite cohomological dimension, torsion-free, and if $B$ is an $H$-module with finitely many elements, then $H^{n}(H, B)$ is finite for each $n$. Moreover, for each $k \in \mathbb{N}$ there is a normal subgroup $U_{k}$ of $H$ such that $H / U_{k}$ is torsion-free nilpotent and $U_{k} \leq \gamma_{k}(H)$.

Proof. Finiteness of $B H$ immediately implies that $H$ is torsion-free and the finiteness assertions. The existence of $U_{k}$ with the required properties is proved in Lemma 4.54.

5.19. Example. Every finitely generated free group $F_{n}$ belongs to $\mathcal{F}_{\infty}$.

Proof. Clearly $F_{n}$ has a finite classifying space. It is a classical result that $F_{n}$ is residually torsion-free nilpotent and that even $F_{n} / \gamma_{k}\left(F_{n}\right)$ is torsion-free for each $k$ (compare [41]). By Example 4.6. free groups are cohomologically complete. The assertion follows.

5.20. Proposition. Let $1 \rightarrow H \rightarrow G \rightarrow Q \rightarrow 1$ be a split exact sequence of groups (i.e. $G=H \rtimes Q$ ) and assume that $Q$ acts unipotently on $H_{1}(H ; \mathbb{Z})$.

If $H$ and $Q$ both belong to $\mathcal{F}$, then also $G \in \mathcal{F}$. If even $H, Q \in \mathcal{F}_{\infty}$, then $G \in \mathcal{F}_{\infty}$.

Proof. Since $H$ and $Q$ have finite classifying spaces, the same is true for $G$ by Lemma 4.30.

By Lemma 5.15, $Q$ acts unipotently on $H_{1}(H ; \mathbb{Z} / p)$ for each prime number $p$. Theorem 4.15 therefore implies that $G$ is cohomologically complete, the same being true for $H$ and $Q$ by assumption.

We assume that the sequence $1 \rightarrow H \rightarrow G \rightarrow Q \rightarrow 1$ is split exact. By Lemma 5.3 we can apply Lemma5.1 (1) to conclude that $G$ has enough torsion-free nilpotent quotients if $H$ and $Q$ both have the same property. In particular, if $H$ and $Q$ belong to $\mathcal{F}$, the same is true for $G$.

Because of Lemma 5.18, if $H$ and $Q$ belong to $\mathcal{F}_{\infty}$, i.e. are residually torsion-free nilpotent, we can apply Lemma $5.1(2)$ to conclude that $G$ is residually torsion-free nilpotent, as well, i.e. belongs also to $\mathcal{F}_{\infty}$.

5.21. Remark. Using Lemma 5.1 and Lemma 5.3, other variants of the statements of Proposition 5.20 can be proved. We leave this to the interested reader.

The next theorem shows why the classes $\mathcal{F}$ and $\mathcal{F}_{\infty}$ are important to us: if a group belongs to them, then the Atiyah conjecture is inherited by extensions with elementary amenable quotient.

5.22. Theorem. Assume that $H \in \mathcal{F}$ and $H$ fulfills the strong Atiyah conjecture over $K H$, where $K=\bar{K}$ is a subfield of $\mathbb{C}$. For example, assume that $H \in \mathcal{F}_{\infty}$ and $K=\overline{\mathbb{Q}}$, where $\overline{\mathbb{Q}}$ is the algebraic closure of $\mathbb{Q}$ in $\mathbb{C}$. Let $G$ be an extension of $H$ with $\operatorname{lcm}(G)<\infty$ and such that $G / H$ is elementary amenable. Then $G$ fulfills the strong Atiyah conjecture over $K G$.

If the identity map of $K H$ extends to an isomorphism $K H_{\Sigma(H)} \rightarrow D H$, then the identity map of $K G$ extends to an isomorphism $K G_{\Sigma(G)} \rightarrow D G$.

Let $\mathcal{A}_{H}^{\prime}$ be a nilpotent exhaustive set of subgroups of $H$. If, in addition, $G$ is torsion-free and $G / H$ is finite, then for $n \in \mathbb{N}$ sufficiently large we find $U_{n} \in \mathcal{A}_{H}^{\prime}$ with $U_{n} \leq \gamma_{n}(H)$ such that $G / U_{n}$ is torsion-free (and of course virtually nilpotent). 
If, again with $G$ torsion-free, we have a domain $k$ and a crossed product $k * G$ such that the subring $k * H$ embeds into a skew field $D_{H}$ and the twisted action of $Q$ on $k * H$ extends to $D_{H}$, then $k * G$ embeds into a skew field.

Proof. If $G \in \mathcal{F}_{\infty}$, then $\overline{\mathbb{Q}} G$ satisfies the strong Atiyah conjecture by Corollary 3.3 . The other statements are immediate consequences of Lemma 5.18, Theorem 4.60 and Corollary 4.62. Here we use the fact that all groups in $\mathcal{A}_{H}^{\prime}$ are characteristic. In the first instance, Theorem 4.60 produces only one $U \in \mathcal{A}_{H}^{\prime}$ with $G / U$ torsionfree. Obviously $U=: U_{n_{0}} \leq \gamma_{n_{0}}(H)$ for some $n_{0} \in \mathbb{N}$. For $n>n_{0}$, choose $V_{n} \in \mathcal{A}_{H}^{\prime}$ with $V_{n} \leq \gamma_{n}(H)$. Set $U_{n}:=U \cap V_{n} \in \mathcal{A}_{H}^{\prime}$. The exact sequence $1 \rightarrow U / U_{n} \rightarrow G / U_{n} \rightarrow G / U \rightarrow 1$ shows that $G / U_{n}$ is torsion-free since $G / U$ and $U / U_{n} \leq H / U_{n}$ are torsion-free. By Lemma 4.11, $G / U_{n}$ is virtually nilpotent.

The following example shows that the additional assumptions in Theorem 5.22 are often satisfied.

5.23. Example. Suppose $H$ is poly-orderable, i.e. admits a finite normal series with orderable factors. Assume that $G$ is a torsion-free extension of $H$ and $G / H$ is elementary amenable. If $k * G$ is a crossed product with a skew field $k$, then the crossed product $k * H$ embeds into a canonically defined skew field $D_{G}$, which comes with a (unique) twisted action of $G / H$ extending the twisted action on $k * H$. Furthermore, if $H$ is poly-free, the identity map of $K H$ extends to an isomorphism $K H_{\Sigma(H)} \rightarrow D H$.

Remember that orderable means two-sided orderable, and that every residually torsion-free nilpotent group, in particular every finitely generated free group, is orderable 4, Theorems 2.1.1 and 3.1.5]. We want to remark that all the groups in $\mathcal{F}_{\infty}$ are residually torsion-free nilpotent and therefore poly-orderable. Many, like the pure braid groups, are also poly-free.

Proof. The second statement follows by applying [36. Lemma 12.5] to each of the finitely many free extensions in the construction of $H$ as a poly-free group, using the fact that poly-free groups fulfill the strong Atiyah conjecture (we note that 36 , Lemma 12.5] remains true when $\mathbb{C}$ is replaced by an arbitrary subfield $K$ of $\mathbb{C}$ which is closed under complex conjugation).

For the first statement, we know from $[23, \S 1]$ that $k * H$ has a free division ring of fractions in the sense of Hughes [22, §2]. This means in particular that any automorphism of $k * H$ which preserves the monomials of $k * H$ (i.e. sends an element of the form $a h$ with $a \in k$ and $h \in H$ to another such element) extends uniquely to an automorphism of $D_{H}$. Thus we get the required (unique) twisted action of $G / H$ on $D_{H}$ extending the action on $k * H$.

We continue with some abstract results which show how the methods we have developed so far give information for certain groups beyond the class $\mathcal{F}$.

5.24. Lemma. Let $G_{1}, \ldots, G_{N}$ be torsion-free solvable groups and

$$
G:=G_{1} * \cdots * G_{N}
$$

their free product. Every projection $G \rightarrow Q$ with $Q$ solvable has a factorization $G \rightarrow Q_{1} \rightarrow Q$ where $Q_{1}$ is torsion-free solvable.

Proof. By [18, Lemma 5.5] we have an exact sequence

$$
1 \rightarrow F \rightarrow G_{1} * \cdots * G_{N} \rightarrow G_{1} \times \cdots \times G_{N} \rightarrow 1,
$$


where $F$ is a free group. Let $U$ be the kernel of the projection $G \rightarrow Q$. Since $Q$ is solvable $F /(F \cap U)$ also is solvable. Therefore, $F \cap U$ contains the derived series subgroup $F^{(k)}$ for a suitable $k \in \mathbb{N}$. Since every subgroup of a free group is free, the abelianization $F^{(k)} / F^{(k-1)}$ of every derived series subgroup of $F$ is free abelian and in particular torsion free. By induction, using the exact sequence $1 \rightarrow$ $F^{(k-1)} / F^{(k)} \rightarrow F / F^{(k)} \rightarrow F / F^{(k-1)} \rightarrow 1, F / F^{(k)}$ is also torsion-free. Moreover, since $F^{(k)}$ is a characteristic subgroup of $F$, it is normal in $G$. Since $G_{1}, \ldots, G_{N}$ are solvable, for sufficiently large $k$ we get an extension

$$
1 \rightarrow F / F^{(k)} \rightarrow G / F^{(k)} \rightarrow G_{1} \times \cdots \times G_{N} \rightarrow 1
$$

of $G / F^{(k)}$ by torsion-free solvable groups, therefore $G / F^{(k)}$ itself is torsion-free solvable, and by the choice of $F^{(k)}$ it maps onto $G / U=Q$.

5.25. Proposition. Assume that $N \in \mathbb{N}$ and that $G_{1}, G_{2}, \ldots, G_{N} \in \mathcal{F}$.

(1) Then the free product $G:=G_{1} * G_{2} * \cdots * G_{N}$ has enough solvable torsion-free quotients.

(2) If, more restrictively, $G$ has enough nilpotent torsion-free quotients, then $G$ belongs to $\mathcal{F}$.

(3) If $G_{i}$ is residually torsion-free solvable for each $i$ (e.g. if $G_{i} \in \mathcal{F}_{\infty}$ ), then the same is true for $G$.

Proof. For (2) observe that the classifying space of $G$ is the one-point union of the classifying spaces of $G_{j}$ for $j=1, \ldots, N$, and therefore is compact. By Proposition 4.25. $G$ is cohomologically complete. If $G$ has enough nilpotent torsion-free quotients, then it follows that $G$ belongs to $\mathcal{F}$.

To prove (3), remember that the property of being torsion-free solvable is a root property in the sense of [18, and free groups are residually torsion-free solvable, even residually torsion-free nilpotent (compare [41]). Therefore, by [18, Theorem 4.1], $G$ is residually torsion-free solvable if the same is true for all the $G_{i}$.

To establish (1) given a normal subgroup $U$ of $G$, if we set $U_{j}:=G_{j} \cap U$, then $G / U$ is a quotient of $\left(G_{1} / U_{1}\right) * \cdots *\left(G_{N} / U_{N}\right)$. If the index of $U$ in $G$ is a power of $p$, the same is true for the index of $U_{j}$ in $G_{j}$ for $j=1, \ldots, N$. Be definition of the class $\mathcal{F}$, we find that $V_{j} \leq U_{j}$ normal in $G_{j}$ such that $G_{j} / V_{j}$ is torsion-free nilpotent and hence torsion-free solvable. By Lemma 5.24 applied to the solvable (since it is a finite $p$-group) quotient $G / U$ of $\left(G_{1} / V_{1}\right) * \cdots *\left(G_{N} / V_{N}\right)$ we find a factorization $\left(G_{1} / V_{1}\right) * \cdots *\left(G_{N} / V_{N}\right) \rightarrow Q_{1} \rightarrow G / U$ where $Q_{1}$ is torsion-free solvable. It follows from Lemma 4.55 that $G$ has enough solvable-by-finite torsion-free quotients.

5.26. Proposition. Let $G_{1}, \ldots, G_{N}$ be groups which belong to $\mathcal{F}_{\infty}$. Assume that $Q$ has a finite classifying space, is cohomologically complete, is residually torsion-free solvable-by-finite and has enough solvable-by-finite torsion-free quotients. Define

$$
G:=G_{1} \rtimes\left(G_{2} \rtimes\left(\cdots\left(G_{N} \rtimes Q\right)\right)\right),
$$

and assume that in each semidirect product the quotient acts unipotently on the first homology of the kernel. Then $G$ has a finite classifying space, is residually torsion-free solvable-by-finite, has enough torsion-free solvable-by-finite quotients, and is cohomologically complete. If there is an exact sequence

$$
1 \rightarrow G \rightarrow H \rightarrow A \rightarrow 1
$$

with $A$ elementary amenable and $\operatorname{lcm}(H)<\infty$, then $\overline{\mathbb{Q}} H$ fulfills the strong Atiyah conjecture. 
Proof. By an iterative application of Lemma 4.30. $G$ has a finite classifying space, and by Lemma 5.3 and Lemma $5.1, G$ has enough solvable-by-finite torsion-free quotients and is residually torsion-free solvable-by-finite (observe that having enough torsion-free solvable-by-finite quotients implies the factorization property for the lower central series quotients as required in Lemma 5.1 (2) because of Lemma 4.54). Theorem 4.15implies that $G$ is cohomologically complete. The last assertion follows from Corollary 4.62 and Corollary 3.3 .

5.27. Example. In Proposition 5.26, $Q=Q_{1} * \cdots * Q_{m}$ can be a free product of finitely many groups $Q_{i} \in \mathcal{F}_{\infty}$. By Proposition 5.25, $Q$ has enough solvable torsionfree quotients and is residually torsion-free solvable, and by Proposition $4.25 Q$ is cohomologically complete. Its classifying space is the one-point union of the $B Q_{i}$, $i=1, \ldots, m$, and therefore is finite.

In the next subsections we describe certain interesting classes of groups which belong to $\mathcal{F}$ or even to $\mathcal{F}_{\infty}$.

\subsection{One-relator groups.}

5.28. Definition. A one-relator group $G$ is called primitive if it is finitely generated and if it has a presentation $G=\left\langle x_{1}, \ldots, x_{d} \mid r\right\rangle$ such that the element $r$ in the free group $F$ generated by $x_{1}, \ldots, x_{d}$ is contained in $\gamma_{n}(F)$ but not in $\gamma_{n+1}(F)$ and the image of $r$ in $\gamma_{n}(F) / \gamma_{n+1}(F)$ is not a proper power.

We adopt the convention that finitely generated free groups are primitive onerelator groups.

5.29. Example. The following finitely generated one-relator groups are primitive:

- one-relator groups where the least common multiple of the exponent-sums for the different generators in the relator is one,

- fundamental groups of compact orientable two-dimensional surfaces (with or without boundary).

5.30. Proposition. Let $G$ be a primitive one-relator group. Then $G$ belongs to $\mathcal{F}$.

Proof. By [31, Exemple (2), p. 144] such a group is cohomologically complete. The main theorem of 32 says in particular that $G / \gamma_{n}(G)$ is torsion-free for each $n \in \mathbb{N}$. The fact that $G$ is primitive implies that the relator $r$ is not a proper power. By [40, $G$ has cohomological dimension 2, more precisely, the presentation complex of $\left\langle x_{1}, \ldots, x_{d} \mid r\right\rangle$ with $d$ 1-cells and one 2 -cell is a model for $B G$, i.e. $B G$ is finite.

5.31. Remark. In light of Theorem 5.22 and Proposition 5.30 it is of course important to know which one-relator groups satisfy the Atiyah conjecture. Examples for this are residually torsion-free solvable one-relator groups. Unfortunately, we don't know whether all one-relator groups, or at least all torsion-free one-relator groups, satisfy the Atiyah conjecture.

\subsection{Link groups.}

5.32. Definition. We denote the fundamental group $G$ of the complement $M$ of a tubular neighborhood $\nu(L)$ of a tame link $L$ with $d$ components in $S^{3}$ as a link group with $d$ components. We define the linking diagram to be the edge-labeled graph whose vertices are the components of the link, and such that any pair of vertices is joined by exactly one edge. Each edge is labeled with the linking number of the two link components involved. 
We say the link group $G$ is primitive if for each prime number $p$ there is a spanning subtree of the linking diagram such that none of the labels of the edges of this subtree is congruent to 0 modulo $p$.

Observe that in particular every knot group (i.e. a link group with only one component) is primitive.

5.33. Definition. A compact orientable 3-manifold (possibly with boundary) is called irreducible if every embedded two-sphere bounds an embedded three-disc. It is called Haken if it is irreducible and if there is a properly embedded two-sided surface different from a two-sphere such that the induced map of fundamental groups is injective.

5.34. Proposition. Assume that $G$ is a primitive link group with $d$ components. Then $G$ has a finite classifying space and $G / \gamma_{n}(G)$ is torsion-free for each $n \in \mathbb{N}$. Moreover, $G \in \mathcal{F}$.

Proof. Let $M \subset S^{3}$ be the link-complement such that $G=\pi_{1}(M)$. Then $M$ is an orientable manifold with non-empty boundary, and each boundary component is a torus. Moreover, primitivity in particular implies that the link is non-split, i.e. if we have a two-sphere embedded into the complement of the link in $S^{3}$, then by Alexander's theorem the link will intersect only one of the two discs into which this two-sphere splits $S^{3}$ (otherwise the linking graph would contain two non-empty parts, connected only by edges labeled with zero). This also shows that each twosphere embedded into the complement of the link bounds a three-disc embedded into the complement (the one of the above three-discs which does not intersect the link). By definition, this means that $M$ is irreducible.

Since the boundary of $M$ contains a surface which is not a two-disc, $M$ is a Haken manifold by [20, Lemmas 6.6 and 6.7]. By 26, Remark III.19] the finite CW-complex $M$ is the classifying space of $G$ (this is also directly a consequence of [42, Theorem 27.1]). By [33, Theorems 2 and 1] $G / \gamma_{n}(G)$ is torsion-free for each $n \in \mathbb{N}$.

In [30] we show that $G$ is cohomologically $p$-complete for every prime number $p$. This finishes the proof.

The following proposition is a special case of Proposition 5.34. We single it out here because we can give an independent and more elementary proof of the key property, namely cohomological completeness.

5.35. Proposition. Let $G$ be a knot group, i.e. the fundamental group of the complement of a tame knot in $S^{3}$, or let $G$ be a primitive link group with two components. Then $G \in \mathcal{F}$.

Proof. Above, we have given a proof of the fact that $G$ has a finite classifying space, namely the knot or link complement $S^{3}-\nu(L)$. Let $d \in\{1,2\}$ be the number of components of $L$.

Let $h: G \rightarrow G^{a b}=H_{1}(G, \mathbb{Z})$ be the projection of $G$ onto its abelianization.

We claim that $G^{a b}$ is isomorphic to $\mathbb{Z}^{d}$ and that $h$ induces an isomorphism in homology and cohomology (with integral coefficients). The assertion then follows from Proposition 5.37 and Example 5.39 .

It remains to prove the claim. 
A classical computation in knot theory, using Alexander duality, shows that $H_{n}(G, \mathbb{Z})$ and $H^{n}(G, \mathbb{Z})$ are free abelian for each $n$, and

$$
\operatorname{rank} H^{n}(G, \mathbb{Z})= \begin{cases}1, & \text { if } n=0 \\ d, & \text { if } n=1 \\ d-1, & \text { if } n=2 \\ 0, & \text { if } n>2\end{cases}
$$

Hence for trivial reasons (since $G^{a b}$ is free abelian) $H^{n}(h)$ is an isomorphism except possibly for $n=2$ and $d=2$. In this case $G^{a b}=\mathbb{Z}^{2}$ and the generator of $H^{2}\left(\mathbb{Z}^{2}, \mathbb{Z}\right)$ is the cup product of the two generators of $H^{1}\left(\mathbb{Z}^{2}, \mathbb{Z}\right)$. Alexander duality and the definition of the linking number implies that the cup product of the two generators of $H^{1}(G, \mathbb{Z})$ is the linking number times the generator of $H^{2}(G, \mathbb{Z})$. By assumption, this linking number is \pm 1 , so that $G \rightarrow G^{a b}$ also induces an isomorphism on $H^{2}$. Using the universal coefficient theorem to see that $H_{n}(h)$ is also an isomorphism for all $n$, this concludes the proof.

5.36. Corollary. Let $H$ be either a primitive one-relator group (compare Remark 5.31) or a primitive link group, and assume that $H$ is residually torsion-free solvable.

If there is an exact sequence $1 \rightarrow H \rightarrow G \rightarrow A \rightarrow 1$ with $A$ elementary amenable and $\operatorname{lcm}(G)<\infty$, then the strong Atiyah conjecture is true for $\overline{\mathbb{Q}} G$.

Proof. By Proposition 5.30 or by Proposition 5.34, $H \in \mathcal{F}$. It therefore follows in both cases by Corollary 3.3 that $\overline{\mathbb{Q}} H$ satisfies the strong Atiyah conjecture.

\subsection{Homology-invariance.}

5.37. Proposition. Assume that $G \in \mathcal{F}$, and that $H_{1}, H_{2}$ are groups with finite classifying spaces and $f_{1}: H_{1} \rightarrow G, f_{2}: G \rightarrow H_{2}$ are group homomorphisms which induce isomorphisms on homology with integral coefficients. Then $H_{1}$ and $H_{2}$ both belong to $\mathcal{F}$, and $f_{1}$ as well as $f_{2}$ induce an isomorphism between the pro-p completions (for every prime number $p$ ).

Proof. By [51, Theorem 3.4], $f_{1}$ or $f_{2}$ induce isomorphisms between the lower central series quotients $G / \gamma_{n}(G)$ and $H_{1} / \gamma_{n}\left(H_{1}\right)$ or $H_{2} / \gamma_{n}\left(H_{2}\right)$, respectively, for every $n \in \mathbb{N}$. This implies that the maps induce isomorphisms between the inverse systems of nilpotent quotients of $G, H_{1}$ and $H_{2}$. Hence, by Lemma 4.55 since $G$ has enough torsion-free nilpotent quotients, the same is true for $H_{1}$ and for $H_{2}$.

Moreover, in particular the finite $p$-group quotients of $G, H_{1}$ and $H_{2}$ coincide (since they are nilpotent). This means we get the following commuting diagram:

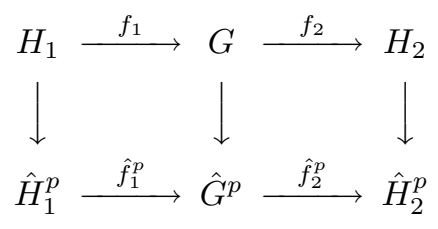


and $\hat{f}_{1}^{p}$ as well as $\hat{f}_{2}^{p}$ are isomorphisms. Taking cohomology with $\mathbb{Z} / p$-coefficients, we obtain

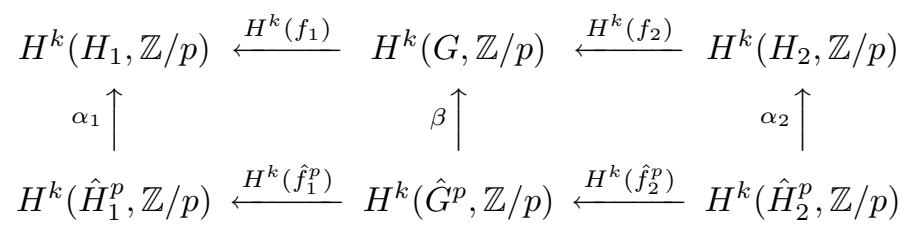

where now not only $H^{k}\left(\hat{f}_{1}^{p}\right)$ and $H^{k}\left(\hat{f}_{2}^{p}\right)$, but by assumption, using the universal coefficient theorem for cohomology, also $\beta, H^{k}\left(f_{1}\right)$ and $H^{k}\left(f_{2}\right)$ are isomorphisms. It follows that $\alpha_{1}$ and $\alpha_{2}$ are isomorphisms, too. This implies that $H_{1}$ and $H_{2}$ are cohomologically complete and concludes the proof.

5.38. Definition. A discrete group $G$ is called acyclic if $H_{k}(G, \mathbb{Z})=0$ for $k>0$.

5.39. Example. It is well known that finitely generated free abelian groups belong to $\mathcal{F}$. Cohomological completeness, which is the only non-obvious point, follows from Proposition 5.20 .

Let $G$ be a group with finite classifying space and such that $H_{1}(G, \mathbb{Z})$ is free abelian. Assume that the projection $G \rightarrow G /[G, G]=H_{1}(G, \mathbb{Z})$ is a homology isomorphism, i.e. $H_{k}(G, \mathbb{Z}) \rightarrow H_{k}(G /[G, G], \mathbb{Z})$ is an isomorphism for every $k \in \mathbb{N}_{0}$. It follows from Proposition 5.37 that $G \in \mathcal{F}$. Observe that this follows in particular if $G$ is acyclic or if $H_{*}(G, \mathbb{Z}) \cong H_{*}(\mathbb{Z}, \mathbb{Z})$ (as abstract abelian groups).

We will see in Proposition 5.35 that knot groups and certain link groups provide examples where the projection to the abelianization is a homology isomorphism.

Acyclic groups with finite classifying space are not hard to come by. They play an important role in asphericalization processes: given any finite simplicial complex $X$, we can use any acyclic group with finite classifying space as a building block to construct a new finite simplicial complex $B L X$ which has the same cohomology as $X$ and is aspherical (i.e. which is the classifying space of its fundamental group $\left.L X:=\pi_{1}(B L X)\right)$. For all this, compare 3 .

Fix an acyclic group which has a finite classifying space. Following [3, Section 9], this gives rise to an asphericalization procedure, a functor $B L$ from finite simplicial complexes to aspherical finite simplicial complexes (more precisely, to $X$ we assign a map $B L X \rightarrow X$ which induces a surjection on $\pi_{1}$ and an isomorphism in integral homology).

If we take any acyclic finite simplicial complex $X$ and apply the asphericalization construction, then we obtain a new acyclic group $L X=\pi_{1}(B L X)$.

As a preparation to get non-acyclic examples, let $X$ and $Y$ be two spaces whose integral cohomology ring in both cases is isomorphic to the cohomology ring of $\mathbb{Z}^{n}$, i.e. is a symmetric algebra over $\mathbb{Z}$ on $n$ generators which belong to $H^{1}$. Let $f: Y \rightarrow X$ be a map which induces an isomorphism $H^{1}(f): H^{1}(X, \mathbb{Z}) \rightarrow H^{1}(Y, \mathbb{Z})$. Then $f$ is a homology isomorphism, because an algebra map like $H^{*}(f)$ which is a bijection between the generators of two symmetric algebras is an isomorphism, and then we can apply the universal coefficient theorem.

Assume next that we apply the asphericalization procedure described above to a finite simplicial complex $X$ whose integral cohomology ring is isomorphic to the cohomology ring of the free abelian group $\mathbb{Z}^{n}$. We get a homology isomorphism $B L X \rightarrow X$, in particular $H_{1}(B L X, \mathbb{Z})=\mathbb{Z}^{n}$ and $H^{*}(B L X, \mathbb{Z})$ is the cohomology ring of $\mathbb{Z}^{n}$. The projection $\pi: L X \rightarrow H_{1}(L X, \mathbb{Z})=L X /[L X, L X]=\mathbb{Z}^{n}$ induces by 
duality an isomorphism on the first cohomology with integral coefficients. Therefore, it is a homology isomorphism. By Proposition 5.37, $L X$ belongs to $\mathcal{F}$.

Note that the conditions on the cohomology ring structure are trivially fulfilled if $H_{*}(X, \mathbb{Z}) \cong H_{*}(\mathbb{Z}, \mathbb{Z})$ (as abelian groups).

\subsection{Braid groups.}

5.40. Theorem. Assume that $H$ is the fundamental group of a (complement of a) fiber-type arrangement. Then, $H$ belongs to $\mathcal{F}_{\infty}$. In particular, this is the case for Artin's classical pure braid groups and, more generally, for the generalized pure braid groups associated to the Coxeter groups $A_{l}, C_{l}, G_{2}$ and $I_{2}(p)$ 14, Theorem 3.1].

Proof. Falk and Randell construct [13, Proposition 2.5] $G$ as an iterated semidirect product with trivial action on $H_{1}$ of the kernel, starting with a finitely generated free group. By Example 5.19 and Proposition 5.20, $G \in \mathcal{F}_{\infty}$ (it is already proved in [14, Theorem 2.6] that $G$ is residually torsion-free nilpotent).

5.41. Corollary. Assume that $H$ is one of the groups of Theorem 5.40 e.g. a classical Artin pure braid group $P_{n}$. Then every finite extension $G$ of $H$ fulfills the strong Atiyah conjecture over $\overline{\mathbb{Q}}$. Moreover, if such an extension is torsion-free, then it is residually (torsion-free virtually nilpotent), i.e. it has plenty of non-trivial torsionfree quotients.

This applies in particular to Artin's classical full braid groups $B_{n}$ (as well as the full braid groups associated to $C_{n}, G_{2}$ and $I_{2}(p)$ ).

Specifically, for each classical braid group $B_{n}(n \in \mathbb{N}$ arbitrary) the quotients $B_{n} / \gamma_{N}\left(P_{n}\right)$ are torsion-free for all $N \geq N(n)$ sufficiently large, and the intersection of the $\gamma_{N}\left(P_{n}\right)$ is trivial. This also implies that the commutator subgroup $B_{n}^{\prime}$ has non-trivial torsion-free quotients.

In view of Example 5.23, the additional statements about localization and crossed products in Theorem 5.22 apply. In particular, if we have an exact sequence $1 \rightarrow$ $H \rightarrow G \rightarrow A \rightarrow 1$ where $A$ is elementary amenable, and $\operatorname{lcm}(G)<\infty$, then the strong Atiyah conjecture is true for $\overline{\mathbb{Q}} G$. If $G$ is torsion-free and $k * G$ is a crossed product with a skew field $k$, then $k * G$ embeds into a skew field.

Proof. By Theorem 5.40, this follows from Theorem 5.22 .

5.42. Remark. It was a question of Lin [34, 0.9 e,f, Remark 7.3] whether the braid groups $B_{n}$ have non-trivial non-abelian torsion-free quotients (actually, he conjectures this not to be the case for $n>4$ ). This question is also popularized as question B6 on the group theory server http://www.grouptheory.info. The question is answered with yes for $n \leq 6$ in [24] with an explicit torsion-free quotient. Humphries' computations are rather involved. In the course of them he makes use of computer algebra programs. He has explicit candidates for torsion-free quotients of $B_{n}$ for all $n$. Humphries proves that the kernel $K_{n}$ of his projection contains $\gamma_{n}\left(P_{n}\right)$ and, for $n \leq 6$, is contained in $P_{n}$. Explicit calculations of the ranks of the nilpotent abelian quotients of $P_{n} / K_{n}$ show that in general $K_{n} \neq \gamma_{k}\left(P_{n}\right)$ for any $k \in \mathbb{N}$.

As a by-product of our approach, we get a torsion-free quotient as asked for by Lin for each $B_{n}$ (the only drawback is that we only give an existence proof, but without further work we can't specify the integers $N$ for which $G_{n} / \gamma_{N}\left(P_{n}\right)$ is torsion-free). 


\section{REFERENCES}

[1] Alejandro Adem and R. James Milgram. Cohomology of finite groups. Springer-Verlag, Berlin, 1994. MR1317096 (96f:20082)

[2] M. F. Atiyah. Elliptic operators, discrete groups and von Neumann algebras. In Colloque "Analyse et Topologie" en l'Honneur de Henri Cartan (Orsay, 1974), pages 43-72. Astérisque, No. 32-33. Soc. Math. France, Paris, 1976. MR0420729 (54:8741)

[3] G. Baumslag, E. Dyer, and A. Heller. The topology of discrete groups. J. Pure Appl. Algebra, 16(1):1-47, 1980. MR549702 (81i:55012)

[4] Roberta Botto Mura and Akbar Rhemtulla. Orderable groups. Marcel Dekker Inc., New York, 1977. Lecture Notes in Pure and Applied Mathematics, Vol. 27. MR 0491396 (58:10652)

[5] Nicolas Bourbaki. Commutative algebra. Chapters 1-7. Springer-Verlag, Berlin, 1989. Translated from the French, Reprint of the 1972 edition. MR0979760 (90a:13001)

[6] Clemens Bratzler. $L^{2}$-Betti Zahlen und Faserungen. Diplomarbeit, Universität Mainz, 1997. http://wwwmath.uni-muenster.de/u/lueck/group/bratzler.dvi.

[7] Kenneth S. Brown. Cohomology of groups. Springer-Verlag, New York, 1982. MR672956 (83k:20002)

[8] P. M. Cohn. Free rings and their relations. Academic Press Inc. [Harcourt Brace Jovanovich Publishers], London, second edition, 1985. MR800091 (87e:16006)

[9] Warren Dicks and Thomas Schick. The spectral measure of certain elements of the complex group ring of a wreath product. Geom. Dedicata, 93:121-137, 2002. MR1934693(2003i:20005)

[10] Jozef Dodziuk. De Rham-Hodge theory for $L^{2}$-cohomology of infinite coverings. Topology, 16(2):157-165, 1977. MR0445560 (56:3898)

[11] Jozef Dódziuk, Peter Linnell, Varghese Mathai, Thomas Schick, and Stuart Yates. Approximating $L^{2}$-invariants, and the Atiyah conjecture. Comm. Pure Appl. Math., 56(7):839-873, 2003. MR1990479 (2004g:58040)

[12] David S. Dummit and Richard M. Foote. Abstract algebra. Prentice Hall Inc., Englewood Cliffs, NJ, 2nd. edition, 1994. MR1138725 (92k:00007)

[13] Michael Falk and Richard Randell. The lower central series of a fiber-type arrangement. Invent. Math., 82(1):77-88, 1985. MR808110(87c:32015b)

[14] Michael Falk and Richard Randell. Pure braid groups and products of free groups. In Braids (Santa Cruz, CA, 1986), pages 217-228. Amer. Math. Soc., Providence, RI, 1988. MR975081 (90d:20070)

[15] R. Fenn, M. T. Greene, D. Rolfsen, C. Rourke, and B. Wiest. Ordering the braid groups. Pacific J. Math., 191(1):49-74, 1999. MR1725462 (2000j:20064)

[16] Rostislav I. Grigorchuk, Peter Linnell, Thomas Schick, and Andrzej Żuk. On a question of Atiyah. C. R. Acad. Sci. Paris Sér. I Math., 331(9):663-668, 2000. MR 1797748 (2001m:57050)

[17] Michael Gromov. Kähler hyperbolicity and $L_{2}$-Hodge theory. J. Differential Geom., 33(1):263-292, 1991. MR1085144 (92a:58133)

[18] K. W. Gruenberg. Residual properties of infinite soluble groups. Proc. London Math. Soc. (3), 7:29-62, 1957. MR0087652(19:386a)

[19] P. Hall. Some sufficient conditions for a group to be nilpotent. Illinois J. Math., 2:787-801, 1958. MR0105441(21:4183)

[20] John Hempel. Residual finiteness for 3-manifolds. In Combinatorial group theory and topology (Alta, Utah, 1984), pages 379-396. Princeton Univ. Press, Princeton, NJ, 1987. MR895623 (89b:57002)

[21] K. A. Hirsch. On infinite soluble groups. II. Proc. London Math. Soc. (2), 44:336-344, 1938.

[22] Ian Hughes. Division rings of fractions for group rings. Comm. Pure Appl. Math., 23:181-188, 1970. MR0263934 (41:8533)

[23] Ian Hughes. Division rings of fractions for group rings. II. Comm. Pure Appl. Math., 25:127131, 1972. MR0292956 (45:2037)

[24] Stephen P. Humphries. Torsion-free quotients of braid groups. Internat. J. Algebra Comput., 11(3):363-373, 2001. MR1847185 (2002f:20054)

[25] Stefan Jackowski. A fixed-point theorem for p-group actions. Proc. Amer. Math. Soc., 102(1):205-208, 1988. MR915745 (89a:57052)

[26] William Jaco. Lectures on three-manifold topology. American Mathematical Society, Providence, R.I., 1980. MR565450 (81k:57009) 
[27] Nathan Jacobson. Basic algebra. II. W. H. Freeman and Company, New York, second edition, 1989. MR:1009787 (90m:00007)

[28] S. O. Kochman. Bordism, stable homotopy and Adams spectral sequences. American Mathematical Society, Providence, RI, 1996. MR1407034 (97i:55017)

[29] P. H. Kropholler, P. A. Linnell, and J. A. Moody. Applications of a new $K$-theoretic theorem to soluble group rings. Proc. Amer. Math. Soc., 104(3):675-684, 1988. MR964842 (89j:16016)

[30] Inga Kümpel, Peter Linnell, and Thomas Schick. Galois cohomology of completed link groups. in preparation.

[31] John P. Labute. Algèbres de Lie et pro-p-groupes définis par une seule relation. Invent. Math., 4:142-158, 1967. MR0218495 (36:1581)

[32] John P. Labute. On the descending central series of groups with a single defining relation. $J$. Algebra, 14:16-23, 1970. MR0251111 (40:4342)

[33] John P. Labute. The Lie algebra associated to the lower central series of a link group and Murasugi's conjecture. Proc. Amer. Math. Soc., 109(4):951-956, 1990. MR.1013973 (90k:20065)

[34] V. Lin. Braids, permutations, polynomials I. Preprint, Max Planck Institut für Mathematik, Bonn, 112 pages, 1996.

[35] Peter A. Linnell. Division rings and group von Neumann algebras. Forum Math., 5(6):561576, 1993. MR.1242889 (94h:20009)

[36] Peter A. Linnell. Analytic versions of the zero divisor conjecture. In Geometry and cohomology in group theory (Durham, 1994), pages 209-248. Cambridge Univ. Press, Cambridge, 1998. MR1709960 (2000g:20016)

[37] Wolfgang Lück. Transformation groups and algebraic $K$-theory, volume 1408 of Lecture Notes in Mathematics. Springer-Verlag, Berlin, 1989. Mathematica Gottingensis. MR1027600 (91g:57036)

[38] Wolfgang Lück. $L^{2}$-invariants of regular coverings of compact manifolds and CW-complexes. In Handbook of geometric topology, pages 735-817. North-Holland, Amsterdam, 2002. MR1886681 (2003a:58049)

[39] Wolfgang Lück, Thomas Schick, and Thomas Thielmann. Torsion and fibrations. J. Reine Angew. Math., 498:1-33, 1998. MR1629917 (99e:58203)

[40] R. C. Lyndon. Two notes on nilpotent groups. Proc. Amer. Math. Soc., 3:579-583, 1952. MR0049889 (14:242a)

[41] Wilhelm Magnus. Uber beziehungen zwischen höheren Kommutatoren. J. Reine Angew. Math., 177:105-115, 1937.

[42] C. D. Papakyriakopoulos. On Dehn's lemma and the asphericity of knots. Ann. of Math. (2), 66:1-26, 1957. MR0090053 (19:761a)

[43] Donald S. Passman. Infinite crossed products. Academic Press Inc., Boston, MA, 1989. MR.979094 (90g:16002)

[44] Holger Reich. Group von Neumann algebras and related algebras. Ph.D. thesis, Universität Göttingen, 1999. http://www.math.uni-muenster.de/u/lueck/publ/diplome/reich.dvi.

[45] Dale Rolfsen and Jun Zhu. Braids, orderings and zero divisors. J. Knot Theory Ramifications, 7(6):837-841, 1998. MR1643939 (99g:20072)

[46] Thomas Schick. Finite group extensions and the Baum-Connes conjecture. preprint, available via http://arXiv/math.KT/0209165.

[47] Thomas Schick. Integrality of $L^{2}$-Betti numbers. Math. Ann., 317(4):727-750, 2000. MR.1777117 (2002k:55009a)

[48] Thomas Schick. Erratum: "Integrality of $L^{2}$-Betti numbers". Math. Ann., 322(2):421-422, 2002. MR1894160 (2002k:55009b)

[49] Jean-Pierre Serre. Cohomologie Galoisienne. Springer-Verlag, Berlin, fifth edition, 1994. MR:1324577 (96b:12010)

[50] Jean-Pierre Serre. Galois cohomology. Springer-Verlag, Berlin, 1997. Translated from the French by Patrick Ion and revised by the author. MR.1466966 (98g:12007)

[51] John Stallings. Homology and central series of groups. J. Algebra, 2:170-181, 1965. MR0175956 (31:232)

[52] Robert M. Switzer. Algebraic topology - homotopy and homology. Springer-Verlag, New York, 1975. Die Grundlehren der mathematischen Wissenschaften, Band 212. MR0385836 (52:6695)

[53] John S. Wilson. Profinite groups. The Clarendon Press Oxford University Press, New York, 1998. MR.1691054 (2000j:20048) 
Department of Mathematics, Virginia Tech, Blacksburg, Virginia 24061-0123

E-mail address: linnell@math.vt.edu

URL: http://www.math.vt.edu/people/linnell/

Mathematisches Institut, Georg-August-Universität Göttingen, Bunsenstr. 3-5, 37073 Göttingen, Germany

E-mail address: schick@uni-math.gwdg.de

URL: http://www. uni-math.gwdg.de/schick/ 